\title{
Oxidation und epitaktische Oxidation von Nickel und Nickellegierungen
}

\author{
Dissertation \\ zur Erlangung des Doktorgrades \\ der Mathematisch-Naturwissenschaftlichen Fakultäten \\ der Georg-August-Universität zu Göttingen
}

vorgelegt von

\section{Cirsten Maja Brandt}

aus Salzgitter-Bad

Göttingen 2002 
D 7

Referent: $\quad$ Prof. Dr. H.C. Freyhardt

Korreferent: $\quad$ Prof. Dr. K. Winzer

Tag der mündlichen Prüfung : 16.8.2002 


\section{Inhaltsverzeichnis}

1 Einleitung............................................................................................................. 1

2 Experimentelle Methoden...........................................................................4

2.1 Röntgendiffraktometrie (XRD) 4

2.1.1 Das 2-Kreis-Diffraktometer 5

2.1.2 Das 4-Kreis-Diffraktometer 5

2.2 Charakterisierung der Oberflächenmorphologie und der Mikrostruktur 6

$\begin{array}{lll}\text { 2.2.1 Rasterelektronenmikroskopie (REM) } & 6\end{array}$

2.2.2 Rasterkraftmikroskopie (AFM) 6

2.2.3 Transmissionselektronenmikroskopie (TEM) 6

$\begin{array}{lll}2.2 .4 & \text { Profilometer } & 7\end{array}$

2.3 Herstellung der Proben $\quad 7$

2.3.1 Herstellung der polykristallinen $\mathrm{Ni}, \mathrm{Ni}_{56} \mathrm{Cu}_{44}$ und $\mathrm{Ni}_{84} \mathrm{Cr}_{16}$-Filme 7

2.3.2 Herstellung der IBAD-YSZ-Pufferschichten 8

2.3.3 Herstellung der RABiTS-Bänder 9

2.3.4 Herstellung der einkristallinen Nickel und $\mathrm{Ni}_{87} \mathrm{Cr}_{13}$-Filme 9

2.3.5 Oxidation der einkristallinen Nickel und $\mathrm{Ni}_{87} \mathrm{Cr}_{13}$-Filme $\quad 9$

3 Stabilität von Substrat-Puffer-Systemen................................................10

3.1 Simulationsexperimente zur thermischen Stabilität 11

3.2 Diskussion 16

3.2.1 Einfluss der thermischen Ausdehnung auf das Ablösen von Pufferschichten 16

3.2.2 Einfluss der Substratoxidation auf das Ablösen von Pufferschichten $\quad 18$

3.3 Schlussfolgerung $\quad 22$

4 Oxidation von RABiTS-Bändern unter hohen Temperaturen ............23

4.1 Die Phasenbildung $\quad 24$

4.2 Das epitaktische Wachstum 26

4.3 Der Volumenanteil der Orientierungen 29

4.4 Die Topologie der Oberflächen 31

4.5 Einfluss der Substratrauigkeit auf die Oxidrauigkeit 34

$\begin{array}{ll}4.6 & \text { Schlussfolgerung }\end{array}$ 
5.1 Charakterisierung der einkristallinen Filme 36

5.2 Vorzugsorientierungen in kompakten NiO-Filmen 38

5.2.1 Oxidation der einkristallinen Ni-Filme 38

5.2.2 Oxidation einkristalliner $\mathrm{Ni}_{87} \mathrm{Cr}_{13}$-Filme $\quad 39$

5.3 Texturentwicklung und Kinetik der Oxidation einkristalliner Nickelfilme 41

5.3.1 Texturentwicklung in dicken NiO-Schichten 41

5.3.2 Texturentwicklung in dünnen NiO-Schichten 44

5.4 Oberflächentopologie und Rauigkeit von NiO-Filmen 47

5.4.1 Die Frühstadien der Rauigkeitsentwicklung 47

5.4.2 Oberflächentopologie dicker NiO-Schichten 48

5.5 Oberflächen und Schichtaufbau der oxidierten einkristallinen NiCr-Filme 51

5.6 Löcherartige Strukturen in oxidierten einkristallinen Nickelfilmen $\quad 52$

5.7 Beschichtung YBaCuO 54

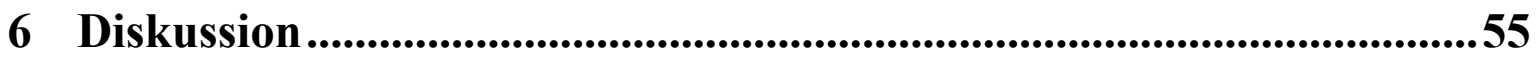

6.1 Oxidation von Metallen $\quad 55$

6.1.1 Oxidation von $\mathrm{Ni} 56$

6.1.2 Oxidation von $\mathrm{NiCr} \quad 57$

6.2 Kinetik der Oxidation von einkristallinen Nickelfilmen $\quad 57$

6.3 Texturentwicklung in epitaktisch oxidierten NiO-Schichten 60

$\begin{array}{lll}6.3 .1 & \text { Die NiO-Anfangsschicht } & 60\end{array}$

6.3.2 Die Umwandlungsreaktion 61

6.3.3 Modellvorstellung zur Texturentwicklung in epitaktisch oxidiertem NiO 64

6.3.4 Texturentwicklung im $\mathrm{NiO}$ bei der Oxidation von $\{100\}<100>\mathrm{Ni}_{87} \mathrm{Cr}_{13} \quad 66$

6.3.5 Einfluss weiterer Parameter auf die Oxidation 67

6.4 Entwicklung der Oberflächenmorphologie $\quad 68$

$\begin{array}{lll}\text { 6.4.1 Einfluss der Substratrauigkeit } & 68\end{array}$

6.4.2 Frühstadien der Rauigkeit 68

6.4.3 Entwicklung der Rauigkeit $\quad 69$

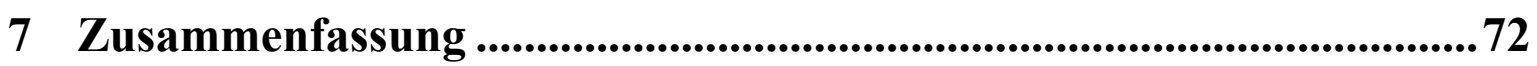

8 Literaturverzeichnis ................................................................................74

Danksagung........................................................................................................................78

Lebenslauf .............................................................................................................. 


\section{Einleitung}

Die Menschheit wird wahrscheinlich in diesem Jahrhundert mehr Energie benötigen als in ihrer gesamten bisherigen Geschichte [1]. Die wohl bedeutendste Entdeckung der letzten beiden Jahrhunderte, die Elektrizität, brachte unserer Gesellschaft einen sehr hohen Lebensstandard, führte aber gleichzeitig zu einer extremen Belastung des globalen Ökosystems und einer drastischen Rohstoffminimierung, so dass die Erforschung und Entwicklung alternativer Energiequellen und energiesparender Systeme in zunehmendem Maße an Bedeutung gewinnt. Bereits seit 1911 ist durch die Entdeckung der Supraleitung [2] bekannt, dass es theoretisch möglich ist, Energie verlustfrei zu transportieren und zu speichern. Aber erst 1986 mit der Entdeckung der Hochtemperatursupraleiter [3], bei denen die Sprungtemperatur über der des Siedepunkts vom Stickstoffs liegt, bekam dieses Phänomen eine neue technologische Qualität. Seitdem werden supraleitende Kabel und Bänder entwickelt z.B. für den Einsatz in supraleitenden Spulen und Strombegrenzern. Aber auch die magnetischen Eigenschaften der Supraleiter sind von großem Interesse, denn es können Remanenzen und Energiedichten erzeugt werden, die deutlich über denen liegen, die konventionell erreicht werden können. So finden konventionelle Supraleiter bereits heute ihren Einsatz in der Magnettomographie. Die magnetischen Eigenschaften sind besonders bei den supraleitenden Massivmaterialien von großem Interesse. Seit 1988 werden solche Materialien in ersten Prototypen eingesetzt, die das Prinzip der Levitation ausnutzen, so z.B. in Rotoren von Schwungmassenspeichern [4-6], in Transportsystemen und in sich selbst stabilisierenden magnetischen Lagern.

Hochtemperatursupraleiter sind perowskitische Oxide, die nicht nur in ihrer Kristallstruktur, sondern in allen elektrischen und supraleitenden Eigenschaften eine starke Anisotropie aufweisen. Das System Y-Ba-Cu-O [7] hat bereits nach kurzer Zeit aufgrund seiner relativ geringen Anisotropie, was einen nahezu verlustfreien Stromtransport auch in größeren äußeren Magnetfeldern ermöglicht, ein hohes Maß an Bedeutung gewonnen. Eine besondere Problematik dieses Systems stellt die kurze Kohärenzlänge der supraleitenden Ladungsträger da, so dass bereits Korngrenzen, die Verkippungswinkel von wenigen Grad aufweisen, die kritische Stromdichte erheblich reduzieren können [8]. Korngrenzen stellen in diesem Material also strukturell gestörte Zonen dar, die die supraleitenden Ladungsträger nur durch Tunnelprozesse passieren können. Um also gute supraleitende Eigenschaften zu er- 
zielen, müssen die Materialien eine nahezu einkristalline Textur aufweisen. Diese wird in Massivmaterialien durch modifizierte Einkristallzuchtverfahren z.B. die Schmelztexturierung $[9,10]$ erreicht. Die Herstellung von YBaCuO-Bandleitern, ein Analogon dünner Filme zu konventionellen Kabeln, geschieht durch das epitaktische Abscheiden mittels gepulster Laserdeposition (PLD) [11], Chemical Vapour Deposition (CVD) [12] oder Sputtern [13]. Als texturierte Unterlage wird ein Schichtsystem aus einem metallischem Substratband und einer oder mehrerer keramischer Pufferschichten verwendet. Die Pufferschichten dienen zum einen als Diffusionsbarriere zwischen Substrat und Supraleiter, da Interdiffusion die supraleitenden Eigenschaften zerstört, und müssen zum anderen aber auch einen guten Epitaxieübertrag auf den Supraleiter gewährleisten. Dieses Schichtsystem aus Substrat und Puffer wird momentan hauptsächlich nach zwei unterschiedliche Verfahren hergestellt, das IBAD-Verfahren [14] und das RABiTS-Verfahren [15].

Beim IBAD-Verfahren (Ion Beam Assisted Deposition) werden polykristalline technische Substrate wie z.B. Edelstähle, Nickel oder Nickellegierungen verwendet. Auf diese wird dann mittels Ionenstrahlsputterns Yttrium-stabilisiertes Zirkondioxid (YSZ) abgeschieden. Während dieser Deposition wird ein unterstützender Ionenstrahl unter einem Einschusswinkel von $55^{\circ}$ zur Substratnormalen auf den aufwachsenden YSZ-Film gerichtet. Die mittels IBAD hergestellten YSZ-Filme weisen eine biaxiale $\{100\}<100>$ Textur auf.

Beim RABiTS-Verfahren (Rolling Assisted Biaxially Textured Substrates) werden bereits die metallischen Substrate vortexturiert. Dabei werden metallische Bänder, hauptsächlich Nickel und Nickellegierungen, durch Walzprozesse so stark verformt, dass bei einem anschließenden Rekristallisationsglühen eine scharfe Würfeltextur entsteht. Auf diesen vortexturierten Substraten werden dann mittels epitaktischer Deposition die Pufferschichten abgeschieden, wobei zur Zeit hauptsächlich ein Schichtsystem aus $\mathrm{CeO}_{2}$ und YSZ verwendet wird. Mittels beider Verfahren lassen sich supraleitende Kurzproben herstellen, die hohe kritische Stromdichten von 2-3 MA/ $\mathrm{cm}^{2}$ bei $77 \mathrm{~K}$ im Nullfeld aufweisen. Bei der Herstellung längerer supraleitender Bänder werden weitaus spezifischere Anforderungen an das Substrat-Pufferschichtsystem gestellt. Das Substratband sollte zum einen eine gewisse mechanische Festigkeit aufweisen und zum anderen keine ferromagnetischen Eigenschaften besitzen. Beide Anforderungen können durch Legierungszusätze wie z.B. Kupfer, Chrom und Vanadium zu den reinen Nickelbändern erfüllt werden.

Bei der Herstellung von YBaCuO-Filmen, die mittels Laserdeposition abgeschieden werden, müssen hochreaktive Kondensationsbedingungen verwendet werden, denn die Temperatur, bei der $\mathrm{YBaCuO}$ epitaktisch und hochtexturiert auf der vortexturierten Unterlage aufwächst (Epitaxietemperatur), liegt bei ca. $750^{\circ} \mathrm{C}$, außerdem muss ein Sauerstoffpartialdruck von 0,6 mbar $\mathrm{O}_{2}$ eingestellt werden, damit sich die gewünschte $\mathrm{Y}_{1} \mathrm{Ba}_{2} \mathrm{Cu}_{3} \mathrm{O}_{7-\mathrm{x}^{-}}$ Phase in der orthorhombischen Struktur bildet. Diese hohen Kondensationsbedingungen 
führten bei der Herstellung längerer supraleitender Bänder häufig zu einem Ablösen der Pufferschichten vom Substrat. Daher wird im Kapitel 3 „Stabilität von Substrat-PufferSystemen“ zunächst dargestellt, welche Faktoren die thermische Stabilität eines Verbundes aus einem metallischen Subtstrat und einer Pufferschicht bestimmen.

Die epitaktische Abscheidung der Pufferschichten geschieht zur Zeit hauptsächlich noch mittels Sputterns und Elektronenstrahlverdampfens. Da diese Verfahren in Vakuumanlagen durchgeführt werden müssen, sind sie mit einem sehr hohen apparativen Aufwand verbunden. Mögliche Alternativen zu diesen Verfahren bieten chemische Depositionsverfahren wie das dip- bzw. das spin-coating. Eine weitere interessante Möglichkeit der Pufferschichtherstellung stellt die epitaktische Oxidation (SOE) (Surface Oxidation Epitaxy) $[16,17]$ dar. Bei diesem Verfahren werden vortexturierte Nickel- bzw. Nickellegierungssubstrate unter definierten Bedingungen gezielt oxidiert, so dass sich Nickeloxid epitaktisch auf der Substratunterlage bildet. Das Eigenoxid dient dann selber als Pufferschicht bzw. als Unterlage für weitere Pufferschichten. Im Kapitel 4 „Oxidation von RABiTS-Bändern unter hohen Temperaturen“ wird zunächst untersucht, inwieweit die auf Nickel- und Nickellegierungs-RABiTS-Bändern aufgewachsenen Eigenoxidschichten die für Pufferschichten erforderlichen Kriterien erfüllen.

Die Kinetik und die Mechanismen der Oxidation von Nickel und Nickellegierungen werden bereits seit 1952 [18] eingehend untersucht. Dagegen wurde die epitaktische Oxidation, d.h. die Oxidation von einkristallinen Ni(100)-Oberflächen bisher erst in ihren Anfangsstadien bis zur Ausbildung weniger Monolagen hauptsächlich mittels LEED (Low Energy Electron Diffraction) und RHEED (Reflexion High Energy Electron Diffraction) beobachtet [19-23]. Schwerpunkt und Ziel dieser Arbeit ist es deshalb, den Mechanismus und die Texturbildung in kompakten auf Ni(100)-Oberflächen oxidierten NiO-Schichten zu verstehen. Da RABiTS-Bänder eine ausgeprägte Mosaikverteilung hohe Verunreinigungen und Oberflächenrauigkeiten aufweisen, lässt sich der Einfluss einzelner Bandparameter auf das Oxidationsverhalten sehr schwer separieren. Deshalb wurden die Untersuchungen zum Oxidationsmechanismus anhand von Modellsystemen durchgeführt, wobei auf Einkristalle epitaktisch quasi-einkristalline Nickel- und Nickellegierungsschichten abgeschieden wurden. Im Kapitel 5 ,Epitakische Oxidation einkristalliner Ni und NiCr-Filme“" werden dann gezielte Untersuchungen an den einkristallinen Filmen durchgeführt, deren Ergebnisse dann die Basis für eine Diskussion der Mechanismen der Texturausbildung und der Rauigkeitsentwicklung bei der epitaktischen Oxidation bilden. 


\section{Experimentelle Methoden}

\subsection{Röntgendiffraktometrie (XRD)}

Die untersuchten Materialien und deren Texturen wurden in dieser Arbeit mittels Röntgenbeugung (XRD) charakterisiert. Bei der Röntgenbeugung wird unter einem Winkel $\Theta$ ein monochromatischer Röntgenstrahl auf die Probe gerichtet und die reflektierte Intensität von einem Detektor erfasst. Unter gewissen Winkeln interferiert der an den verschiedenen Netzebenen der Probe reflektierte Röntgenstrahl konstruktiv. Unter welchen Winkeln diese konstruktive Interferenz auftritt, wird durch die Bragg-Beziehung beschrieben $\mathrm{n} \lambda=2 \mathrm{~d}_{\mathrm{hkl}} \sin \theta$. Dabei ist $\lambda$ die Wellenlänge der monochromatischen Röntgenstrahlung, $\mathrm{d}_{\mathrm{hkl}}$ der Netzebenenabstand, (hkl) die Millerschen Indizes und $\Theta$ der Winkel des einfallenden bzw. reflektierten Röntgenstrahls.

Die (hkl)-orientierten Körner können nun nicht nur senkrecht zur Filmebene (out-ofplane), sondern auch in der Ebenen (in-plane) eine Vorzugsrichtung aufweisen. $\mathrm{Ob}$ ein Material eine Vorzugsrichtung zeigt, kann mit der Messung von Polfiguren nachgewiesen werden. Dazu wird eine bestimmte Reflexbedingung eingestellt, d.h. ein fester Einfallsund Ausfallswinkel $\Theta$, und die reflektierte Intensität in alle Raumrichtungen gemessen. Dazu wird die Probe um einen Winkel $\phi$ von $0^{\circ}-360^{\circ}$ gedreht und einen Winkel $\chi$ von $0^{\circ}$ $90^{\circ}$ gekippt. Zur Messung der (100)-Textur in einem kubisch flächenzentrierten Material kann z.B. auf dem (111)-Reflex gemesssen werden. Unter einem $\chi$-Winkel von $54,7^{\circ}$ werden dann bei einer in-plane Vorzugsorientierung 4 Reflexe beobachtet, in einem Abstand bei dem der $\phi$-Winkel $90^{\circ}$ beträgt. Zur schnelleren Bestimmung der in-plane Textur reicht es aus, lediglich einen $\phi$-Scan aufzunehmen. Dabei wird die Probe um einen $\chi$-Winkel von $54,7^{\circ}$ gekippt und einmal um einen $\phi$-Winkel von $360^{\circ}$ um sich selbst gedreht. Die Polfigur des (111) Reflexes eines kubischen (100)-texturierten Materials ist in Abb. 2.1a schematisch dargestellt. Die Abbildung 2.1b zeigt den zugehörigen $\phi$-Scan bei einem Verkippungswinkel von $\chi=54,7^{\circ}$. Die Güte der Textur wird durch die Halbwertsbreite (Full Width at Half Maximum (FWHM)) der Reflexe in der Polfigur bestimmt. Die Halbwertsbreiten der Reflexe in $\chi$-Richtung bestimmen die Güte der out-of-plane Textur und die Halbwertsbreiten in $\phi$-Richtung die der in-plane Textur. 
a)

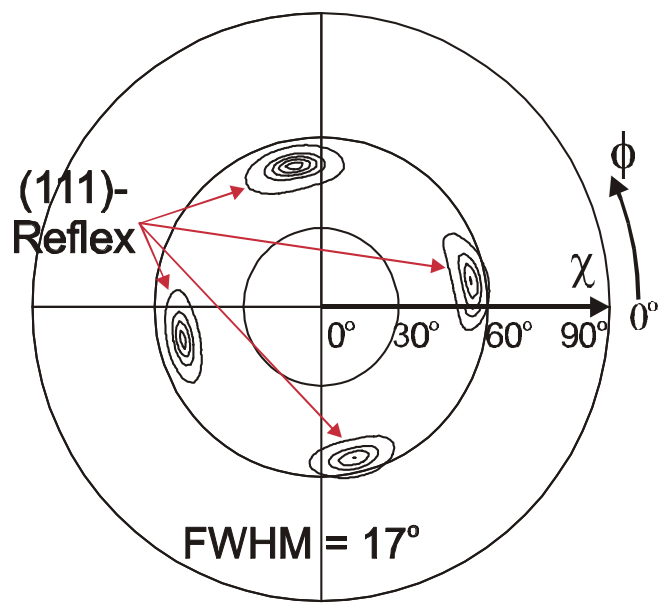

b)

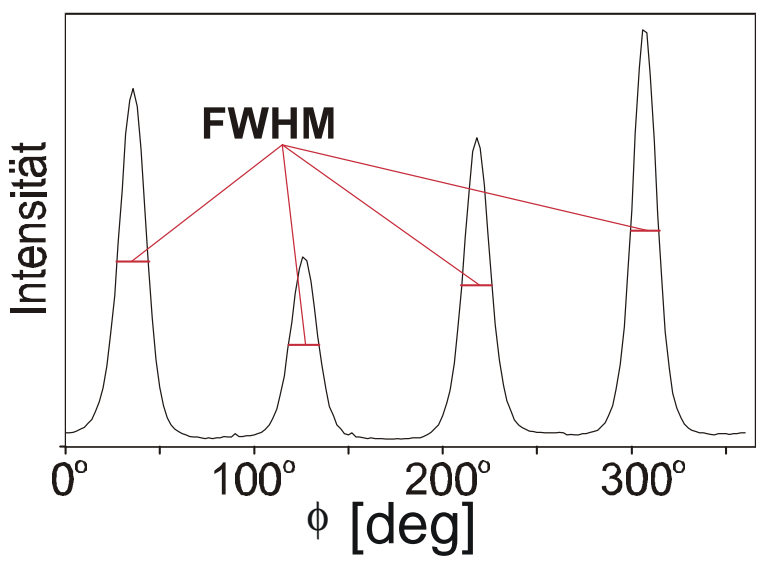

Abb. 2.1 a) Polfigur eines (100)-texturierten kubischen Materials (YSZ) gemessen auf dem (111) Reflex b) $\phi$-Scan aus der in 1a gezeigten Polfigur mit einem Verkippungswinkel von $\chi=54,7^{\circ}$.

\subsubsection{Das 2-Kreis-Diffraktometer}

Die Messungen der $\Theta-2 \Theta$ Diffraktogramme und der Rockingkurven $(\chi$-Scan) wurden in einem 2-Kreis-Diffraktometer D500 (Fa. Siemens) mit Bragg-Brentano-Geometrie durchgeführt (Co-K $\alpha_{\alpha}$-Strahlung: $\lambda=1,7903 \AA$ ). In dem 2 Kreis-Diffraktometer befindet sich die Probe in einem Rezipienten, der mittels Vorpumpe und Turbopumpe bis auf einen Partialdruck von unter $1 \cdot 10^{-5}$ mbar evakuiert werden kann. Weiterhin kann die Probe bis auf eine Temperatur von $680^{\circ} \mathrm{C}$ geheizt werden. Eine Gaszuleitung ermöglicht den Einlass von Sauerstoff. Somit ist es möglich, in-situ Oxidations- und in-situ Heizmessungen durchzuführen. Das 2-Kreis-Diffraktometer wird ausführlich in der Dissertation von H.U. Krebs [24] beschrieben.

\subsubsection{Das 4-Kreis-Diffraktometer}

Die Polfiguren und die $\phi$-Scans wurden in einem Texturgoniometer (Fa. Philips) des Mineralogisch-Kristallographischen Institut der Universität Göttingen und in einem Texturgoniometer D8 Advance (Fa. Bruker) des Zentrums für Funktionswerkstoffe gGmbH Göttingen durchgeführt. Verwendet wurde $\mathrm{Cu}-\mathrm{K}_{\alpha}$-Strahlung mit der Wellenlänge 1,54 ̊ bzw. Co-K $\mathrm{K}_{\alpha}$-Strahlung mit einer Wellenlänge von 1,7903 A. Das D8 Advance wird ausführlich in der Dissertation von J. Dzick [25] und das Gerät von der Fa. Philips wird in der Dissertation von J. Wiesman [26] beschrieben. 


\subsection{Charakterisierung der Oberflächenmorphologie und der Mikrostruktur}

\subsubsection{Rasterelektronenmikroskopie (REM)}

Die Oberflächenmorphologie der Schichten wird mit einen Rasterelektronenmikroskop (REM) Stereoscan 360 der Fa. Cambridge untersucht. Die Analyse der chemischen Elemente auf der Probenoberfläche geschieht mittels energiedispersiver Röntgenanalyse (EDX)( AN 10000 der Fa. Link). Mit dem EDX können sowohl qualitative als auch quantitative Elementanalysen durchgeführt werden. Außerdem können Elementverteilungsbilder aufgenommen werden.

\subsubsection{Rasterkraftmikroskopie (AFM)}

Die Oberflächenstrukturen der NiO-Schichten wurden weiterhin von C. Herweg mit einem Rasterkraftmikroskop (AFM = Atomic Force Microscope) NanoScopeIII MultiMode der Fa. Digital Instruments im Fachbereich Physiktechnik, Messtechnik und Feinwerktechnik der Fachhochschule Hildesheim/Holzminden/Göttingen vermessen. Dabei wird mit einer feinen Standardmessspitze aus geätztem Silizium (Spitzenradius <10nm) im TappingMode, gerastert. Dabei schwingt die Spitze vertikal mit einer Frequenz von 50.000500.000 Zyklen pro Sekunde. Durch zeitweiligen Kontakt der Spitze mit der Probenoberfläche wird die Amplitude der schwingenden Spitze abgeschwächt. Die örtliche Variation der Amplitude beschreibt somit die Oberflächenstruktur der Probe.

\subsubsection{Transmissionselektronenmikroskopie (TEM)}

Die transmissionselektronenmikroskopischen Aufnahmen der oxidierten Ni-Filme wurden von L.O. Kautschor an einem TEM der Fa. Philips (420ST EM) mit einer Beschleunigungsspannung von $120 \mathrm{kV}$ angefertigt. Für die Querschnittspräparation wird die Probe zuerst halbiert und die Oberflächen werden aufeinandergeklebt. Deshalb wurde zum Schutz und zur Markierung der Oberfläche auf den oxidierten Nickelfilm eine Goldschicht abgeschieden. Aus der zusammengeklebten Probe wird ein dünner Streifen geschnitten und in ein Metallplättchen eingebettet. Davon wird wiederum eine dünne Scheibe abgesägt. Diese Scheibe wird nun mit unterschiedlichen Schleifgeräten auf eine Dicke von 20-30 $\mu \mathrm{m}$ heruntergeschliffen. Schließlich wird die Probe mit einer Ionenstrahl-Dünnanlage (Duo Mill 600, Fa. Gatan) bis zur Durchstrahlbarkeit gedünnt. Eine genauere Beschreibung des 
TEMs und der Probenpräparation ist in der Dissertation von L.O. Kautschor [27] dargestellt.

\subsubsection{Profilometer}

Die Rauigkeiten der Substrate und der oxidierten Schichten wurden mit einem Mikroabtastsystem (Profilometer, Fa. Feinprüf-Perthen) bestimmt. Dabei wird mit einer kleinen Nadel (Diamantspitze $(\mathrm{r}=5 \mu \mathrm{m})$ ) über die Oberfläche der Probe gefahren und die Auslenkung der Nadel wird elektronisch verarbeitet und als Oberflächenprofil ausgegeben, wobei eine Auflösung von bis $10 \mathrm{~nm}$ erreicht wird. Das Gerät misst verschiedene Rauigkeitswerte, von denen die wichtigsten im folgenden kurz erklärt werden. Der Mittenrauwert $R_{a}$ ist der arithmetische Mittelwert der Beiträge aller Profilwerte des Rauheitprofils $\left(\mathrm{R}_{\mathrm{a}}=1 / \mathrm{N} \sum\left|\mathrm{Y}_{\mathrm{j}}\right|\right)$ und der Mittenrauwert $\mathrm{R}_{\mathrm{q}}$ (oder auch $\mathrm{R}_{\mathrm{MS}}$ genannt) ist der quadratische Mittelwert aller Profilwerte des Rauheitprofils $\left(R_{q}=\left(\sum Y_{j}^{2} / N\right)^{1 / 2}\right)$. Beide Werte sind in der Abbildung 2.2a schematisch dargestellt. Der $R_{\mathrm{z}}$ Wert ist die gemittelte Rautiefe, d.h. der Mittelwert aus aufeinanderfolgende Einzelrautiefen, wobei die Einzelrautiefe der senkrechte Abstand des höchsten vom tiefsten Profilpunkt ist. Zur besseren Vorstellung ist die Messung des $\mathrm{R}_{\mathrm{Z}}$-Wertes in Abbildung 2.2b dargestellt. Der $\mathrm{R}_{\mathrm{Z}}$ Wert ist hier der Mittelwert aus $Z_{1}-Z_{5}$.

a)

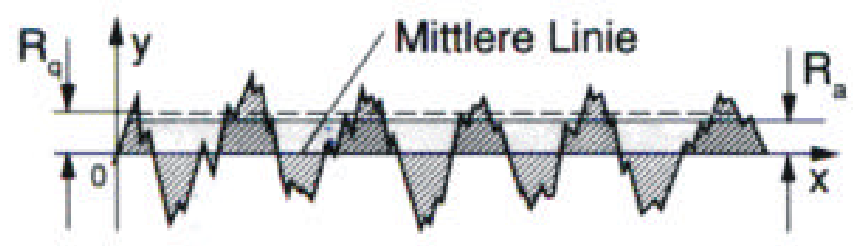

b)

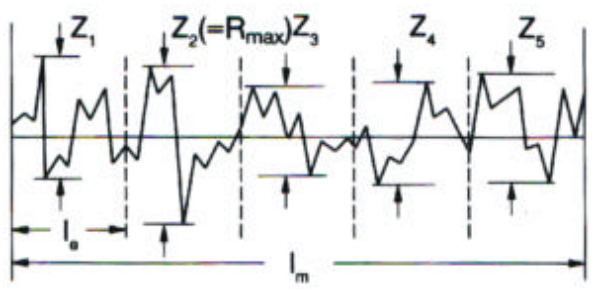

Abb. 2.2 a) schematische Darstellung der Mittenrauwerte $R_{a}$ und $R_{q}$ b) schematische Darstellung der gemittelten Rautiefe $\mathbf{R}_{\mathbf{r}}$.

\subsection{Herstellung der Proben}

\subsubsection{Herstellung der polykristallinen $\mathrm{Ni}, \mathrm{Ni}_{56} \mathrm{Cu}_{44}$ und $\mathrm{Ni}_{84} \mathrm{Cr}_{16}-\mathrm{Filme}$}

Die polykristallinen Filme aus Nickel und Nickellegierungen sind in einer UHV-Anlage mittels Magnetronsputterns deponiert wurden. In der Anlage befinden sich zwei TriodenMagnetron-Sputterquellen, die beide mit Argon betrieben werden. Diese Quellen sind so konstruiert, dass sich ein Plasma über dem Target bildet. Wird eine Spannung an das Tar- 
get angelegt, so schlagen die Ionen des Plasmas Atome aus dem Targetmaterial heraus. Die Nickellegierungen wurden hergestellt, indem eine Quelle mit einem Nickeltarget und die andere mit einem Kupfer- bzw. Chromtarget betrieben wurde. Eine genaue Beschreibung der Magnetronsputteranlage befindet sich in der Diplomarbeit von J. Hoffmann [28].

\subsubsection{Herstellung der IBAD-YSZ-Pufferschichten}

Die YSZ-Schichten werden in einer Hochvakuumapparatur mittels reaktiven Ionenstrahlsputterns hergestellt. In Abbildung 2.3 ist der schematische Aufbau der Anlage dargestellt.

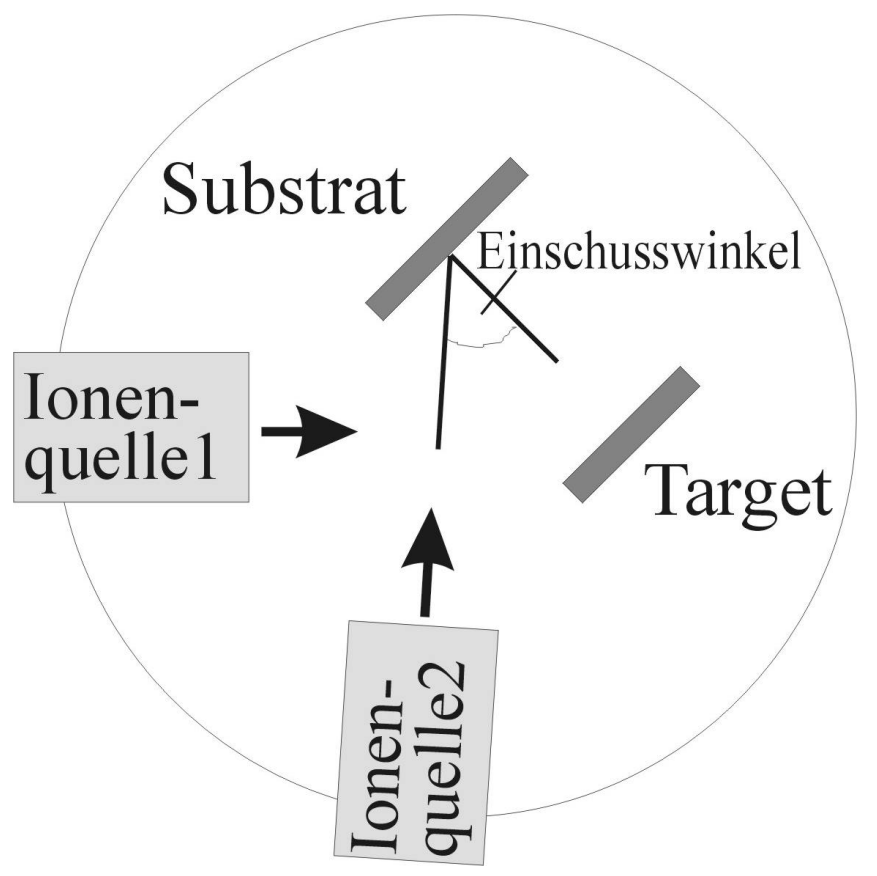

Abb. 2.3 Schematische Zeichnung der Ionenstrahl-Sputteranlage.

Die Sputterquellen sind Kaufmann-Ionenquellen (Fa. Iontech) mit einem Strahldurchmesser von je $2,5 \mathrm{~cm}$. Sie werden ausführlich von H.R. Kaufmann beschrieben [29]. Die Quelle 1 ist auf das Target gerichtet und wird mit Xenon betrieben. Die mit $1000 \mathrm{~V}$ beschleunigten Ionen treffen auf das Target und tragen dort das Targetmaterial ab, welches dann auf dem Substrat deponiert wird. Die zweite Ionenquelle ist unter einem bestimmten Winkel zur Substratnormalen auf das Substrat gerichtet und wird mit Argon betrieben. Mit dieser werden die Substrate vor Beginn des Depositionsprozesses von Adsorbaten an der Oberfläche gereinigt. Außerdem wird diese Quelle für die ionenstrahlunterstützende Deposition (IBAD) verwendet. Mittels dieses zusätzlichen Ionenbeschusses lassen sich bestimmte Materialien biaxial texturieren [14]. In dieser Arbeit werden die verwendeten YSZ-Filme mit diesem Verfahren biaxial texturiert. Eine genauere Charakterisierung der 
IBAD-texturierten YSZ-Filme befindet sich im Kapitel 3. Eine genaue Untersuchung zum Mechanismus der biaxialen Texturierung von YSZ mittels ionenstrahlunterstützter Deposition wird von J. Dzick [25] in seiner Dissertation beschrieben. Eine ausführliche Beschreibung der Ionenstrahlsputteranlage liefert J. Wiesmann [30].

\subsubsection{Herstellung der RABiTS-Bänder}

Das RABiTS-Verfahren (Rolling Assisted Biaxially Textured Substrates) wurde zur Vortexturierung von Substraten von A. Goyal et al. [15] entwickelt. Dabei werden metallische Substrate durch Walzprozesse zu über 90\% kaltverformt. Bei einem anschließenden Rekristallisationsglühen bei Temperaturen von über $1000^{\circ} \mathrm{C}$ bilden sich scharfe Würfeltexturen aus. Die in dieser Arbeit verwendeten NiCr und NiV-RABiTS Bänder wurden im Instiut für Werkstoffkunde in Dresden hergestellt. Die Ni-Bänder wurden vom Northwest Institute for Nonferrous Metals in Xi'an in China zur Verfügung gestellt.

\subsubsection{Herstellung der einkristallinen Nickel und $\mathrm{Ni}_{87} \mathrm{Cr}_{13}$-Filme}

Die einkristallinen Filme werden ebenfalls in der eben beschriebenen Ionenstrahlsputteranlage hergestellt. Als Substrate werden $\mathrm{SrTiO}_{3}(100) \mathrm{K}$-Einkristalle von der Firma CrysTec verwendet. Die Einkristalle werden auf einem heizbaren Substrathalter auf eine Temperatur von $300^{\circ} \mathrm{C}$ erhitzt. Bei dieser Temperatur wachsen die $\mathrm{Ni}$ und $\mathrm{Ni}_{87} \mathrm{Cr}_{13}$-Filme epitaktisch auf dem $\mathrm{SrTiO}_{3}$-Einkristall auf. Eine genaue Charakterisierung der verwendeten einkristallinen Filme wird in Kapitel 5 dargestellt.

\subsubsection{Oxidation der einkristallinen Nickel und $\mathrm{Ni}_{87} \mathrm{Cr}_{13}$-Filme}

Die ex-situ Oxidationsexperimente werden ebenfalls in der Ionenstrahlsputteranlage durchgeführt. Dazu wird die Anlage zunächst bis auf einen Restgasdruck von $6 \cdot 10^{-6}$ mbar evakuiert, dann wird der einkristalline Film auf eine bestimmte Temperatur erhitzt und schließlich wird ein definierter Sauerstoffpartialdruck eingelassen. Während der Abkühlphase ist die Anlage wieder evakuiert. Testweise wurden Nickelfilme vor der Oxidation mit dem Ionenstrahl beschossen, um eine eventuell bestehende natürliche Oxidschicht zu entfernen. Da der Ionenstrahlbeschuss aber keinen Einfluss auf die Textur der NiO-Schicht hatte, wurden die Oxidationsexperimente ohne vorheriges Ionenstrahlätzen durchgeführt. 


\section{Stabilität von Substrat-Puffer-Systemen}

Bei der Herstellung von YBaCuO-Filmen, die mittels Laserdeposition abgeschieden werden, müssen hochreaktive Kondensationsbedingungen verwendet werden: die Temperatur, bei der $\mathrm{YBaCuO}$ epitaktisch und hochtexturiert auf der vortexturierten Unterlage aufwächst (Epitaxietemperatur), liegt bei ca. $750^{\circ} \mathrm{C}$, außerdem muss ein Sauerstoffpartialdruck von 0,6 mbar $\mathrm{O}_{2}$ eingestellt werden, damit sich die gewünschte $\mathrm{Y}_{1} \mathrm{Ba}_{2} \mathrm{Cu}_{3} \mathrm{O}_{7-\mathrm{x}^{-}}$ Phase in der orthorhombischen Struktur bildet. Bei der Herstellung von Kurzproben stellen diese hochreaktiven Bedingungen in der Regel kein Problem dar. Bei der Entwicklung kontinuierlicher Herstellungsprozesse, bei welchen zunächst längere Bänder beschichtet wurden, war ein zentrales Problem die häufige Ablösung der Pufferschicht vom Substrat während der $\mathrm{YBaCuO}$-Deposition aufgrund der prozessbedingten längeren Verweildauer der einzelnen Substratabschnitte im reaktiven Beschichtungsmilieu. Diese Schädigungen sind zum Teil zwar nur lokal, trotzdem bewirken die gestörten Bereiche eine erhebliche Verringerung der Sprungtemperatur $\mathrm{T}_{\mathrm{c}}$ und der kritischen Stromdichte $\mathrm{j}_{\mathrm{c}}$. Deshalb ist es gerade für den kontinuierlichen Herstellungsprozess wichtig, eine geeignete Kombination aus Substrat- und Puffermaterial zu verwenden. Als Substratmaterialien haben sich bisher Ni-Basislegierungen herauskristallisiert, da sie genau wie das Nickel mittels RABiTSVerfahren texturiert werden können, der Zusatz aber den Ferromagnetismus des Nickels unterdrückt und zusätzlich das Material mechanisch härtet. Als Puffermaterialien werden meist keramische Oxide wie $\mathrm{YSZ}, \mathrm{CeO}_{2}$ oder $\mathrm{CGdO}$ verwendet. Die Pufferschicht dient zum einen als Diffusionsbarriere zwischen dem metallischen Substrat und dem keramischen Supraleiter, da die Diffusion von Metallkationen in den Supraleiter die supraleitenden Eigenschaften des $\mathrm{YBaCuO}$ zerstört, muss aber zum anderen auch den Epitaxieübertrag vom Substratband auf das $\mathrm{YBaCuO}$ gewährleisten. Das Ablösen des Puffers vom Substrat während der YBaCuO-Deposition kann nun mehrere Ursachen haben. Die unterschiedlichen thermischen Ausdehnungen von Substrat und Puffer verursachen beim Aufheizen auf die YBaCuO-Beschichtungstemperatur Spannungen, die ein Abplatzen der Pufferschicht bewirken könnten. Da die verwendeten keramischen Oxide gute Sauerstoffionenleiter sind, ist es ebenso möglich, dass eine Oxidbildung an der Grenzfläche zwischen Substrat und Puffer deren Ablösung hervorruft. Spielt die Substratoxidation eine wesentliche Rolle bei der Ablösung der Filme, so stellt sich die Frage, inwieweit die Eigenschaf- 
ten der Pufferschichten z.B. Textur, Dicke oder gestörtes Filmwachstum bzw. die chemische Zusammensetzung des Substrats die Oxidation minimieren können.

\subsection{Simulationsexperimente zur thermischen Stabilität}

Um zu simulieren, wie sich verschiedene Ni-Basislegierungen mit unterschiedlich texturierten Pufferschichten während der $\mathrm{YBaCuO}$-Deposition verhalten, werden in diesem Kapitel $\mathrm{Ni}, \mathrm{Ni}_{56} \mathrm{Cu}_{44}$, und $\mathrm{Ni}_{84} \mathrm{Cr}_{16}$ mit unterschiedlich texturierten und dicken IBAD-YSZSchichten bei Temperaturen von $200^{\circ} \mathrm{C}$ bis $700^{\circ} \mathrm{C}$ im Hochvakuum $\left(5 \cdot 10^{-6} \mathrm{mbar} \mathrm{O}_{2}\right)$ und

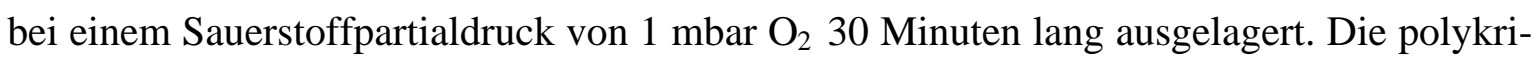
stallinen Ni- und Ni-Legierungsschichten wurden mittels Magnetronsputterns auf Saphirsubstrate abgeschieden und haben eine Schichtdicke von ungefähr $500 \mathrm{~nm}$. Die so hergestellten Modellsubstrate wurden mit jeweils einer dicken gut texturierten IBAD-YSZSchicht (Dicke: $800 \mathrm{~nm}$, out-of-plane-Textur: $7^{\circ}$ FWHM, in-plane-Textur: 20 ${ }^{\circ} \mathrm{FWHM}$ ) und einer dünnen weniger gut texturierten IBAD-YSZ-Schicht (Dicke: $300 \mathrm{~nm}$, out-ofplane-Textur: $9^{\circ}$ FWHM, in-plane-Textur: $35^{\circ} \mathrm{FWHM}$ ) beschichtet.

Die Modellschichtsysteme wurden nach jedem Auslagerungsschritt röntgenographisch, lichtmikroskopisch, mittels Rasterelektronenmikroskopie (REM) und mittels EDX untersucht.

Keine der oben genannten Untersuchungsmethoden zeigte eine Veränderung der nach den Auslagerungsschritten bis zu $700^{\circ} \mathrm{C}$ im Hochvakuum. Unabhängig von der Legierungszusammensetzung sind die Modellschichtsysteme hinsichtlich der Temperaturbehandlung stabil. Die unterschiedlichen thermischen Ausdehnungen spielen also für sich genommen keine Rolle bei der Ablösung der Schichten.

In Abb 3.1 sind die $\Theta-2 \Theta$ Röntgendiffraktogramme der einzelnen Schichten nach einer 30minütigen Auslagerung bei $700^{\circ} \mathrm{C}$ und $1 \mathrm{mbar}^{\mathrm{O}_{2}}$ dargestellt. Es tritt hier, anders als bei den im Vakuum ausgelagerten Schichten, z.T. eine Oxidbildung auf. Die Röntgengeometrie und Röntgenzeiten waren bei allen Messungen die gleichen, so dass unterschiedliche Röntgenintensitäten ein Maß für unterschiedliche Oxidanteile darstellen. Das NiCu-System zeigt die stärksten Oxidreflexe. Der NiO(111)-Reflex hat eine Intensität von 3000 Zählereignissen pro Sekunde. Sehr deutlich sind auch unterschiedliche CuO-Reflexe zu erkennen. Auch die Modellschichtsysteme mit dem reinen Ni-Film als Substrat zeigen NiOReflexe. Die Intensität des NiO(111)-Reflexes beträgt hier unabhängig von der Dicke und der Textur der YSZ-Pufferschicht ungefähr 250 Zählereignisse pro Sekunde. Die Modellschichtsystemen mit den NiCr-Filmen als Substrat zeigen keine Oxidreflexe. 


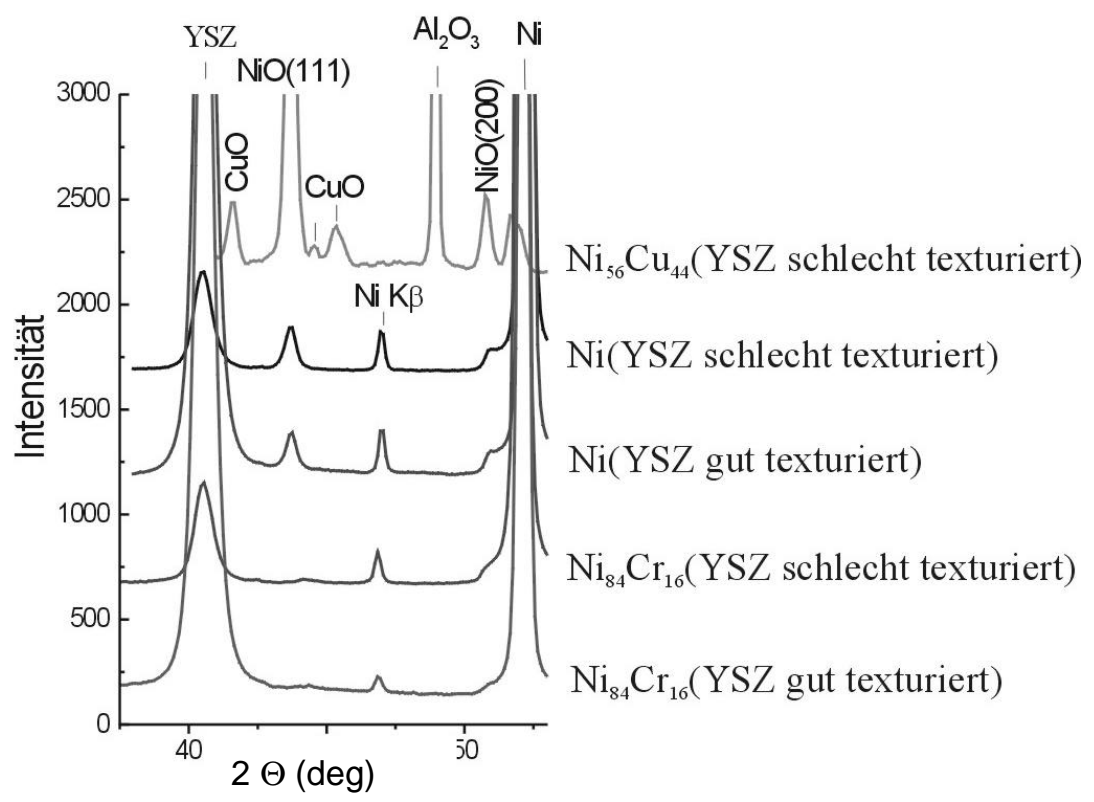

Abb 3.1 $\Theta-2 \Theta$ Röntgendiffraktogramme der bei $700^{\circ} \mathrm{C}$ und $1 \mathrm{mbar} \mathrm{O}_{2}$ ausgelagerten Modellschichtsysteme.

In Abb. 3.2a-c sind REM-Aufnahmen des NiCu-YSZ-Schichtsystems nach einer Oxidation bei $500^{\circ} \mathrm{C}, 600^{\circ} \mathrm{C}$ und $700^{\circ} \mathrm{C}$ unter 1 mbar $\mathrm{O}_{2}$ abgebildet.

a)

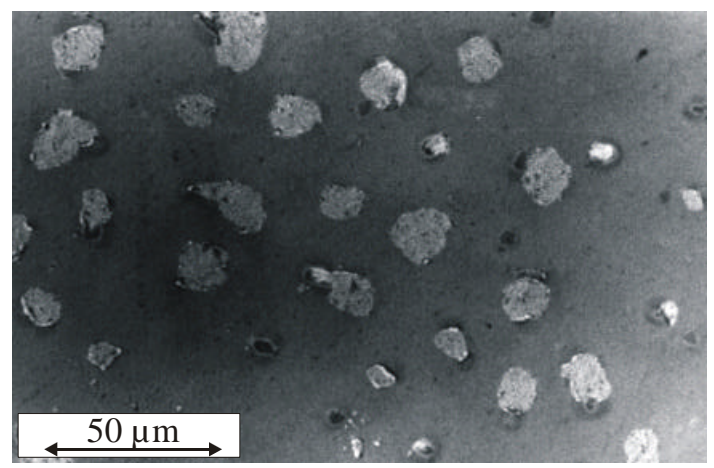

c) b)

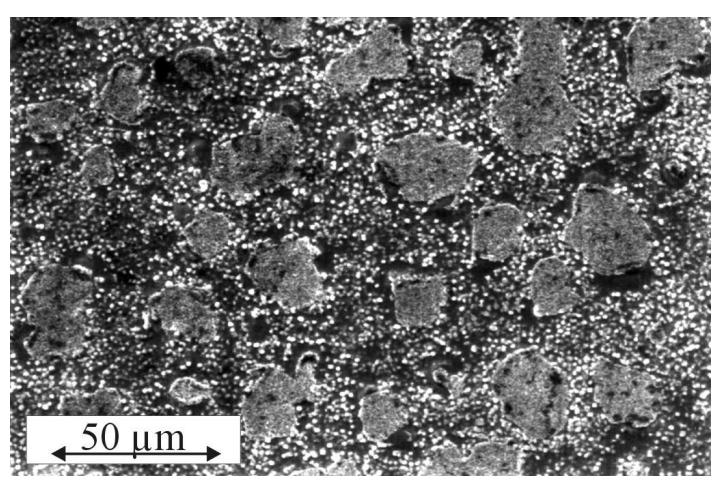

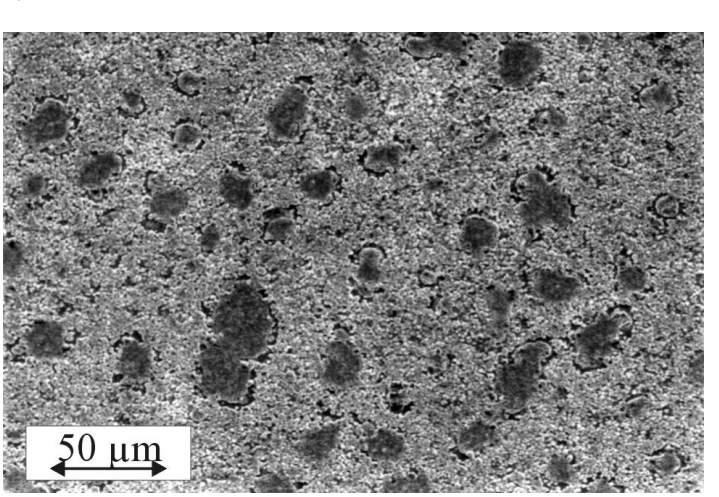

Abb. 3.2 REM-Aufnahmen einer zuerst bei a) $500^{\circ} \mathrm{C}$, dann bei b) $600^{\circ} \mathrm{C}$ und schließlich bei c) $700^{\circ} \mathrm{C}$ jeweils bei 30 min lang oxidierten $\mathrm{NiCu}$-YSZ-Schicht. Der Sauerstoffpartialdruck betrug 1 mbar $\mathrm{O}_{2}$. 
Bereits bei einer Auslagerungstemperatur von $500^{\circ} \mathrm{C}$ sind viele Bereiche des YSZ-Films abgeplatzt (Abb. 3.2a), die abgeplatzten Stellen haben im Mittel einen Durchmesser von $13 \mu \mathrm{m}$. Elementverteilungsbilder, die mit dem EDX aufgenommen wurden, zeigen, dass an den abgeplatzten Bereichen eine sehr hohe Konzentration der Elemente Kupfer und Sauerstoff vorliegen, während die Intensität des Elements Nickel sehr gering ist. In den Bereichen zwischen den abgeplatzten Stellen ist der YSZ-Film optisch unversehrt. Abbildung $3.2 \mathrm{~b}$ zeigt denselben Film nach einer weiteren 30 minütigen Auslagerung bei $600^{\circ} \mathrm{C}$ und 1 mbar $\mathrm{O}_{2}$. Deutlich erkennbar ist, dass sich die schon vorher abgeplatzten Bereiche vergrößert haben, der mittlere Durchmesser beträgt nun $27 \mu \mathrm{m}$. Außerdem ist zu erkennen, dass sich zwischen den großen abgeplatzten Bereichen viele sehr kleine Bereiche befinden, die sich ablösen. Abbildung 3.2c zeigt denselben Film nach einer weiteren Oxidation bei $700^{\circ} \mathrm{C}$.

a)

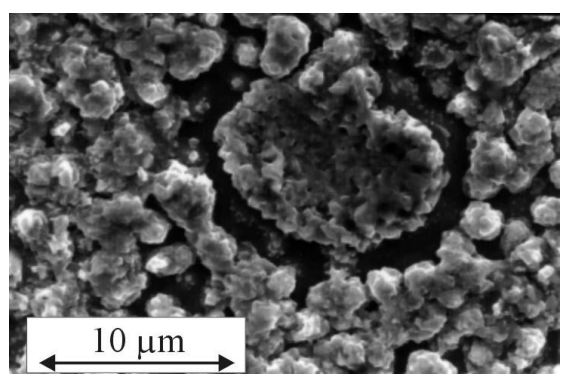

b)

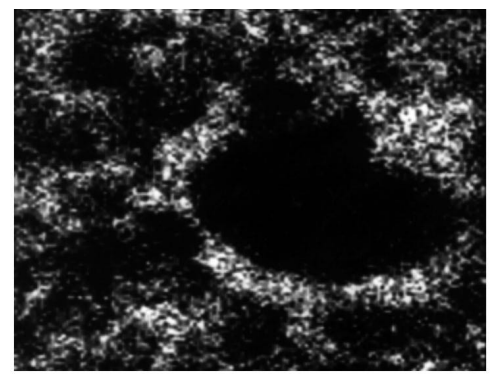

Zr-Verteilung

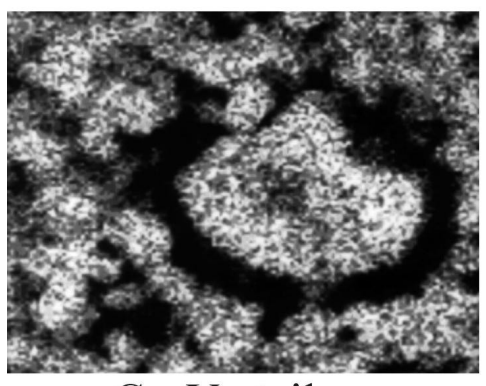

$\mathrm{Cu}$-Verteilung c)

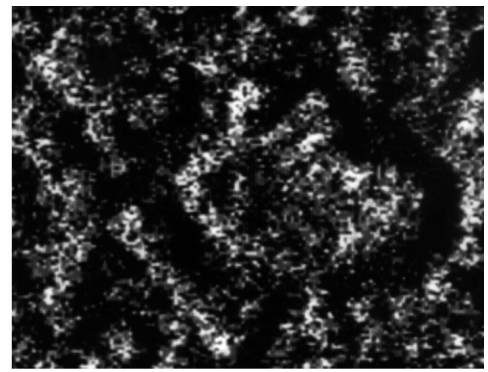

O-Verteilung

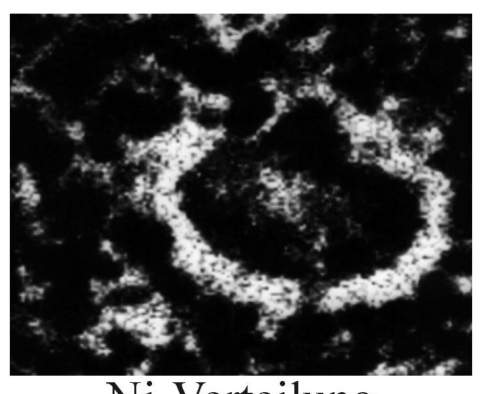

Ni-Verteilung

e)

Abb 3.3 a-e REM-Aufnahme einer bei $700^{\circ} \mathrm{C}$ oxidierten NiCu-YSZ-Schicht und die zugehörigen Elementverteilungsbilder der Elemente Zirkon, Sauerstoff, Kupfer und Nickel. 
Die großen abgeplatzten Bereiche sind auf einen mittleren Durchmesser von $32 \mu \mathrm{m}$ angewachsen und die dazwischenliegenden kleineren abgeplatzten Bereiche haben sich stark vermehrt und sind teilweise zusammengewachsen. In den Abbildung 3.3a-e ist eine weitere REM-Aufnahme des Filmes mit den zugehörigen Elementverteilungsbildern der Elemente Zirkon, Sauerstoff, Kupfer und Nickel dargestellt. Wie das Elementverteilungsbild der Zirkonverteilung zeigt, ist die YSZ-Schicht weitgehend abgeplatzt. An diesen Bereichen zeigt das Elementverteilungsbild des Kupfers und des Sauerstoffs hohe und das Elementverteilungsbild des Nickels geringe Intensitäten an. Dies läßt darauf schließen, dass sich an der Grenzfläche zum YSZ CuO gebildet hat, das letztendlich dessen Abriss zur Folge hat.

Die Abbildungen 3.4a,b zeigen lichtmikroskopische Aufnahmen einer Ni-YSZ-Schicht nach 30 minütiger Auslagerung bei $600^{\circ} \mathrm{C}$ und $1 \mathrm{mbar} \mathrm{O}_{2}$. Da die REM-Aufnahmen eine unversehrte Oberfläche des YSZ-Films zeigen, befinden sich die lichtmikroskopisch sichtbaren Strukturen an der Grenzfläche Ni-YSZ.

a)
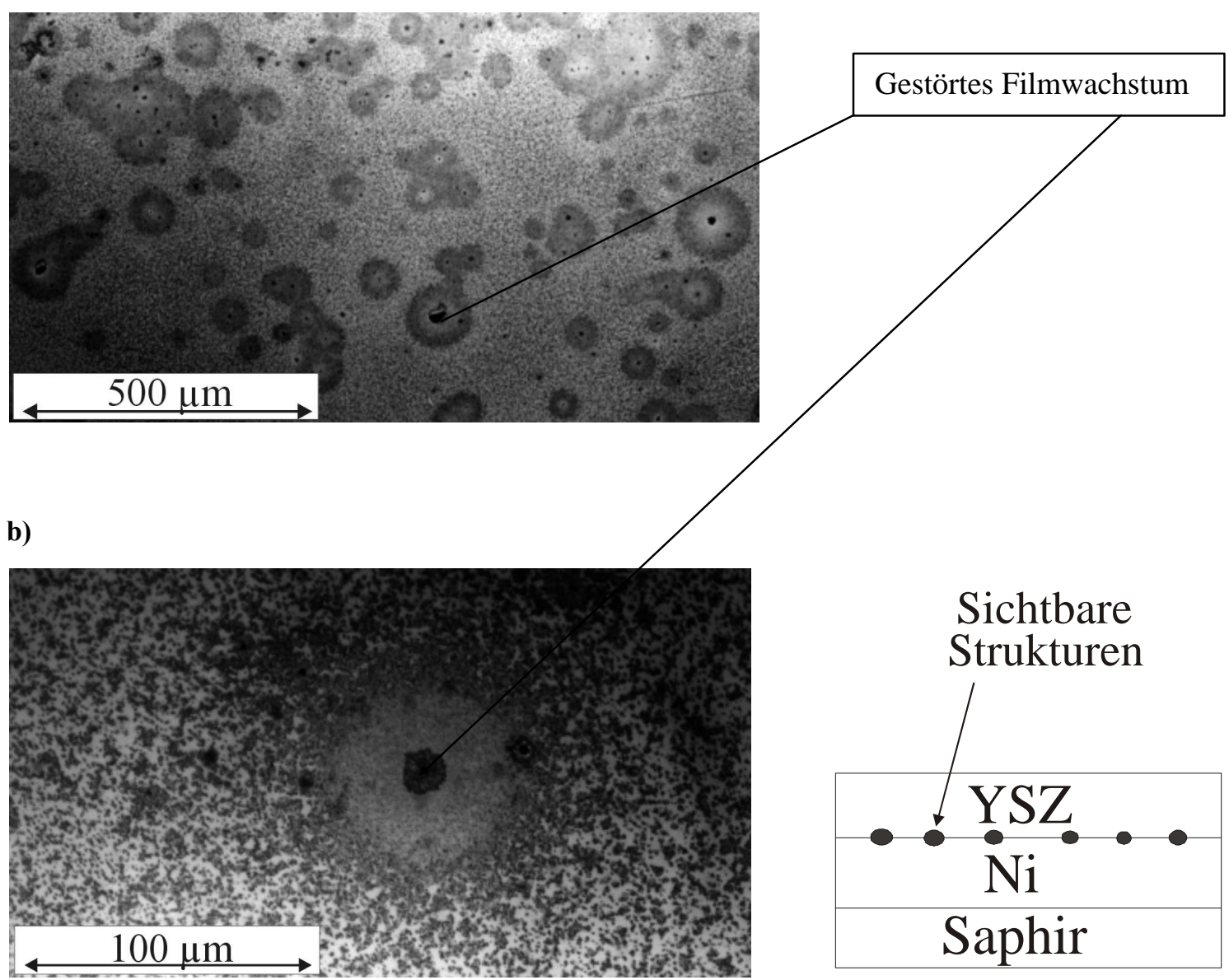

Abb 3.4a,b lichtmikroskopische Aufnahme eines Ni-YSZ-Schichtsystems nach 30 minütiger Oxidation bei $600^{\circ} \mathrm{C}$ und 1 mbar $\mathrm{O}_{2}$. Die sichtbaren Strukturen befinden sich an der Grenzfläche Ni-YSZ wie in der rechten Abbildung dargestellt. 
An der gesamten Grenzfläche befinden sich dunkle Partikel, diese sind wahrscheinlich NiO-Ausscheidungen. An Stellen, an denen das Filmwachstum des YSZ gestört ist, liegen die dunklen Partikel in hoher Dichte vor und sind kreisförmig um den Defekt angeordnet. Die in den Abbildungen 3.4a,b dargestellten Grenzflächen wurden unabhängig von der Dicke und der Textur der YSZ-Schicht beobachtet.

Weder die REM-Aufnahmen noch die lichtmikroskopischen Aufnahmen der bei 1 mbar $\mathrm{O}_{2}$ ausgelagerten Modellschichtsysteme mit den NiCr-Substraten zeigen eine Oxidbildung oder Risse.

Da die verwendeten Modellsubstrate gesputtert wurden, sind sie sehr glatt und feinkristallin. Nicht alle tatsächlich verwendeten Substrate entsprechen diesen Bedingungen. RABiTS-Bänder z.B. weisen große quasieinkristalline Körner auf, deren Korngrenzen zum Teil eingekerbt (grooving) sind. In dem folgenden Experiment wird deshalb eine Substratoberfläche hergestellt, die eine starke Oberflächenstruktur aufweist. Dazu wird ein polykristallines Ni-Band mit einem Ionenstrahl 5 Minuten lang mit einer Energie von $1000 \mathrm{~V}$ beschossen, dieses Band wird mittels Magnetronsputterns mit Nickel beschichtet, damit die Qualität der Nickelschicht mit denen der vorher betrachteten identisch ist, und auf diese Schicht wird mittels IBAD eine $300 \mathrm{~nm}$ Dicke YSZ-Schicht abgeschieden (outof-plane Textur: $9^{\circ}$; in-plane Textur: $38^{\circ}$ FWHM). Eine REM-Aufnahme dieses Schichtsystems ist in Abbildung 3.5 dargestellt.

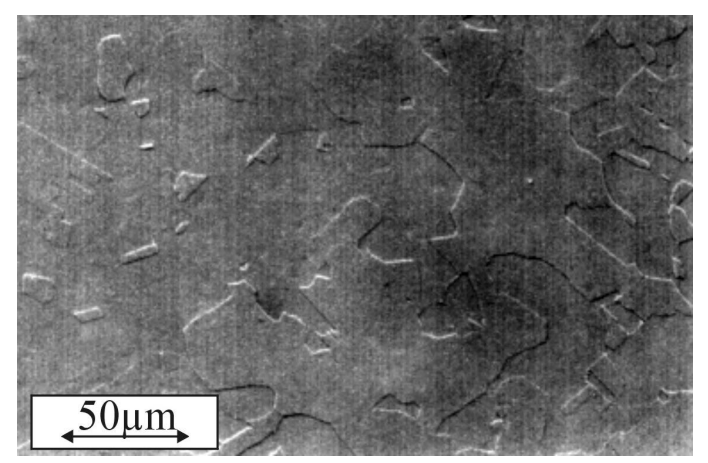

Abb. 3.5 REM-Aufnahme des mit Nickel und YSZ beschichteten polykristallinen Nickelbandes. Die stufenartige Struktur wurde mittels Ionenbeschuss erzeugt.

Die stufenartige Struktur entstand durch den Ionenbeschuss, da verschieden orientierte Körner unterschiedlich stark abgetragen werden. Diese stufenartige Struktur wird bei den Beschichtungen bis in den YSZ-Film übertragen. Dieses Schichtsystem wird im Hochvakuum bei einer Temperatur von $400^{\circ} \mathrm{C} 30$ Minuten lang ausgelagert. Die Abbildung 3.6 zeigt eine REM-Aufnahme des ausgelagerten Schichtsystems. Deutlich zu erkennen ist, dass an den Stufen und zwar nur an den Stufenstrukturen ein Abplatzen des Filmes eingesetzt hat. 


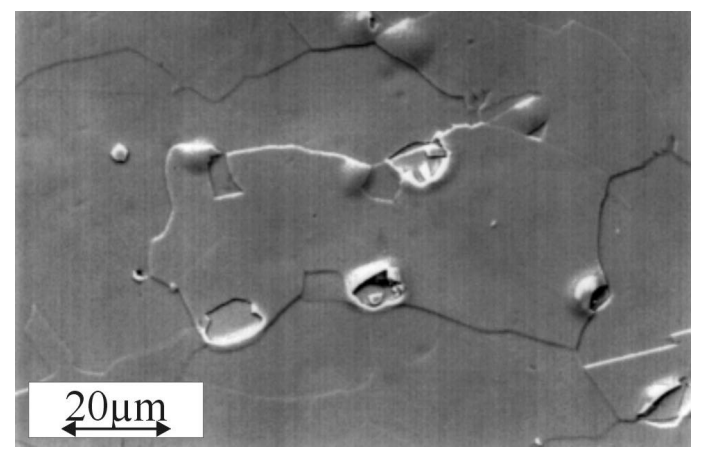

Abb. 3.6 REM-Aufnahme des mit Nickel und IBAD-YSZ beschichteten polykristallinen Nickelbandes nach einer 30 minütigen Auslagerung bei $400^{\circ} \mathrm{C}$ im Hochvakuum.

\subsection{Diskussion}

\subsubsection{Einfluss der thermischen Ausdehnung auf das Ablösen von Puffer- schichten}

Die thermischen Ausdehnungen für die verschiedenen verwendeten Materialien sind in Abbildung 3.7 dargestellt.

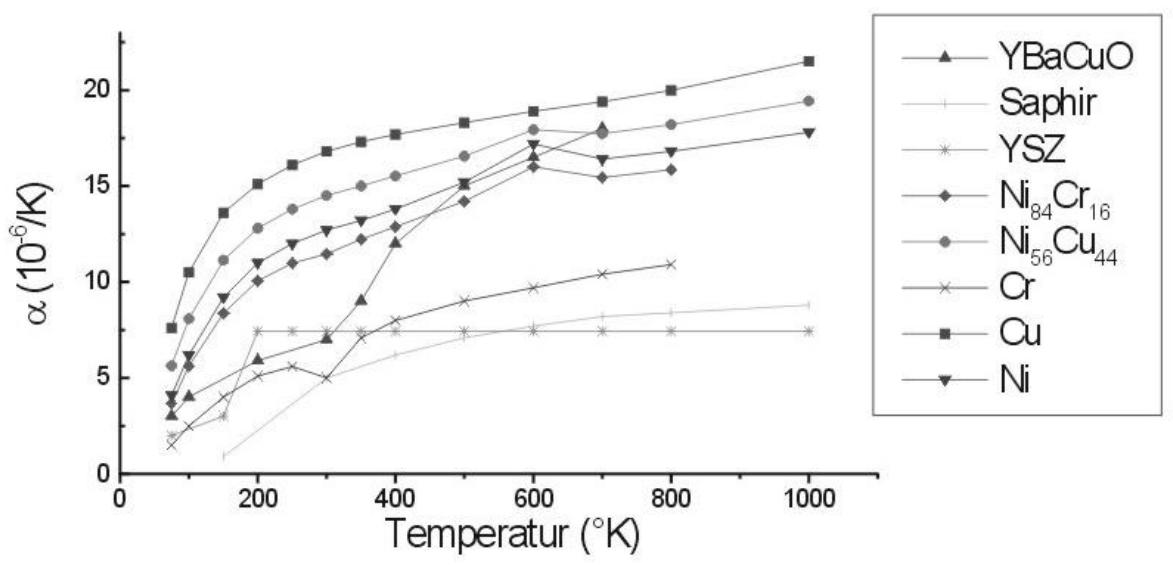

Abb 3.7 thermische Ausdehnungskoeffizienten der verwendeten Materialien [31,32]. 
Für die Ni-Legierungen wurden die thermischen Ausdehnungskoeffizienten aus denen der reinen Elementen berechnet. Die relative Dehnung zwischen Substrat und Film ist definiert als

$\varepsilon=\mathrm{dl} / \mathrm{l}=\int\left(\alpha_{\text {Film }}-\alpha_{\text {Substrat }}\right) \mathrm{dT}$.

Die relative Dehnung zwischen dem YSZ-Film und den unterschiedlichen Substratlegierungen wurden aus den oben dargestellten thermischen Ausdehnungskoeffizienten für den Temperaturbereich zwischen $300 \mathrm{~K}$ und $1000 \mathrm{~K}$ abgeschätzt und sind in Tabelle 3.1 angegeben.

\begin{tabular}{|l|l|}
\hline & Relative Dehnung $\varepsilon(\%)$ \\
\hline YSZ-Ni & $-0,53$ \\
\hline YSZ-Ni $_{56} \mathrm{Cu}_{44}$ & $-0,67$ \\
\hline YSZ-Ni $_{84} \mathrm{Cr}_{16}$ & $-0,64$ \\
\hline
\end{tabular}

Tabelle 3.1 Relative Dehnung zwischen YSZ-Pufferschicht und unterschiedlichen Substraten mit Temperaturbereich zwischen $300 \mathrm{~K}$ und $1000 \mathrm{~K}$.

Die Dehnungen zwischen dem YSZ-Puffer und den Substraten sind relativ hoch, trotzdem sind die im Hochvakuum ausgelagerten auf Saphir gesputterten Modellsubstratschichtsysteme hinsichtlich einer Temperaturbehandlung bis $700^{\circ} \mathrm{C}$ stabil, es wurde weder ein Abplatzen noch Risse oder Mikrorisse beobachtet. Die YSZ-Schicht auf dem Modellsubstratschichtsystem mit der stufenartigen Oberfläche dagegen beginnt schon bei einer Temperatur von $400^{\circ} \mathrm{C}$ an den Stufen abzuplatzen. An Stufen haben die Spannungen im Gegensatz zu den flachen Stellen auch eine Komponente senkrecht zur Filmoberfläche, die hier offenbar hoch genug ist, um ein Ablösen der Pufferschicht hervorzurufen. Aber nicht nur die verschiedenen thermischen Ausdehnungen verursachen Kräfte, die zum Abplatzen führen können, sondern ebenfalls die Relaxation des Gitters des YSZ. YSZ wird bei Raumtemperatur abgeschieden und wächst unter starken Druckspannungen auf, so dass die Gitterkonstante um ungefähr 0,13 $\AA$ aufgeweitet ist [30] . Bei einer Auslagerungstemperatur von $400^{\circ} \mathrm{C}$ relaxiert die Gitterkonstante auf ungefähr den Bulkwert des YSZ, der bei 5,13 Å liegt. Außerdem setzt bei $400^{\circ} \mathrm{C}$ verstärkt das Kornwachstum des Nickels ein. Diese Effekte zusammen verbunden mit einer Unregelmäßigkeit der Substratoberfläche können also zum Abreißen einer Pufferschicht führen.

Deshalb ist bei der Herstellung von Substratbändern zu beachten, dass eine möglichst glatte strukturlose ungestörte Oberfläche entsteht, welche, wie die anderen Modellsubstratsysteme ja gezeigt haben, diesen Kräften standhält. 
Ein weitaus größeres Problem bei der Herstellung stabiler Pufferschichten allerdings stellt die Substratoxidation dar.

\subsubsection{Einfluss der Substratoxidation auf das Ablösen von Pufferschichten}

Wie die Röntgendiffraktogramme zeigen, hat nach der Auslagerung bei $700^{\circ} \mathrm{C}$ und $1 \mathrm{mbar}$ $\mathrm{O}_{2}$ eine Oxidation der $\mathrm{Ni}$ und $\mathrm{NiCu}$-Substrate stattgefunden. Es hat also ein Sauerstofftransport durch die YSZ-Schicht stattgefunden. YSZ ist $\mathrm{ZrO}_{2}$, zu dem $8 \mathrm{Mol} \% \mathrm{Y}_{2} \mathrm{O}_{3}$ dazugegeben wurde, dies entspricht ungefähr 15 At \% Y. Durch diese Zugabe wird die kubische Phase des $\mathrm{ZrO}_{2}$ bis auf Raumtemperatur stabilisiert. Bei der Dotierung werden $\mathrm{Zr}^{4+}$ Kationen durch niedervalente $\mathrm{Y}^{3+}$ Ionen ersetzen, der dabei entstehende negative Ladungsüberschuß wird durch die Bildung von strukturellen Sauerstoffleerstellen kompensiert. Bei einer Zugabe von $8 \mathrm{Mol} \% \mathrm{Y}_{2} \mathrm{O}_{3}$ fehlt jedes 27. Sauerstoffatom. Deshalb ist YSZ ein guter Sauerstoffionenleiter [33].

\subsubsection{Oxidation des Nickels}

Die Röntgendiffraktogramme der Modellschichtsysteme mit dem Nickelsubstraten zeigen deutliche Nickeloxidreflexe. Die Intensität der Nickeloxidreflexe ist bei beiden Schichtsystemen, dem Schichtsystem mit der gut texturierten dicken YSZ-Schicht und der weniger gut texturierten dünnen YSZ-Schicht, gleich groß. Die Erklärung für die gleichhohen Intensitäten liefern die lichtmikroskopischen Aufnahmen der Schichtsysteme. Auf ihnen ist zu erkennen, dass die Filme hauptsächlich dort oxidieren, an denen das Filmwachstum gestört ist, und dass die Oxidbildung unterhalb des unversehrten Puffers vergleichsweise gering ist. Für die Substratoxidation ist also nicht Dicke oder die Qualität der Textur des Puffers entscheidend, sondern die Anzahl der gestörten Defekte im Puffer. Diese sind offenbar für Sauerstoff so durchlässig, dass die Oxidationskinetik den gebildeten NiO-Anteil bestimmt und nicht die Diffusion des Sauerstoffs. Da die verschiedenen YSZPufferschichten in derselben Anlage auf identisch hergestellte Modellsubstrate abgeschieden wurden, kann davon ausgegangen werden, dass die Anzahl der Störstellen auf den beiden Schichtsystemen ungefähr gleich groß ist. Das heisst, der Sauerstofftransport findet hauptsächlich nicht durch den Puffer sondern durch dessen Defekte statt. Da die REMAufnahmen keine Risse und kein Abplatzen zeigen, war die Oxidbildung nicht so stark, dass sie ein Abreißen des Filmes bewirken konnte. Aber nicht nur die Bereiche des gestörten Filmwachstums der Pufferschicht sind Schwachstellen an denen eine verstärkte Substratoxidation stattfindet. So beobachtete F.G. Moreno [34] bei der Auslagerung von 
mit dünnen YSZ-Filmen beschichtenen Ni-Bändern unter YBaCuO-Depositionsbedingungen eine Oxidation des Ni-Substrates lediglich an dessen Korngrenzen.

\subsubsection{Oxidation des $\mathrm{Ni}_{85} \mathrm{Cr}_{15}$}

Die Oxidation von einer $\mathrm{Ni}_{85} \mathrm{Cr}_{15}$-Legierung kann folgendermaßen beschrieben werden [35]. Nickel ist die edlere Legierungskomponente, $\mathrm{NiO}$ aber bildet sich sehr viel schneller als $\mathrm{Cr}_{2} \mathrm{O}_{3}$. Deshalb bildet sich zu Beginn der Oxidation eine NiO-Schicht auf der Legierung. An der Grenzschicht $\mathrm{NiO}-\mathrm{Ni}_{85} \mathrm{Cr}_{15}$ kommt es zur Anreicherung von $\mathrm{Cr}_{2} \mathrm{O}_{3}$, da dort die Sauerstoffaktivität so herabgesetzt ist, dass der Dissoziationsgleichgewichtsdruck zur Bildung von $\mathrm{NiO}$ unterschritten ist, so findet hier eine selektive Oxidation des Chroms statt und es bildet sich eine Chromoxidschicht. Die Diffusion von Nickel durch die Chromoxidschicht ist vernachlässigbar klein, deshalb wird die äußere NiO-Schicht von einer Chromoxidschicht unterwachsen. Die Chromoxidschicht wächst nach außen und aufgrund ihrer hohen Dichte und geringen Fehlstellenkonzentration sehr langsam und stellt einen guten Korrosionsschutz dar (siehe hierzu Kapitel 6.1.2).

Thomas Simon [36] führte Oxidationsexperimente an Hastelloy-YSZ-Schichten durch. Hastelloy C22 setzt sich aus den folgenden Elementen zusammen: Ni 53-55 At \%, Cr 2022,5 At \%, Mo 12,5-14,5 At \%, Fe 2-6 At \%, weitere Elemente $<3$ At \%. Das Oxidationsverhalten von Hastelloy lässt sich in etwa mit dem von einer $\mathrm{Ni}_{85} \mathrm{Cr}_{15}$ Legierung vergleichen. Ein Hastelloysubstrat wurde mit einer untexturierten $250 \mathrm{~nm}$ dicken YSZ-Schicht und ein weiteres mit einer texturierten $50 \mathrm{~nm}$ dicken IBAD-YSZ-Schicht beschichtet. Die Schichtsysteme wurden 45 Minuten bei $740^{\circ} \mathrm{C}$ und 0,6 mbar $\mathrm{O}_{2}$ oxidiert und anschließend mittels Augerelektronentiefenprofilanalysen untersucht. Unter der untexturierten YSZSchicht hatte sich eine $100 \mathrm{~nm}$ dicke NiO-Schicht gebildet und darunter eine $160 \mathrm{~nm}$ dicke $\mathrm{Cr}_{2} \mathrm{O}_{3}$-Schicht, während sich unter der mit IBAD texturierten Schicht lediglich eine $30 \mathrm{~nm}$ dicke $\mathrm{Cr}_{2} \mathrm{O}_{3}$-Schicht gebildet hatte. Das unterschiedliche Oxidationsverhalten wird anhand des unterschiedlichen Sauerstofftransports durch die verschiedenen YSZ-Schichten erklärt. Die YSZ-Schichten unterscheiden sich im wesentlichen durch die Korngrenzenstrukturen. Die mittels IBAD texturierte YSZ-Schicht ist wesentlich dichter und hat keine Großwinkelkorngrenzen. Der Sauerstofftransport durch die texturierte YSZ-Schicht ist also so gering, dass der Dissoziationsgleichgewichtdruck zur Bildung von $\mathrm{NiO}$ unterschritten ist und somit nur das unedlere Chrom oxidieren kann. Durch die untexturierte YSZ-Schicht findet ein sehr viel größerer Sauerstofftransport statt, der Sauerstoffaktivität liegt an der Grenzfläche zum Substrat immer noch über dem Dissoziationsgleichgewichtsdruck vom NiO, das Nickel oxidiert und diese Schicht wird von einer Chromoxidschicht unterwachsen. Der Sauerstofftransport geschieht also im wesentlichen über die Korngrenzen des YSZ. 
Die in dieser Arbeit oxidierten $\mathrm{Ni}_{84} \mathrm{Cr}_{16}$-YSZ-Schichten zeigen keine Oxidreflexe und auch keine Oxidausscheidungen auf den lichtmikroskopischen und REM-Aufnahmen. Da die Schichtsysteme mit den Ni-Filmen als Substrat aber deutlich zeigen, dass eine Oxidation des Nickels stattgefunden hat und da der Sauerstofftransport durch die identisch hergestellten Pufferschichten derselbe sein sollte, ist davon auszugehen, dass sich eine dünne Chromoxidschicht gebildet hat, diese kann jedoch mit den hier benutzten experimentellen Methoden nicht nachgewiesen werden.

\subsubsection{Oxidation des $\mathrm{Ni}_{56} \mathrm{Cu}_{44}$}

Die Oxidationsgeschwindigkeit in Abhängigkeit von der Legierungszusammensetzung des $\mathrm{NiCu}$ wurde von Pilling und Bedworth [37,35] untersucht. Sie oxidierten NiCu Legierungen mit unterschiedlichen Zusammensetzungen bei $950^{\circ} \mathrm{C}$ eine Stunde lang an Luft und bestimmten die Oxidschichtdicke (Abb. 3.8).

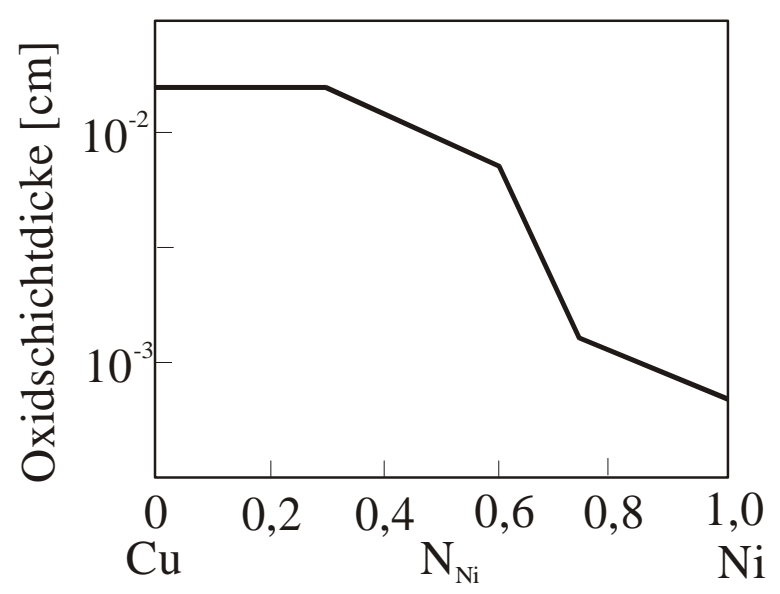

Abb. 3.8 Oxidschichtdicke gegen den Anteil an Nickel nach einer einstündigen Oxidation an Luft bei $950^{\circ} \mathrm{C}[37]$.

Wie die Abbildung 3.8 zeigt, beschleunigt die Zulegierung von Kupfer zu Nickel die Oxidation erheblich. Die Oxidschichtdicke einer $\mathrm{Ni}_{50} \mathrm{Cu}_{50}$-Legierung ist ungefähr eine Größenordnung höher als die von reinem Nickel, obwohl Kupfer das edlere Element ist. Carl Wagner [38] erklärt diesen Prozess am Beispiel von NiCu-Legierungen mit einem Kupferanteil geringer als 25 At \%. Zu Beginn der Oxidation bildet sich an der Oberfläche außer dem $\mathrm{NiO}$ stellenweise $\mathrm{Cu}_{2} \mathrm{O}$. Da die Beweglichkeit von $\mathrm{Cu}^{+}$-Ionen in $\mathrm{Cu}_{2} \mathrm{O}$ sehr viel höher ist als die entsprechende Beweglichkeit von $\mathrm{Ni}^{2+}$ in $\mathrm{Cu}_{2} \mathrm{O}$ und in $\mathrm{NiO}$, wächst das $\mathrm{Cu}_{2} \mathrm{O}$ schneller weiter als das entstandene $\mathrm{NiO}$ (Abb. 3.9a). 
a)

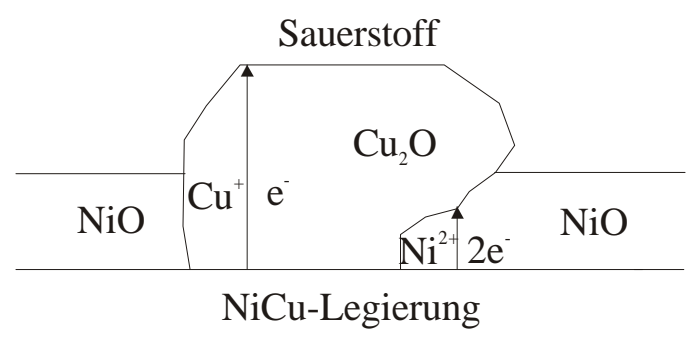

b)

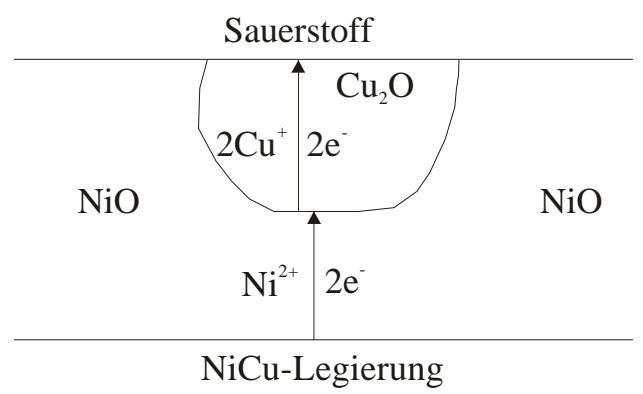

Abb. 3.9 a,b Schematische Zeichnungen der Oxidation einer NiCu-Legierung nach C. Wagner [38].

Da NiO thermodynamisch die stabilere Phase ist, setzt sich das $\mathrm{Cu}_{2} \mathrm{O}$ an der Grenzfläche zur Legierung im weiteren Verlauf der Oxidation in $\mathrm{NiO}$ und $\mathrm{Cu}^{+}$-Ionen um (Abb. 3.9b).

$\mathrm{Ni}^{2+}+2 \mathrm{e}^{-}+\mathrm{Cu}_{2} \mathrm{O} \rightarrow \mathrm{NiO}+2 \mathrm{Cu}^{+}+2 \mathrm{e}^{-}$

Die freiwerdenden Kupferionen diffundieren zur äußeren Grenzfläche und bilden dort mit chemisorbiertem Sauerstoff neues $\mathrm{Cu}_{2} \mathrm{O}$, so wachsen die $\mathrm{Cu}_{2} \mathrm{O}$-Inseln nach außen. Aufgrund der Umsetzungsreaktion geht der Kontakt zwischen $\mathrm{Cu}_{2} \mathrm{O}$ und der $\mathrm{NiCu}-$ Legierung im Verlauf der Reaktion verloren. Durch die sehr viel raschere Bildung des $\mathrm{Cu}_{2} \mathrm{O}$ ist die

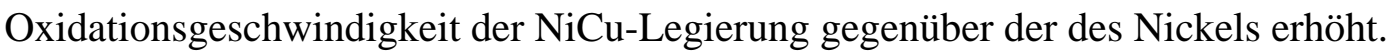

Bei höheren Mengen an Kupfer ( höher als 25 At \% Cu) werden Kupferoxiddeckschichten gefunden $[39,40]$. Je nachdem in welchem Druckbereich oxidiert wird, bilden sich $\mathrm{CuO}$ oder $\mathrm{Cu}_{2} \mathrm{O}$ Deckschichten. Aber auch, wenn im Druckbereich der Bildung von $\mathrm{Cu}_{2} \mathrm{O}$ oxidiert wird, sind über den $\mathrm{Cu}_{2} \mathrm{O}$-Deckschichten häufig dünne $\mathrm{CuO}$-Schichten zu beobachten.

Die Röntgendiffraktogramme des bei $700^{\circ} \mathrm{C}$ und $1 \mathrm{mbar} \mathrm{O}_{2}$ ausgelagerten Modellschichtsystems mit dem $\mathrm{Ni}_{56} \mathrm{Cu}_{44}$-Substrat zeigen deutlich $\mathrm{CuO}$-Reflexe und $\mathrm{NiO}$-Reflexe, die im Vergleich zu den oxidierten Ni-Modellschichtsubstraten sehr hohe Intensitäten aufweisen. Die REM-Aufnahmen zeigen, dass schon bei einer Temperatur von $500^{\circ} \mathrm{C}$ der YSZ-Film an vielen Stellen abgeplatzt ist. Vermutlich hat sich dies an Stellen ereignet, an denen das Filmwachstum des YSZ gestört war, da dort, wie die Experimente mit den Nickelmodellsubstratschichtsystemen zeigen, der Sauerstofftransport zum Substrat am höchsten ist. Wie die Elementverteilungsbilder zeigen, ist an den abgeplatzten Stellen eine erhöhte Konzentration an Kupfer und Sauerstoff zu beobachten. Dies spricht dafür, dass sich direkt an der Grenzfläche zum YSZ $\mathrm{CuO}$ befindet und das $\mathrm{NiO}$ erst unterhalb dieser $\mathrm{CuO}$-Schicht auftritt. Die Kupferoxidbildung hat dann das Abreißen des YSZ-Filmes zur Folge. Wie bereits erwähnt, ist es bei der Oxidation des Legierungsystem $\mathrm{NiCu}$ charakteristisch, dass sich $\mathrm{CuO}$ an der Oberfläche befindet und sehr viel schneller wächst als das $\mathrm{NiO}$, deshalb ist ein 
durch die Oxidbildung bedingtes Abplatzen des YSZ-Films auf einem NiCu-Substrat auch schon bei niedrigeren Temperaturen zu erwarten als auf einem Nickelsubstrat. Bei einer weiteren Auslagerung bei $600^{\circ} \mathrm{C}$ sind die Stellen, die schon abgeplatzt waren und an denen sich schon CuO-Inseln befunden haben, gewachsen. Die mittlere Größe der CuO-Inseln erhöhte sich von $13 \mu \mathrm{m}$ auf $27 \mu \mathrm{m}$. Da an diesen Stellen die YSZ-Schicht schon abgeplatzt war, stehen die CuO-Inseln bei der weiteren Oxidation in direkter Verbindung zum Sauerstoff und wachsen lateral weiter. Zwischen den großen abgeplatzten Bereichen sind nun auch kleine Bereiche zu beobachten, die abreißen (Abb 3.2b). Offensichtlich ist bei $600^{\circ} \mathrm{C}$ nun auch der Sauerstofftransport durch den unversehrten YSZ-Puffer groß genug für eine Kupferoxidbildung, die zum Abplatzen des Films führt. Eine weitere Auslagerung der Schicht bei $700^{\circ} \mathrm{C}$ zeigt eine weitere Vergrößerung der abgeplatzen Bereiche auf $32 \mu \mathrm{m}$. Die Anzahl der kleineren abgeplatzten Bereiche hat weiter zugenommen.

\subsection{Schlussfolgerung}

Das wesentliche Problem bei der YBaCuO-Deposition auf gepufferten metallischen Substratbändern stellt die Oxidation des Substrats dar, die im schlimmsten Fall zum Abplatzen des Filmes führen kann. Da die meisten verwendeten Puffermaterialien keramische Oxide sind, findet bei diesen auch immer ein Sauerstofftransport zum Substrat hin statt. T. Simon hat gezeigt, dass sich dieser erheblich durch eine gute Textur der Pufferschicht reduzieren lässt. Wie die oben dargestellten Ergebnisse zeigen, findet aber auch ein Sauerstofftransport durch Bereiche gestörten Filmwachstums statt, der von der Dicke und der Textur der Pufferschicht unabhängig ist. Da solche Defekte aber nicht vollständig vermieden werden können, ist es notwendig, ein weitgehend oxidationsbeständiges Substratmaterial zu verwenden. Soll auf Ni-Legierungen zurückgeriffen werden, so zeigt $\mathrm{Ni}_{84} \mathrm{Cr}_{16}$ ein Oxidationsverhalten, das unter der Nachweisgrenze der hier verwendeten experimentellen Methoden liegt, und ist somit ein sehr geeignetes Substratmaterial. $\mathrm{Ni}_{56} \mathrm{Cu}_{44}$ dagegen hat eine im Vergleich zum Nickel erhöhte Oxidationsgeschwindigkeit und ist damit noch ungeeigneter als das Nickel selbst. Bei der Verwendung von RABiTS-Bändern als Substrat führen zusätzlich die Korngrenzen, die teilweise eingekerbt sind (grooving) zu Schwachstellen, da hier schon die thermischen Spannungen zum Abriss der schützenden Pufferschicht führen können.

Da eine Oxidation des Substrates während der YBaCuO-Deposition nicht vollständig vermieden werden kann, bietet sich eine gezielte epitaktische Oxidation an, so dass NiO entweder selber als Pufferschicht bzw. als Unterlage für die Abscheidung weiterer Pufferschichten wirkt. In den folgenden Kapiteln wird deshalb diese gezielte epitaktische Oxidation näher untersucht. 


\section{Oxidation von RABiTS-Bändern unter hohen Temperaturen}

Bisher wurden Pufferschichten für den $\mathrm{YBaCuO}$ Supraleiter entweder durch das IBADVerfahren, d.h. durch Ionenbeschuss während der Pufferabscheidung biaxial texturiert, oder es wurden meist keramische Oxide epitaktisch auf vortexturierten Substraten (RABiTS) abgeschieden. In diesem Kapitel wird ein alternatives Verfahren zur Pufferschichtherstellung vorgestellt, nämlich die epitaktische Oxidation. Dabei soll auf vortexturierten $\{100\}<100>\mathrm{Ni}$ und Ni-Legierungen durch Oxidation NiO epitaktisch und möglichst homogen aufwachsen und als Pufferschicht fungieren. Dieses Verfahren wäre sehr viel schneller, kostengünstiger und mit weniger apparativem Aufwand durchzuführen als die bisher verwendeten. Aus diesem Grund wurden auch die folgenden Experimente unter den denkbar einfachsten Bedingungen durchgeführt.

$\{100\}<100>$ texturierte $\mathrm{Ni}^{-}, \mathrm{Ni}_{87} \mathrm{Cr}_{13^{-}}$, und $\mathrm{Ni}_{91} \mathrm{~V}_{9^{-}}$RABiTS-Bänder wurden in einem gasgespültem Rohrofen bei verschiedenen Temperaturen $\left(1050^{\circ} \mathrm{C}, 1150^{\circ} \mathrm{C}\right.$ und $\left.1250^{\circ} \mathrm{C}\right)$ oxidiert. Diese experimentelle Anordnung ist für die Oxidation von Bändern großer Länge geeignet. Die wichtigsten Parameter der RABiTS-Bänder sind in Tabelle 4.1 dargestellt. Die verwendeten Bänder wiesen ähnliche Texturen aber unterschiedliche Rauigkeiten und Korngrößen auf.

\begin{tabular}{|c|c|c|c|c|}
\hline & $\begin{array}{c}\text { Out-of-plane } \\
\text { Textur (FWHM) }\end{array}$ & $\begin{array}{c}\text { In-plane } \\
\text { Textur (FWHM) }\end{array}$ & $\begin{array}{c}\text { Rauigkeit } \\
(\mathrm{nm})\end{array}$ & $\begin{array}{c}\text { Mittlere } \\
\text { Korngröße }(\mu \mathrm{m})\end{array}$ \\
\hline $\mathrm{Ni}$ & $7^{\circ}$ & $9^{\circ}$ & 460 & 100 \\
\hline $\mathrm{Ni}_{87} \mathrm{Cr}_{13}$ & $7^{\circ}$ & $8^{\circ}$ & 70 & 50 \\
\hline $\mathrm{Ni}_{91} \mathrm{~V}_{9}$ & $7^{\circ}$ & $8^{\circ}$ & 80 & 40 \\
\hline
\end{tabular}

Tab. 4.1 Texturen, Rauigkeiten und mittlere Korngrößen der RABiTS-Bänder.

Der Rohrofen wurde dabei, auch während der Aufheiz-und Abkühlphase, entweder mit reinem Argon oder reinem Sauerstoff oder mit einem Gasgemisch aus Argon und Sauerstoff durchflossen, wobei das Mischungsverhältnis ungefähr $10 \mathrm{zu} 1$ war. Genaue Sauerstoffpartialdrücke konnten aufgrund der experimentellen Anordnung nicht angegeben werden, aber die Bedingungen konnten reproduziert werden. Die Heizrate betrug $900^{\circ} \mathrm{C} / \mathrm{h}$, die 
Abkühlphase dauerte ungefähr 3-4 Stunden. Der Zeitraum, über den die Endtemperatur gehalten wurde, betrug $5 \mathrm{~min}$.

\subsection{Die Phasenbildung}

Nach der Oxidation wurden $\Theta-2 \Theta-$ Röntgendiffraktogramme von allen Proben gemessen.(Abb.4.1-4.3) Im untersuchten Parameterbereich wird fast ausschließlich die NiOPhase gebildet. Die charakteristischen Reflexe dieser Struktur zeigt die Abbildung 4.1. Für die Bänder mit Legierungszusatz wird zudem die Bildung von Mischoxiden bzw. Oxiden des Zusatzes beobachtet, wobei der Volumenanteil dieser Phasen aber sehr klein ist, die Intensität der Röntgenreflexe ist typischerweise um einen Faktor 100 bis 200 kleiner als die der NiO-Phase.

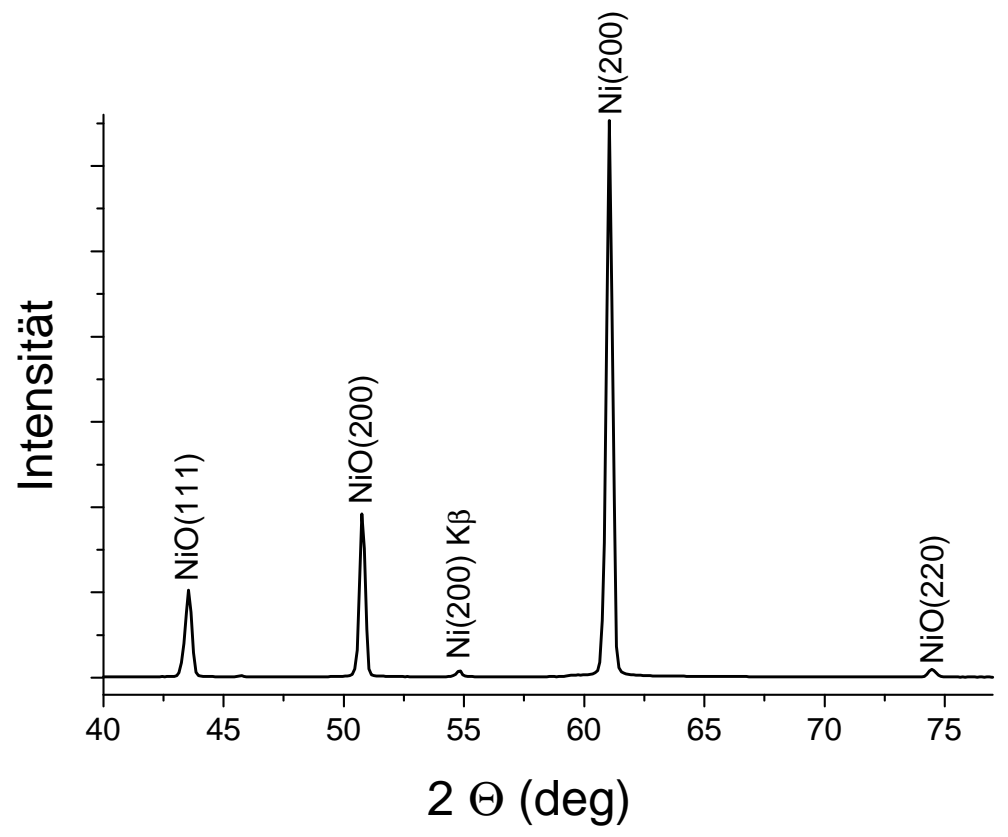

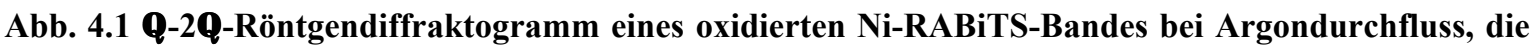
Endtemperatur betrug $1250^{\circ} \mathrm{C}$.

Die Abbildung 4.2 zeigt dies am Beispiel des NiCr. Bemerkenswert ist, dass der Anteil des Mischoxids $\mathrm{NiCr}_{2} \mathrm{O}_{4}$ nur wenig mit dem Druck variiert, während der Anteil des $\mathrm{Cr}_{2} \mathrm{O}_{3}$ mit abnehmenden Druck zunimmt. Die Oxidreflexe für die NiV-Bänder lassen sich nicht eindeutig zuordnen, aber auch hier lassen sich 2 Gruppen identifizieren, die ähnlich wie bei der Oxidation des NiCr vom Sauerstoffdruck unabhängig bzw. stark abhängig sind. 


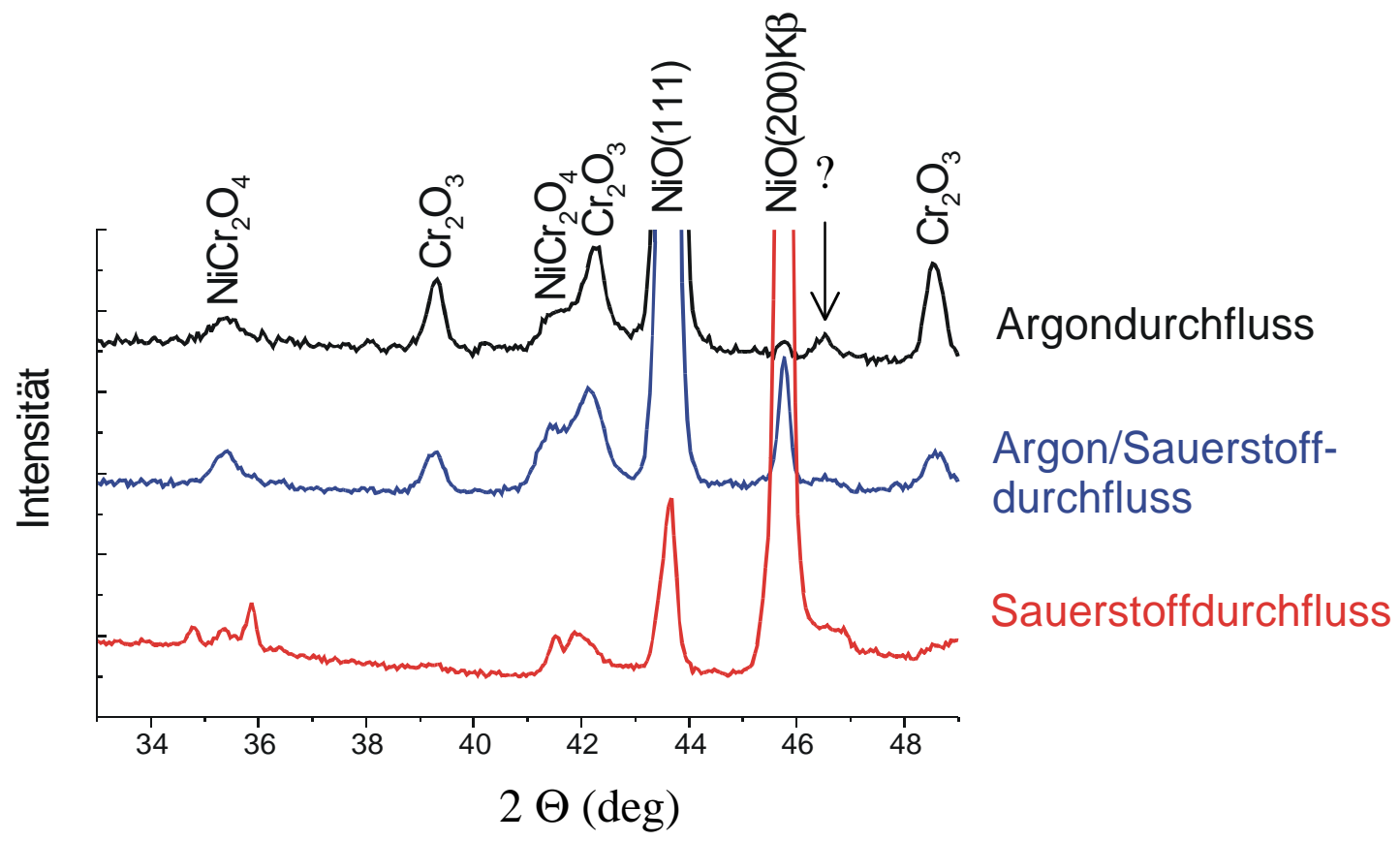

Abb. 4.2 $\Theta-2 \Theta$ Röntgendiffraktogramme der oxidierten NiCr-RABiTS-Bänder bei unterschiedlichen Sauerstoffpartialdrücken, die Endtemperatur betrug $1250^{\circ} \mathrm{C}$.

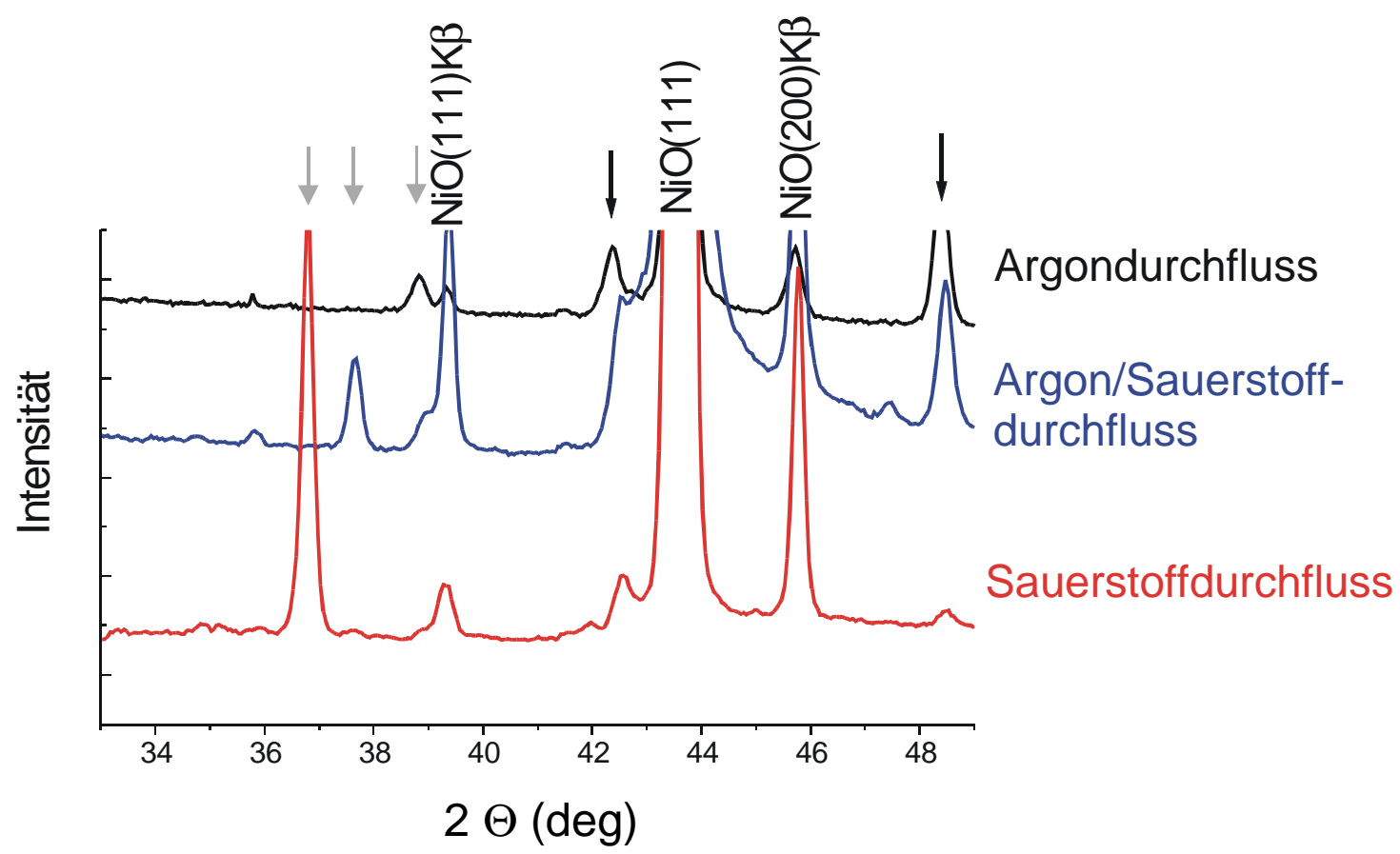

Abb. 4.3 $\Theta-2 \Theta$ Röntgendiffraktogramme der oxidierten NiV-RABiTS-Bänder bei unterschiedlichen Sauerstoffpartialdrücken, die Endtemperatur betrug $1250^{\circ} \mathrm{C}$. 


\subsection{Das epitaktische Wachstum}

Neben der gewünschten (100)-Orientierung tritt im NiO auch ein nicht zu vernachlässigender (111)-orientierter Anteil auf. Beide Orientierungen sind in-plane texturiert, es besteht also eine Epitaxiebeziehung zwischen $\mathrm{NiO}(100)$ und $\mathrm{Ni}(100)$ bzw. $\mathrm{NiO}(111)$ und Ni(100). Der Begriff Epitaxie soll hier und im folgenden als das Auftreten einer ausgeprägten Vorzugsorientierung des aufgewachsenden Materials relativ zum Ausgangsmaterial verstanden werden.

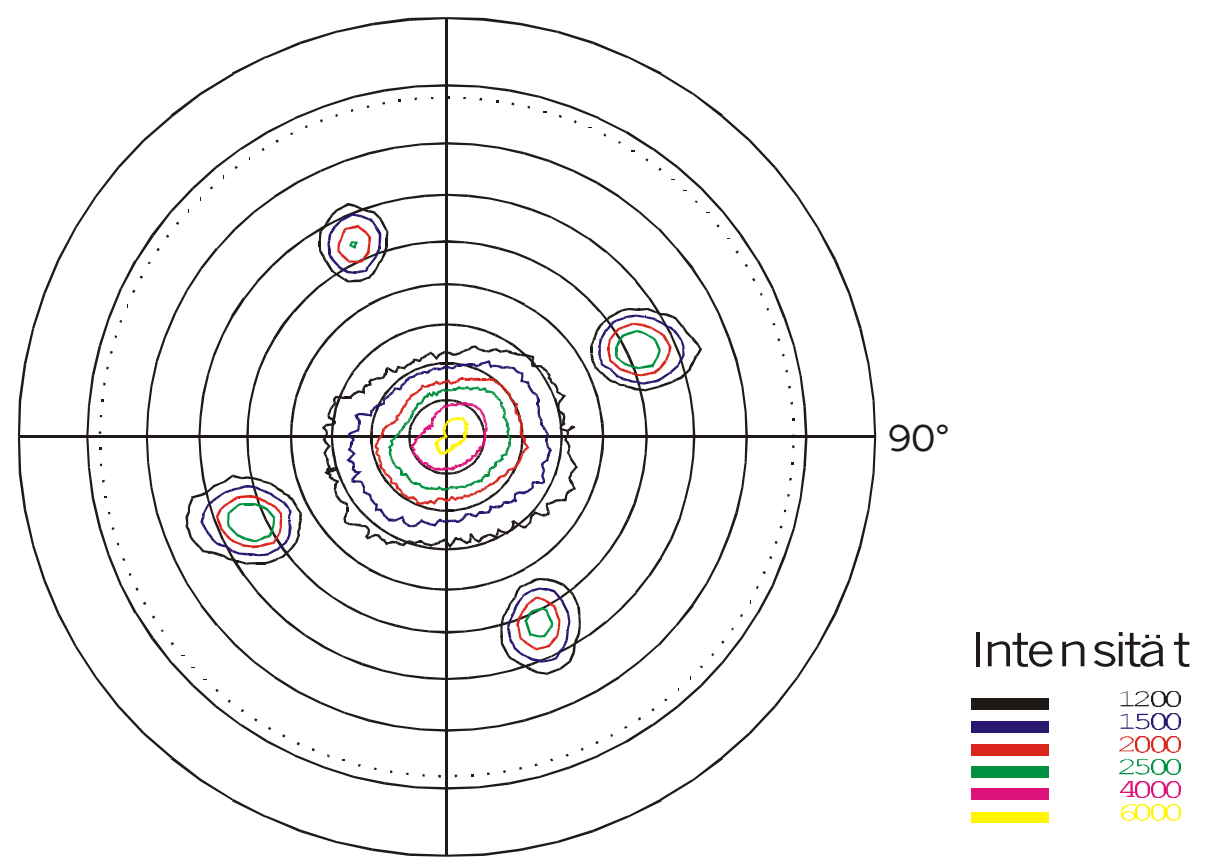

Abb. 4.4 Polfigur des NiO (111)-Reflexes eines unter Argondurchfluss oxidierten Ni-RABiTS-Bandes. Die Endtemperatur betrug $1250^{\circ} \mathrm{C}$.

Die Abbildung 4.4 zeigt eine Polfigur des NiO(111)-Reflexes. Für das kubisch flächenzentrierte NiO gibt diese Figur die Vorzugsrichtung des (100)-orientierten Anteils wieder. Erstaunlicherweise sind die gemessenen in-plane Texturen, die zwischen $11^{\circ}-14^{\circ} \mathrm{FWHM}$ liegen und damit ungefähr $3^{\circ}-6^{\circ}$ FWHM schlechter sind als die des RABiTS-Substrates, häufig besser als die zugehörigen out-of-plane Texturen, deren Halbwertsbreiten zwischen $10^{\circ}$ und $20^{\circ} \mathrm{FWHM}$ liegen. Aus dem Vergleich der (111)-Polfiguren des $\mathrm{NiO}$ mit den (111)-Polfiguren der zugehörigen oxidierten RABiTS-Bänder wurde beobachtet, dass sich das $\mathrm{NiO}(100)$ in-plane in zwei verschiedenen Epitaxierelationen anordnet, nämlich der $0^{\circ}$ und der $45^{\circ}$ Epitaxierelation. Zur Verdeutlichung wurden die beiden Epitaxierelationen in der Abbildung 4.5 schematisch dargestellt. 


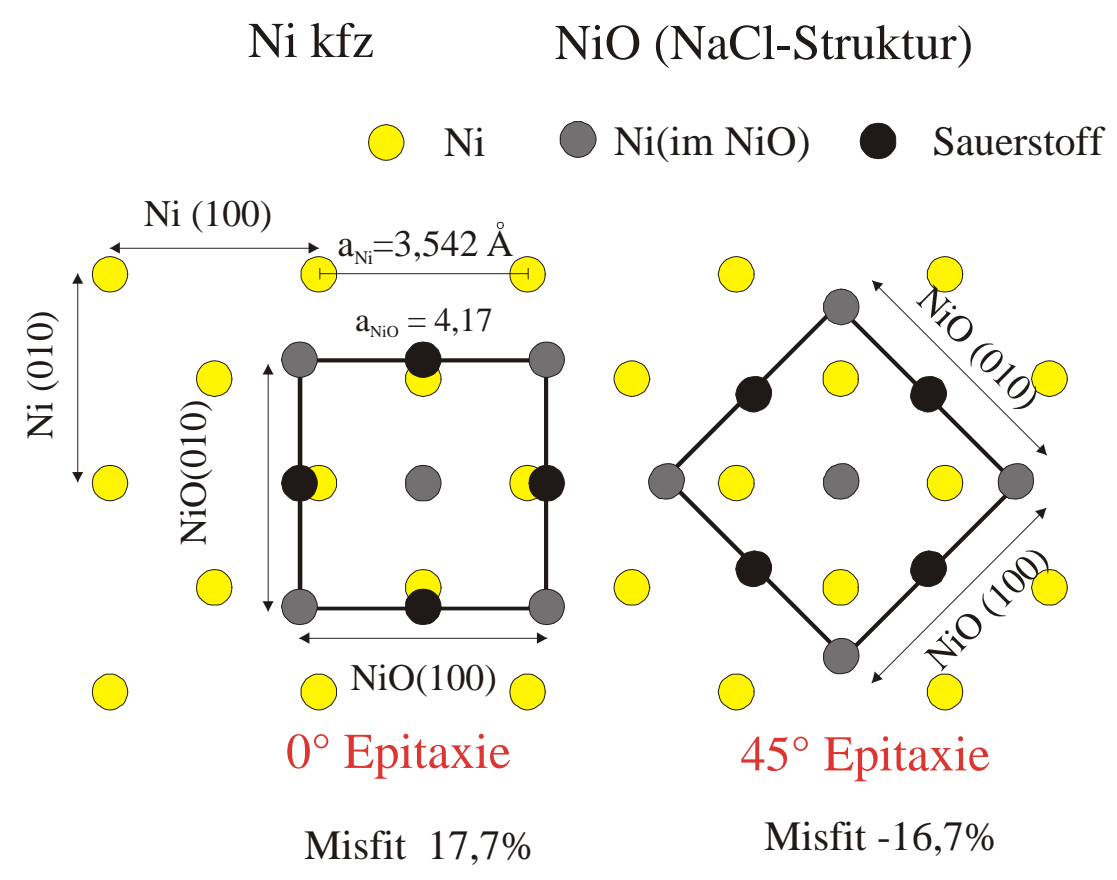

Abb. 4.5 In-plane Epitaxierelationen zwischen $\mathrm{Ni}(100)$ und $\mathrm{NiO}(100)$

Die Gitterfehlpassungen beider Anteile sind relativ hoch und vom Betrag her gleich groß, der entscheidende Unterschied ist, dass in einem Fall Druck- und im anderen Zugspannungen auftreten. Welche der beiden Epitaxierelationen sich einstellt, ist abhängig vom Sauerstoffpartialdruck während der Oxidation und von der Substratlegierung. In der Tabelle 4.2 sind die sich bildenden Epitaxierelation in Abhängigkeitvon den eben erwähnten Parametern dargestellt.

\begin{tabular}{|l|l|l|l|}
\hline & Ar-Durchfluss & Ar/O $/$ Durchfluss & $\mathrm{O}_{2}$-Durchfluss \\
\hline $\mathrm{Ni}$ & $0^{\circ}$-Epitaxie & $45^{\circ}$-Epitaxie & $45^{\circ}$-Epitaxie \\
\hline $\mathrm{Ni}_{87} \mathrm{Cr}_{13}$ & $\begin{array}{l}0^{\circ} \text {-Epitaxie } \\
\text { geringer Anteil } 45^{\circ}\end{array}$ & $\begin{array}{l}45^{\circ} \text {-Epitaxie } \\
\text { geringer Anteil } 0^{\circ}\end{array}$ & $\begin{array}{l}45^{\circ} \text {-Epitaxie } \\
\text { geringer Anteil } 0^{\circ}\end{array}$ \\
\hline $\mathrm{Ni}_{91} \mathrm{~V}_{9}$ & $0^{\circ}$-Epitaxie & $\begin{array}{l}45^{\circ} \text {-Epitaxie } \\
\text { geringer Anteil } 0^{\circ}\end{array}$ & $0^{\circ}$-Epitaxie \\
\hline
\end{tabular}

Tab. 4.2 Epitaxierelationen in Abhängigkeit vom Sauerstoffpartialdruck.

Bei Ni und der NiCr-Legierung tritt bei niedrigen Sauerstoffpartialdrücken die $0^{\circ}$ Epitaxierelation und bei höheren Sauerstoffpartialdrücken die $45^{\circ}$ Epitaxierelation auf. Ein ähnliches Verhalten findet K. Thiele [41] bei der Abscheidung von CGO (CeGdO) auf einkristallinem $\mathrm{Ni}(100)$. Hierbei tritt hauptsächlich die $0^{\circ}$-Epitaxierelation auf, wenn bei Beginn der Abscheidung kein Sauerstoffpartialdruck in die Depositionskammer eingelassen wurde, während sich hauptsächlich die $45^{\circ}$-Epitaxierelation ausbildet, wenn von Beginn der 
Deposition an ein Sauerstoffpartialdruck von $1,3 \cdot 10^{-5}$ oder $1,3 \cdot 10^{-4}$ mbar $\mathrm{O}_{2}$ eingelassen wurde. K. Thiele gibt an, dass die $45^{\circ}$-Orientierung im Vergleich zur $0^{\circ}$-Orientierung die größere Fehlpassung aber die höhere Dichte an Koinzidenzgitterplätzen aufweist. Bei geringen Sauerstoffpartialdrücken entsteht ein sauerstoffdefizitäres CGO, in dem weniger Nichtkoinzidenzplätze besetzt werden, was die $0^{\circ}$-Orientierung favorisiert. Die von $\mathrm{K}$. Thiele gegebene Erklärung lässt sich nicht auf die verschiedenen Epitaxierelationen des $\mathrm{NiO}$ übertragen. Denn zum einen sind die Gitterfehlpassungen vom Betrag her sehr ähnlich, sie beträgt für die $0^{\circ}$-Epitaxierelation $-17,7 \%$ und für die $45^{\circ}$-Epitaxierelation $16,7 \%$. Zum anderen tritt die $0^{\circ}$-Epitaxierelation bis $\mathrm{zu}$ so hohen Sauerstoffpartialdrücken auf, dass nicht mehr von Sauerstoffleerstellen ausgegangen werden kann, die NichtKoinzidenzplätze besetzen.

Die Abbildung 4.6 zeigt nun eine Polfigur des NiO(200)-Reflexes.

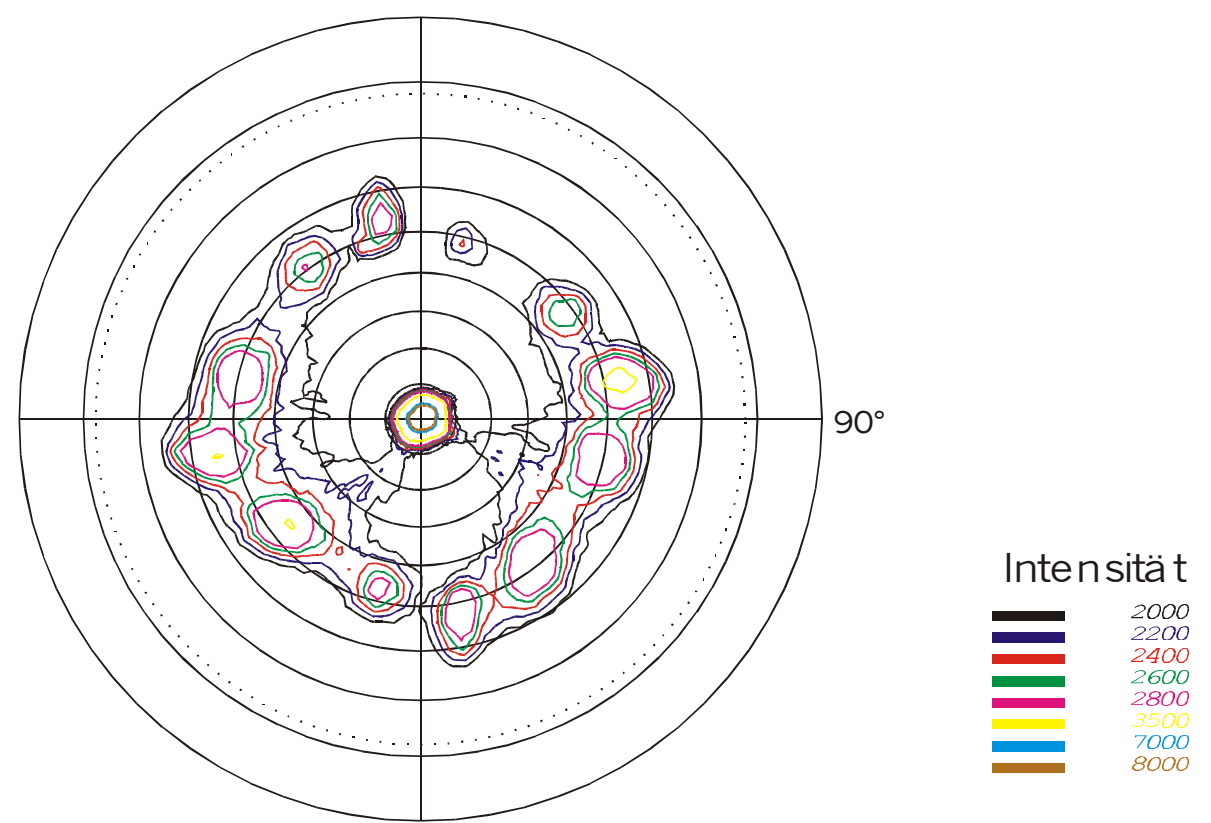

Abb. 4.6 Polfigur des NiO(200)-Reflexes eines unter Argondurchfluss oxidierten Ni-RABiTS-Bandes. Die Endtemperatur betrug $1250^{\circ} \mathrm{C}$.

Für das kubisch flächenzentrierte $\mathrm{NiO}$ gibt diese Figur die Vorzugsrichtung des (111)orientierten Anteils wieder. Außer dem Maximum in der Mitte sind unter einem Verkippungswinkel von $55^{\circ}$ stets zwölf weitere Reflexe zu finden. Die beobachteten 12 Reflexe lassen sich anhand der Abbildung 4.7 anschaulich erklären. Es gibt nämlich 4 Möglichkeiten, wie sich die $\mathrm{NiO}(111)$-Ebenen auf dem $\mathrm{Ni}(100)$ anordnen können. Alle 4 möglichen Anordnungen sind notwendig, um die Ni(100)-Ebenen lückenlos zu bedecken, $\mathrm{NiO}(111)$ kann also nicht mit einer einzigen Orientierung auf Ni(100) aufwachsen. Jeder dieser 4 Varianten erzeugt nun 3 Reflexe unter $55^{\circ}$ in der $\mathrm{NiO}(200)$ Polfigur. 


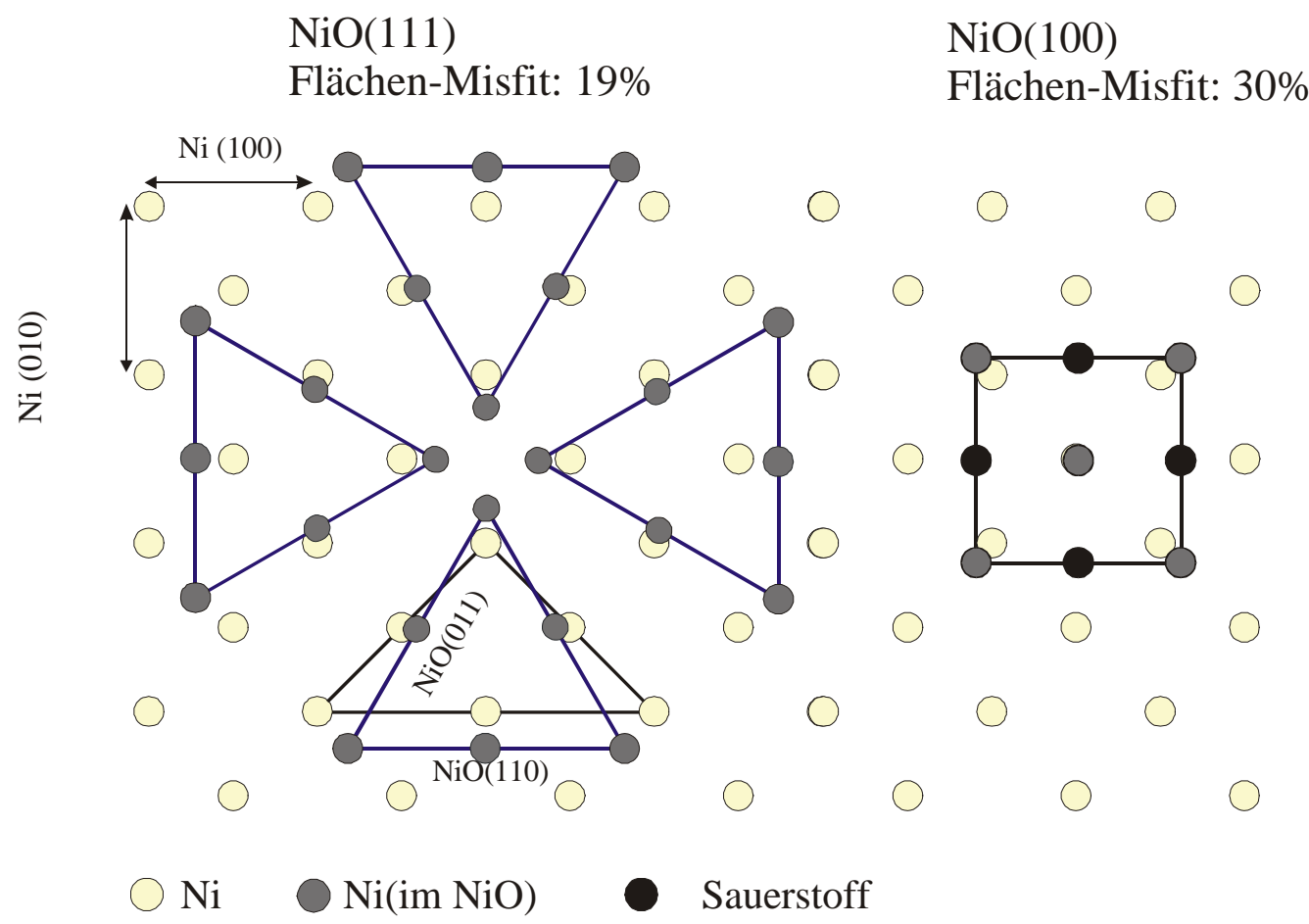

Abb. 4.7 Epitaxie von $\mathrm{NiO}(111)$ und $\mathrm{NiO}(100)$ auf $\mathrm{Ni}(100)$.

Die out-of-plane Texturen des $\mathrm{NiO}(111)$ Anteils liegen zwischen $10^{\circ}$ und $20^{\circ} \mathrm{FWHM}$ und die in-plane Texturen zwischen $11^{\circ}$ und $14^{\circ}$, und sind damit denen des $\mathrm{NiO}(100)$ Anteils sehr ähnlich. Die Gitterfehlpassungen in der Abbildung 4.7 sind als Flächen-Misfits angegeben, d.h. die Oberflächen der Einheitszellen der jeweiligen Orientierung wurden mit der Oberfläche der Einheitszelle des Ni(100) verglichen. Die so erhaltenden Gitterfehlpassungen sind daher ungefähr um einen Faktor 2 größer als die Gitterfehlpassung, die eindimensional angegeben werden. Auffällig ist, dass die Gitterfehlpassung zum NiO(111) viel geringer ist als zum $\mathrm{NiO}(100)$.

\subsection{Der Volumenanteil der Orientierungen}

Sowohl der $\mathrm{NiO}(100)$-Anteil als auch der $\mathrm{NiO}(111)$-Anteil wachsen also epitaktisch auf den $\{100\}<100>$ orientierten Ni, NiCr und NiV-RABiTS-Bänder auf. Soll NiO als Pufferschicht verwendet werden, so muss es eine $\mathrm{NiO}\{100\}<100>$ Textur aufweisen, denn nur auf dieser Textur kann YBaCuO c-Achsen und in-plane-orientiert aufwachsen. In den folgenden zwei Abbildungen 4.8 und 4.9 wird dargestellt, inwieweit der sich bildende $\mathrm{NiO}(100)$-Anteil vom eingestellten Sauerstoffpartialdruck und von der Oxidationstemperatur abhängt. Der Anteil an NiO (100) wurde über das Intensitätsverhältnis des NiO(200)Reflexes und des $\mathrm{NiO}(111)$-Reflexes abgeschätzt.

$\mathrm{I}(100)=\mathrm{I}(200) /((\mathrm{I}(200)+\mathrm{I}(111)) \cdot 100$. 
Da nach Maßgabe der Röntgenmessungen fast ausschließlich $\mathrm{NiO}(111)$ und $\mathrm{NiO}(100)$ auftreten, ergibt sich entsprechend der NiO(111)-Anteil als Differenz aus 100\% und dem $\mathrm{NiO}(100)$-Anteil.

$\mathrm{I}(111)=100-\mathrm{I}(100)$.

Diese Methode zur Bestimmung der Anteile ist nur eine grobe Abschätzung, da die Strukturfaktoren beider Orientierungen nicht gleich groß sind. Im polykristallinen NiO beträgt die Intensität des $\mathrm{NiO}(111)$-Reflexes nur 91\% des $\mathrm{NiO}(200)$-Reflexes. Zudem sind die beiden Anteile nicht immer gleich gut texturiert.

In Abbildung 4.8 ist der so bestimmte $\mathrm{NiO}(100)$-Anteil als Funktion der Oxidationstemperatur dargestellt. Sichtbar ist, dass der NiO(100)-Anteil mit höheren Oxidationstemperaturen bei den verwendeten Ni und NiV RABiTS-Substraten zunimmt. Allerdings ist zu beachten, dass der für die Oxidation verwendete Sauerstoffpartialdruck bei den NiRABiTS-Bänder geringer war als bei den Nickellegierungen. Im Gegensatz zu den oxidierten Ni und NiV-Bändern, bei denen der maximale NiO(100)-Anteil um die 75\% lag, konnte reines $\mathrm{NiO}(100)$-Wachstum bei den oxidierten NiCr-RABiTS-Bändern beobachtet werden, dieses ist im Bereich zwischen $1000^{\circ} \mathrm{C}$ und $1300^{\circ} \mathrm{C}$ temperaturunabhängig. Allerdings nicht unabhängig von dem verwendeten Sauerstoffpartialdruck, wie Abbildung 4.9 zeigt. Der NiO(100)-Anteil fällt mit dem verwendeten Sauerstoffpartialdruck bei den oxidierten Nickellegierungen steigt aber bei reinen Nickel-RABiTS-Bändern.

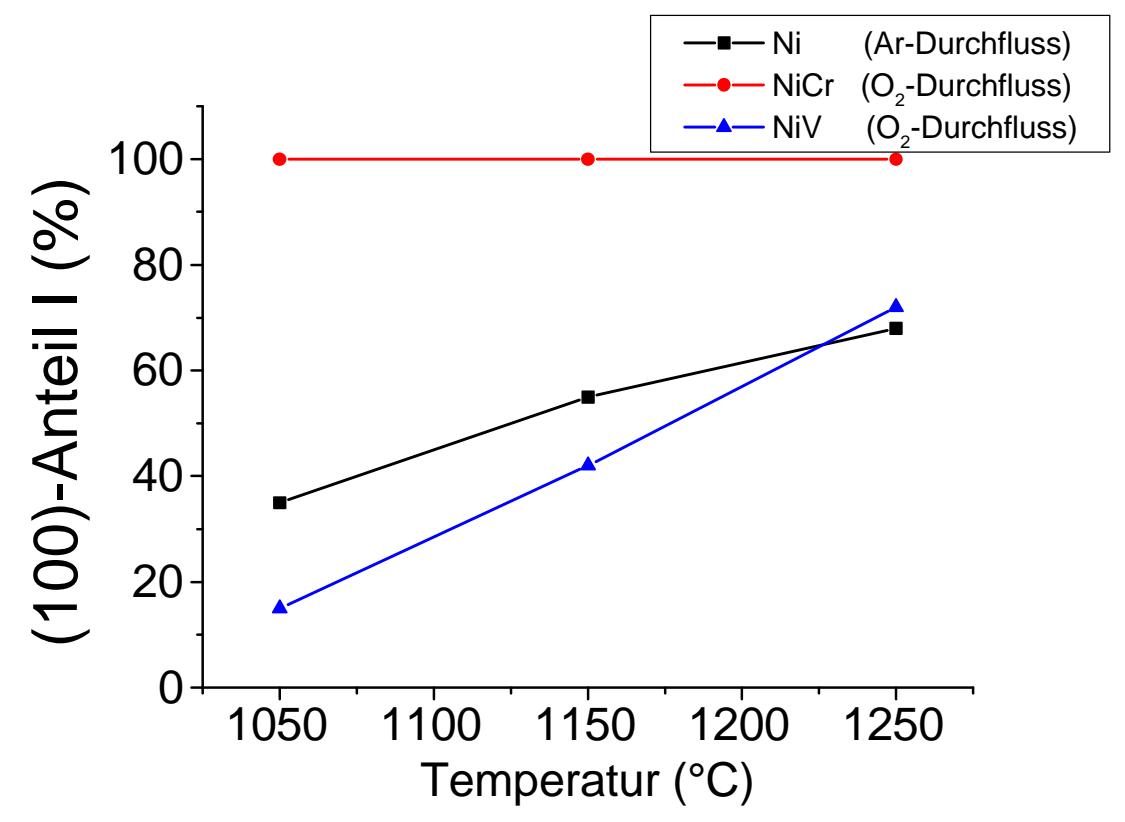

Abb. 4.8 NiO(100)-Anteil in Abhängigkeit von der Oxidationstemperatur und dem RABiTS-Substrat. 


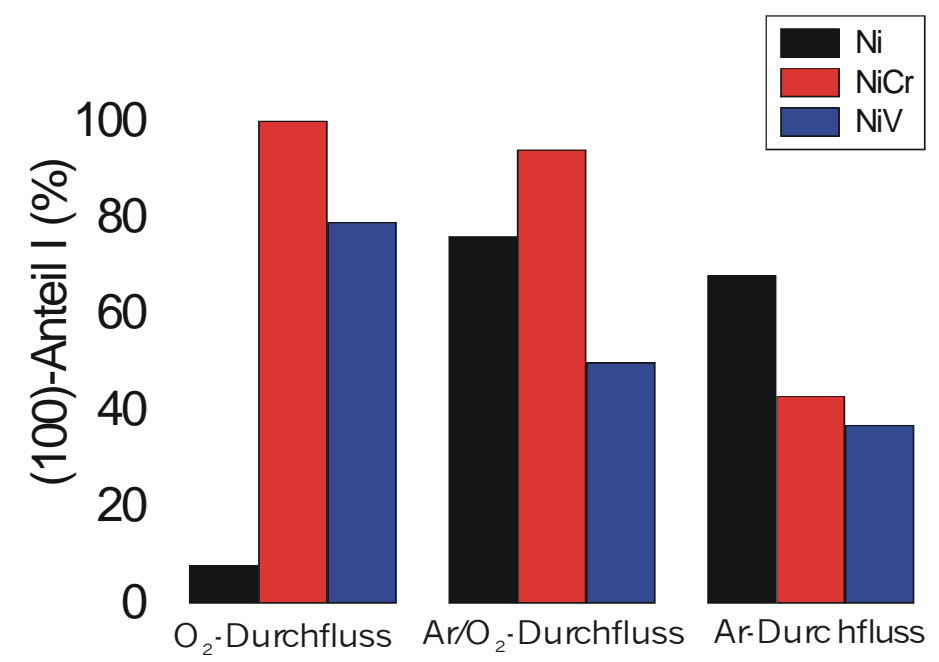

Abb. 4.9 NiO(100)-Anteil in Abhänigkeit vom Sauerstoffpatialdruck und dem RABiTS-Substrat, die Oxidationstemperatur betrug $1250^{\circ} \mathrm{C}$.

\subsection{Die Topologie der Oberflächen}

Alle oxidierten RABiTS-Bänder weisen eine ausgeprägte Oberflächenstruktur und Rauigkeiten, die deutlich über denen der Ausgangsbänder liegen, auf. Die Abbildung 4.10 zeigt die Oberfläche eines mit niedrigem Sauerstoffpartialdruck und $1250^{\circ} \mathrm{C}$ oxidierten reinen $\mathrm{Ni}$-Bandes. Unter diesen Bedingungen weist die Oxidschicht bei reinem Nickel mit ca 70\% den höchsten (100)-Anteil auf. Die Korngrößen variieren zwischen 0,5 und $3 \mu \mathrm{m}$. Die Kornform ist im wesentlichen unregelmäßig, es lassen sich keine Vorzugsorientierungen erkennen. Neben den wenigen eher rechteckigen Körnern lassen sich viele eher dreieckige Strukturen erkennen. Möglicherweise spiegeln diese Strukturen den hohen Anteil der (111)-Orientierung wieder. Die gemittelte Rautiefe $\mathrm{R}_{\mathrm{z}}$ beträgt $1200 \mathrm{~nm}$.

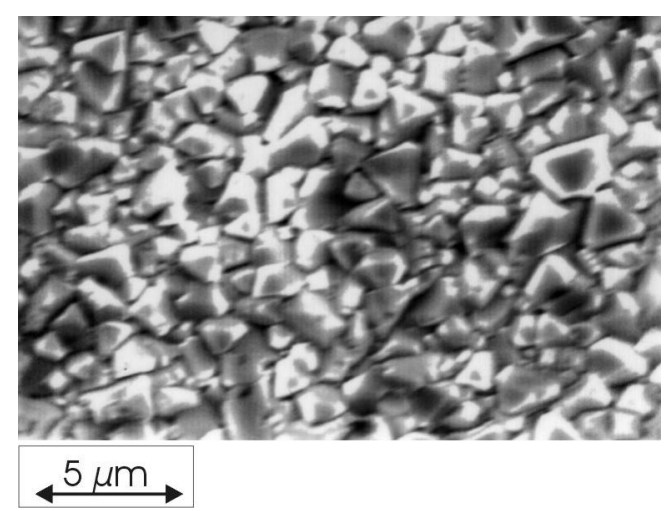

Abb 4.10 REM-Aufnahmen der Oberfläche eines unter Argondurchfluss oxidierten Ni-RABiTSBandes, die Endtemperatur betrug $1250^{\circ} \mathrm{C}$. 
Eine wesentlich kompaktere und gleichförmigere Oberfläche zeigen die NiCr-Proben, die unter höheren Drücken oxidiert wurden und eine reine $\mathrm{NiO}(100)$-Orientierung zeigen (Abb.4.12). Mittels EDX konnten keine Fremdphasen an der Oberfläche gefunden werden. Allerdings bilden sich bei hohen Sauerstoffpartialdrücken und Temperaturen ab $1250^{\circ} \mathrm{C}$ eine Vielzahl von Rissen entlang der kristallographischen (100)-Richtungen des NiO. Sowohl die kristallographische Orientierung als auch der Rissabstand bzw. die Risslänge zeigen an, dass die Rissbildung nicht mit den Korngrenzen des RABiTS-Bandes korreliert sind, sondern allein auf die thermische Spannung während der Oxidation oder während des anschließenden Abkühlens zurückzuführen sind. Die mittlere Rautiefe ist dementsprechend mit $R_{\mathrm{z}}=2400 \mathrm{~nm}$ extrem groß.

Abb. 4.11

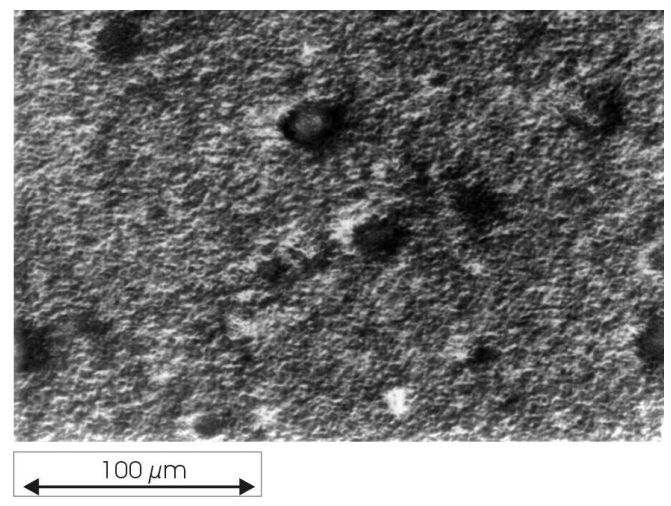

Abb. 4.12

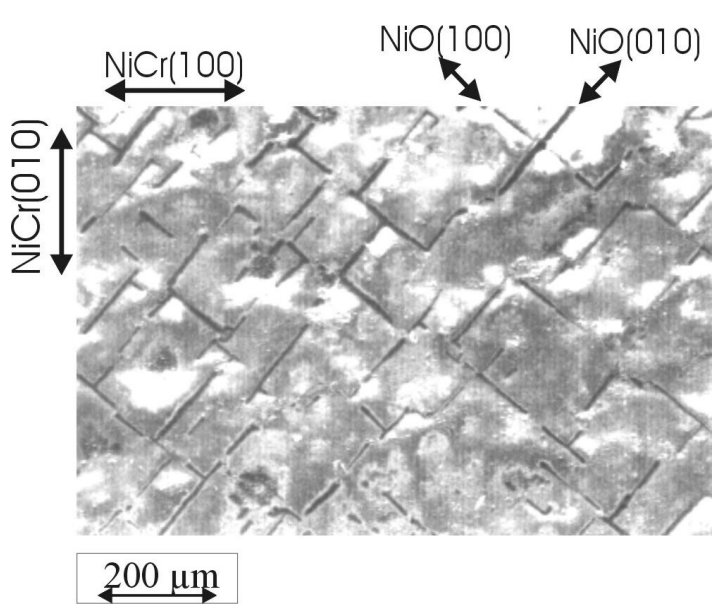

Abb. 4.11 REM-Aufnahmen der Oberfläche eines unter Argondurchfluss oxidierten Ni-RABiTSBandes, die Endtemperatur betrug $1250^{\circ} \mathrm{C}$. Abb. 4.12 REM-Aufnahmen der Oberfläche eines unter Sauerstoffdurchfluss oxidierten NiCr-RABiTS-Bandes, die Endtemperatur betrug $1250^{\circ} \mathrm{C}$.

In den Abbildungen 4.13 bis 4.16 sind die Oberflächen von an Luft oxidierten NiVRABiTS-Bändern dargestellt. Die Oxidationstemperatur wurde zwischen $1050^{\circ} \mathrm{C}$ und $1350^{\circ} \mathrm{C}$ variiert. Auf allen Aufnahmen sind Ausscheidungen zu erkennen, die, außer bei einer Oxidationstemperatur von $1250^{\circ} \mathrm{C}$, bei der sie ellipsenförmig sind, eine nadelförmige Struktur aufweisen. Schichten, die nadelförmige Ausscheidungen enthalten, weisen zusätzlich Risse auf, die jedoch auf den hier gezeigten Aufnahmen nicht sichtbar sind. Da die Risse allerdings nicht von den Ausscheidungen ausgehen, werden auch hier die thermischen Spannungen für die Rissbildung verantwortlich sein. Elementverteilungsbilder zeigen, dass alle Ausscheidungen sehr vanadiumreich und sauerstoffarm sind. Die Größe der Ausscheidungen ist abhängig von der Oxidationstemperatur. Auch die mittlere Rautiefe dieser Schichten ist enorm hoch, sie liegt bei ungefähr $R_{z}=1600 \mathrm{~nm}$. In Tabelle 4.3 sind die Parameter der oxidierten NiV-Bänder dargestellt. Auffallend ist, dass der NiO(100)Anteil gerade dann maximal ist, wenn die vanadiumreichen Auscheidungen ellipsenförmig sind. 
Abb. 4.13

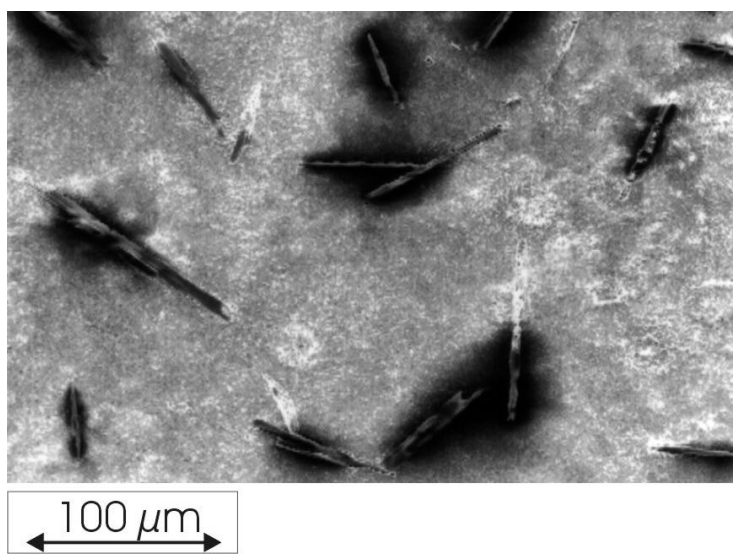

Abb. 4.15

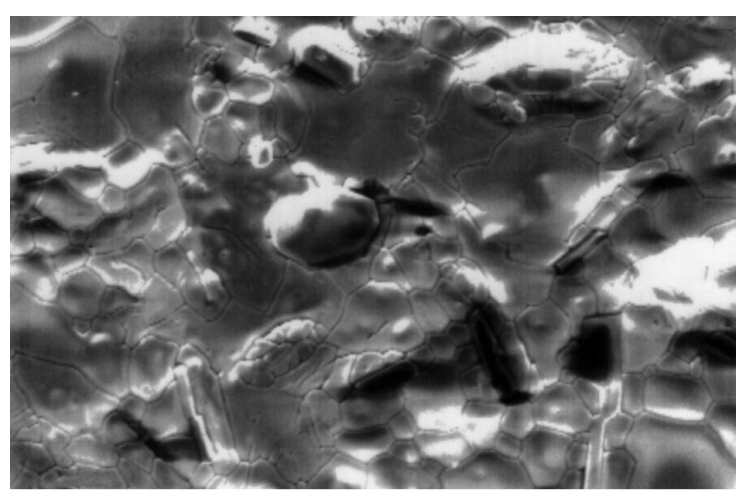

$\stackrel{50 \mu \mathrm{m}}{\longleftarrow}$
Abb. 4.14

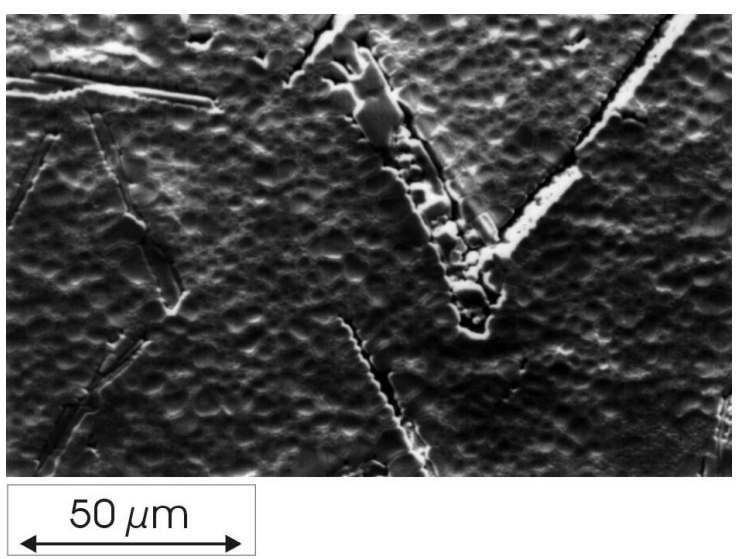

Abb. 4.16

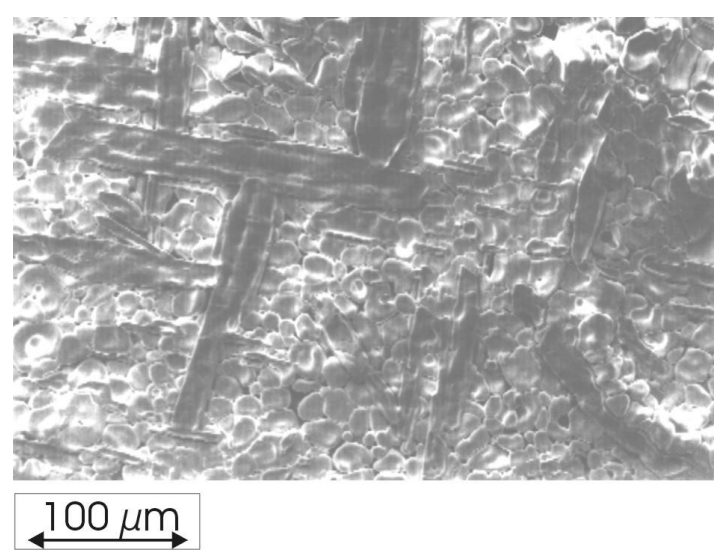

Abb. 4.13-4.16 REM-Aufnahmen von unter Sauerstoffdurchfluss oxidierten NiV-RABiTS- Bändern. Die Oxidationstemperaturen betrugen $1050^{\circ} \mathrm{C}, 1150^{\circ} \mathrm{C}, 1250^{\circ} \mathrm{C}$ und $1350^{\circ} \mathrm{C}$.

\begin{tabular}{|c|c|c|c|c|c|c|}
\hline \multirow[t]{2}{*}{$\begin{array}{l}\text { Oxidations- } \\
\text { temperatur }\left({ }^{\circ} \mathrm{C}\right)\end{array}$} & \multirow[t]{2}{*}{$\begin{array}{l}\mathrm{NiO}(100)- \\
\text { Anteil(\%) }\end{array}$} & \multicolumn{3}{|c|}{$\begin{array}{l}\text { Mittlere } \\
\text { Größe der Ausscheidungen } \\
(\mu \mathrm{m})\end{array}$} & \multirow[t]{2}{*}{ Risse } & \multirow[t]{2}{*}{$\begin{array}{l}\text { Mittlere } \\
\text { Korngröße } \\
(\mu \mathrm{m})\end{array}$} \\
\hline & & Länge & Breite & Form & & \\
\hline 1050 & 15 & $40-100$ & 4-10 & Nadel & ja & 2 \\
\hline 1150 & 42 & $60(10)$ & $4-8$ & Nadel & ja & 4 \\
\hline 1250 & 72 & $30(10)$ & $25(7)$ & Ellipse & nein & 6 \\
\hline 1350 & 32 & $100-150$ & $40(10)$ & Nadel & ja & 12 \\
\hline
\end{tabular}

Tabelle 4.3 Parameter der an Luft oxidierten NiV-RABiTS-Bänder 


\subsection{Einfluss der Substratrauigkeit auf die Oxidrauigkeit}

Die hohen Rauigkeiten der Oxidfilme verhindern jegliche weitere epitaktische Abscheidung, deshalb wird im folgenden untersucht, ob sich durch unterschiedliche Arten von Politur die Oxidrauigkeit minimieren lässt. Dazu werden Ni-RABiTS-Bänder mechanisch und elektrolytisch poliert. Außerdem wird ein sehr glatter epitaktisch auf $\mathrm{SrTiO}_{3}$ abgeschiedener einkristalliner $\{100\}<100>$ Nickelfilm oxidiert. Die Herstellung und Charakterisierung der einkristallinen Filme wird im Kapitel 5.1 genauer beschrieben. In der Tabelle 4.4 sind die Parameter der oxidierten Proben dargestellt.

\begin{tabular}{|c|c|c|c|c|c|c|c|}
\hline & $\begin{array}{l}\text { Mittlere } \\
\text { Rautiefe } \\
\mathrm{R}_{\mathrm{z}} \text { des } \\
\text { Substrats } \\
(\mathrm{nm})\end{array}$ & $\begin{array}{l}\text { Mittlere } \\
\text { Rautiefe } \\
\mathrm{R}_{\mathrm{z}} \text { des } \\
\text { Oxids } \\
(\mathrm{nm})\end{array}$ & $\begin{array}{l}\mathrm{NiO}(100) \\
\text { Anteil(\%) }\end{array}$ & $\begin{array}{l}\text { Out-of-plane } \\
\text { Textur } \\
\text { (100)-Anteil } \\
\text { (FWHM) }\end{array}$ & $\begin{array}{l}\text { In-plane } \\
\text { Textur } \\
\text { (100)-Anteil } \\
\text { (FWHM) }\end{array}$ & $\begin{array}{l}\text { Out-of-plane } \\
\text { Textur } \\
\text { (111)-Anteil } \\
\text { (FWHM) }\end{array}$ & $\begin{array}{l}\text { In-plane } \\
\text { Textur (111)- } \\
\text { Anteil } \\
\text { (FWHM) }\end{array}$ \\
\hline $\begin{array}{l}\text { Einkristalliner } \\
\text { Ni-Film }\end{array}$ & $<20$ & 240 & 78 & 4 & 4 & 7 & 8 \\
\hline $\begin{array}{l}\text { Ni-RABiTS } \\
\text { mechanisch } \\
\text { poliert }\end{array}$ & 80 & 320 & 66 & 10 & 14 & 14 & - \\
\hline $\begin{array}{l}\text { Ni-RABiTS } \\
\text { elektrolytisch } \\
\text { poliert }\end{array}$ & 160 & 390 & 59 & 9 & 14 & 14 & - \\
\hline $\begin{array}{l}\text { Ni-RABiT nicht } \\
\text { poliert }\end{array}$ & 460 & 760 & 28 & 10 & - & 10 & 11 \\
\hline
\end{tabular}

Tab 4.4 Parameter des oxidierten Ni-Films und der Ni-RABiTS-Bänder mit unterschiedlichen Polituren, die Oxidationstemperatur betrug $400^{\circ} \mathrm{C}$, der Druck $1 \cdot 10^{-3}$ mbar $\mathrm{O}_{2}$ und die Zeit 3 Stunden.

In Abbildung 4.17 ist die Oxidrauigkeit $\left(\mathrm{R}_{\mathrm{z}}\right)$ gegen die Substratrauigkeit $\left(\mathrm{R}_{\mathrm{z}}\right)$ aufgetragen. Zwischen Oxid- und Substratrauigkeit besteht ein linearer Zusammenhang, wobei die Steigung ungefähr 1 beträgt. $\left(\mathrm{R}_{\mathrm{z}}^{\mathrm{NiO}}=(216 \pm 9 \mathrm{~nm})+(1,18 \pm 0,04) \mathrm{R}_{\mathrm{z}}^{\mathrm{Ni}}\right)$. Die Substratrauigkeit hat also einen signifikanten Einfluss. Entscheidender ist aber der zweite Beitrag, die allein durch die Oxidation hervorgerufene Rauigkeit, die selbst bei diesen moderaten Oxidationsbedingungen $220 \mathrm{~nm}$ beträgt. Interessant ist auch, dass die Differenz aus Oxid- und Substratrauigkeit nahezu konstant ist. Dies lässt darauf schließen, dass die Rauigkeitsentwicklung unabhängig vom Substrat immer dieselbe ist. Diese Rauigkeitsentwicklung wird im Kapitel 5.4 näher untersucht. Bemerkenswerterweise nimmt der $\mathrm{NiO}(100)$-Anteil linear mit zunehmender Substratrauigkeit ab. Hinreichend glatte Substrate sind also nicht nur zur Reduktion der Oxidrauigkeit sondern auch zur Vermeidung von Fehlorientierungen notwendig. 


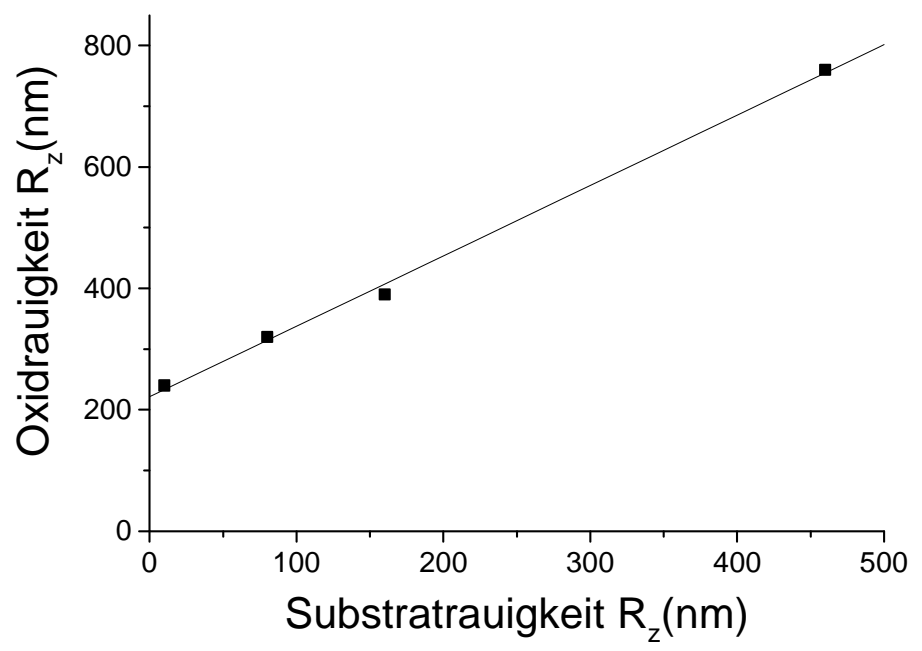

Abb 4.17 Mittlere Rautiefe $R_{z}$ des Oxides aufgetragen gegen die mittlere Rautiefe des Substrates bei konstanten Oxidationsbedingungen.

\subsection{Schlussfolgerung}

Mittels einfacher experimenteller Methoden ist es möglich $\mathrm{Ni}$, NiCr und NiV-RABiTSBänder so oxidieren zu lassen, dass sich das Eigenoxid epitaktisch bildet. Allerdings bildet sich nicht nur die gewünschte $\mathrm{NiO}(100)$-Textur, sondern ebenfalls eine $\mathrm{NiO}(111)$-Textur. Durch geeignete Wahl der Oxidationsparameter kann die unerwünschte $\mathrm{NiO}(111)$-Textur bei der Oxidation von NiCr-RABiTS-Bändern vollständig unterdrückt werden, wobei bei der Oxidation von Ni und NiV-RABiTS-Bändern immer ein kleiner $\mathrm{NiO}(111)$-Anteil beobachtet wurde. Der $\mathrm{NiO}(100)$-Anteil kann innerhalb der Ebene in der $0^{\circ}$ oder der $45^{\circ}$ Epitaxiebeziehung aufwachsen. Die Relation lässt sich über den Sauerstoffpartialdruck einstellen. Die Oberflächen der oxidierten Ni und NiCr-Bänder bestehen aussschließlich aus $\mathrm{NiO}$, die Oberflächen der oxidierten NiV-Bänder zeigen zusätzlich vanadiumreiche Ausscheidungen. Von den hochtemperaturoxidierten Bändern sind also nur NiCr und eingeschränkt Ni als Puffer geeignet. Allerdings verhindern hier die großen Rauigkeiten die epitaktische Abscheidung weiterer Puffer-oder HTSL-Schichten. Die Textur- und Rauigkeitsentwicklung wird im folgenden Kapitel auf einkristallinen Modellsubstraten eingehend untersucht. 


\section{Epitaktische Oxidation einkristalliner Ni und NiCr-Filme}

Um die Mechanismen der epitaktischen Oxidation genauer zu verstehen, werden die folgenden Experimente an epitaktisch auf einkristallinen Substraten abgeschiedenen Filmen durchgeführt. Diese Filme werden im folgenden zur Unterscheidung von RABiTS-Bändern als einkristalline Filme bezeichnet. Außerdem werden die Oxidationsexperimente unter sehr definierten Sauerstoffpartialdrücken und Temperaturen durchgeführt.

\subsection{Charakterisierung der einkristallinen Filme}

Die einkristallinen $\mathrm{Ni}$ und $\mathrm{Ni}_{87} \mathrm{Cr}_{13}$-Filme wurden mittels Ionenstrahlsputterns bei $300^{\circ} \mathrm{C}$ epitaktisch auf $\mathrm{SrTiO}_{3}(100) \mathrm{K}$-Einkristallen abgeschieden. Röntgenmessungen zeigen, dass die so hergestellten $\{100\}<100>$ orientierten Ni-Filme out-of-plane und in-plane Texturen von unter $0,7^{\circ}$ Halbwertsbreite aufweisen. Die abgeschiedenen Nickelfilme sind sehr glatt, die Mittenrauigkeit liegt bei $\mathrm{R}_{\mathrm{a}}=0,4 \mathrm{~nm}$. Die Abb. 5.1 zeigt eine TEM-Aufnahme eines epitaktisch abgeschiedenen Nickelfilms im Querschnitt. An den dazu gezeigten Beugungsbilder ist zu erkennen, dass das Nickel einkristallin und cube-on-cube auf dem $\mathrm{SrTiO}_{3}$ aufgewachsen ist. Der Nickelfilm ist von vielen Stapelfehlern und Versetzungen, deren Dichte $3 \cdot 10^{8} / \mathrm{cm}^{2}$ beträgt, durchsetzt. Die auf den Nickelfilm aufgebrachte Goldschicht dient hier nur als Markerschicht für die Oberfläche des Nickels. Im Gegensatz zum quasieinkristallinen Nickelfilm zeigt der $\mathrm{Ni}_{87} \mathrm{Cr}_{13}$-Film säulenartiges Wachstum. Die Dunkelfeldaufnahme des NiCr-Filmes (Abb. 5.2) wurde auf dem (001)-Reflex aufgenommen. Die dunklen Bereiche der Säulen bestehen aus Körnern, die um $1^{\circ}-10^{\circ}$ aus der (001)-Richtung herausgekippt sind. Da sowohl die Rockingkurven als auch der $\phi$-Scan dieses Filmes Halbwertsbreiten von weniger als 1,3 $3^{\circ}$ FWHM aufweisen, ist allerdings davon auszugehen, dass der Volumenanteil der um mehrere Grad verkippten Körner sehr gering ist. Die Beugungsbilder weisen auch bei diesem Schichtsystem epitaktisches bzw. stark vorzugsorientiertes Wachstum nach. Da die Gitterfehlpassungen zwischen $\mathrm{SrTiO}_{3}$ und $\mathrm{Ni}$, und $\mathrm{SrTiO}_{3}$ und $\mathrm{Ni}_{87} \mathrm{Cr}_{13}$ fast identisch sind, ist das Auftreten des säulenartigen Wachstums nicht auf eine veränderte Gitterfehlpassung zurückzuführen. 


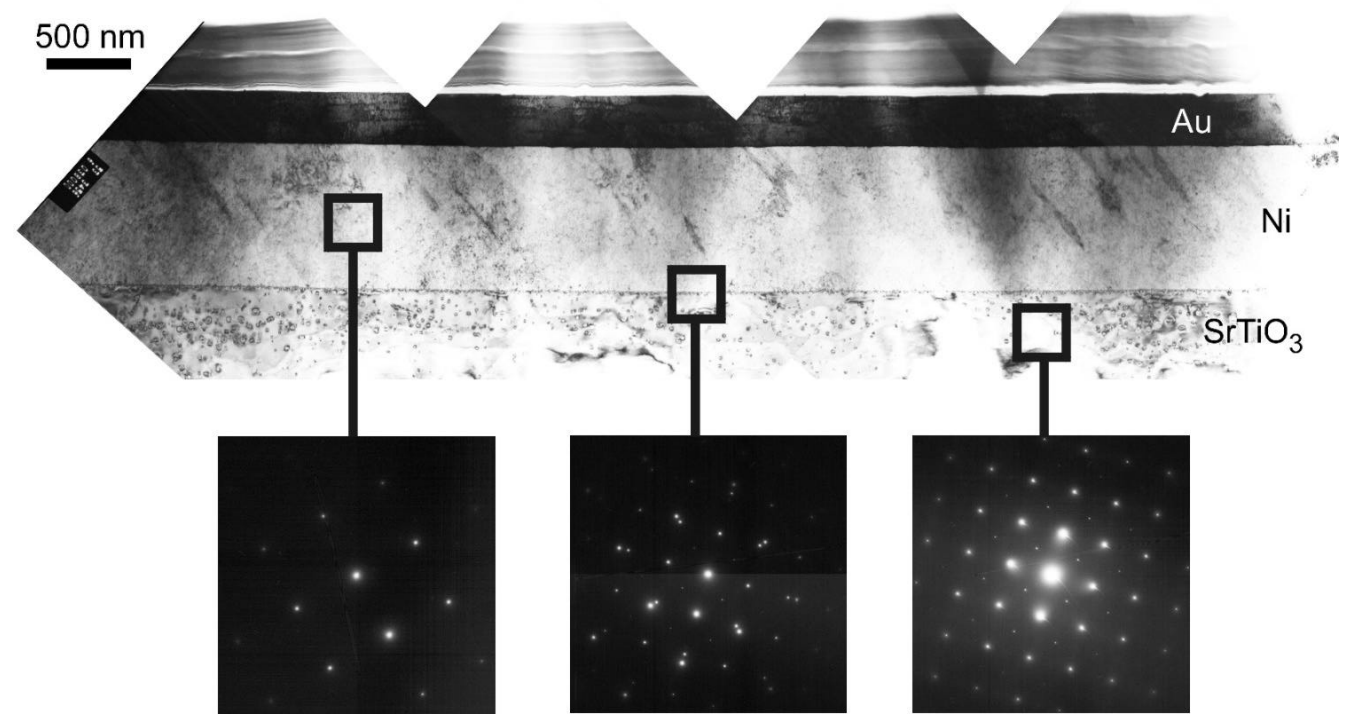

Abb. 5.1 TEM-Aufnahme eines auf $\mathrm{SrTiO}_{3}$ abgeschiedenen Ni-Films im Querschnitt.
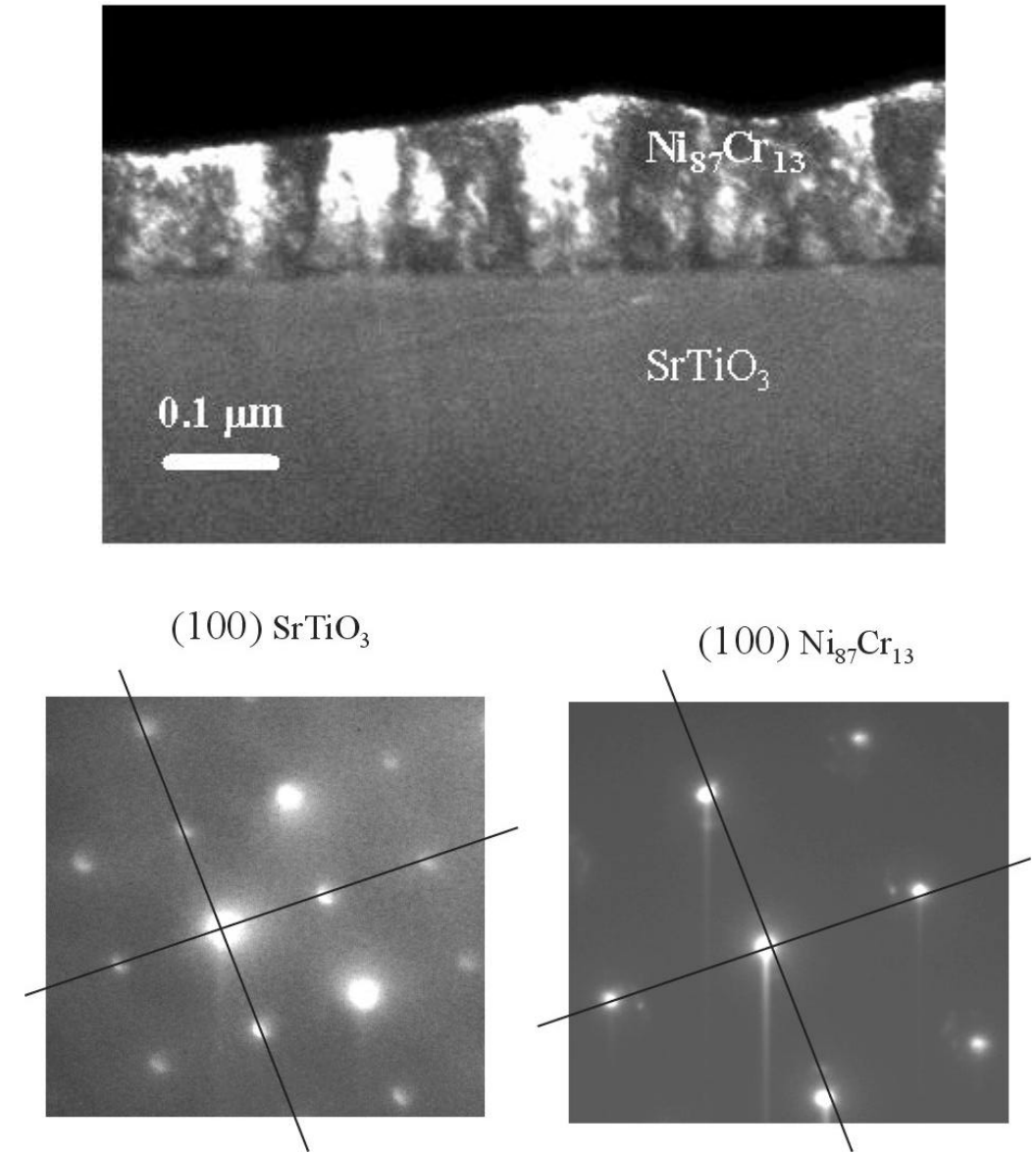

Abb. 5.2 TEM-Aufnahme eines auf $\mathrm{SrTiO}_{3}$ abgeschiedenen $\mathrm{Ni}_{87} \mathrm{Cr}_{13}$ Films im Querschnitt. 


\subsection{Vorzugsorientierungen in kompakten NiO-Filmen}

\subsubsection{Oxidation der einkristallinen Ni-Filme}

Die einkristallinen Nickelfilme wurden sowohl in einer Hochvakuumanlage als auch insitu in einer in einem 2-Kreis-Diffraktometer integrierten Oxidationskammer, so dass das Wachstum der Oxidschicht während der Oxidation beobachtet werden konnte, oxidiert. Während der Aufheiz-und Abkühlphase befand sich die Nickelschicht im Vakuum, das in der Hochvakuumanlage besser als $5 \cdot 10^{-6}$ mbar und im 2-Kreis-Diffraktometer besser als $1 \cdot 10^{-5}$ mbar war. Die Röntgendiffraktogramme der oxidierten Schichten zeigten $\mathrm{NiO}(111)$ und $\mathrm{NiO}(200)$-Reflexe. Andere $\mathrm{NiO}$ Reflexe wie z.B. der $\mathrm{NiO}(220)$-Reflex wurden nicht beobachtet. Rockingkurven und $\phi$-Scans zeigten, dass beide Anteile, sowohl der NiO(100)Anteil als auch der $\mathrm{NiO}(111)$-Anteil, eine out-of-plane und eine in-plane Textur besitzen. Der Anteil an NiO (100) wurde hier wieder wie bereits im vorigen Kapitel (4.3) beschrieben über das Intensitätsverhältnis des $\mathrm{NiO}(200)$-Reflexes und des $\mathrm{NiO}(111)$-Reflexes abgeschätzt.

Die Ergebnisse der Oxidationsexperimente, die in der Hochvakuumanlage durchgeführt worden sind, sind in der Abbildung 5.3 zusammengefasst. Die Zahlen in dem DruckTemperatur- Diagramm geben den NiO(100)-Anteil in Prozent an.

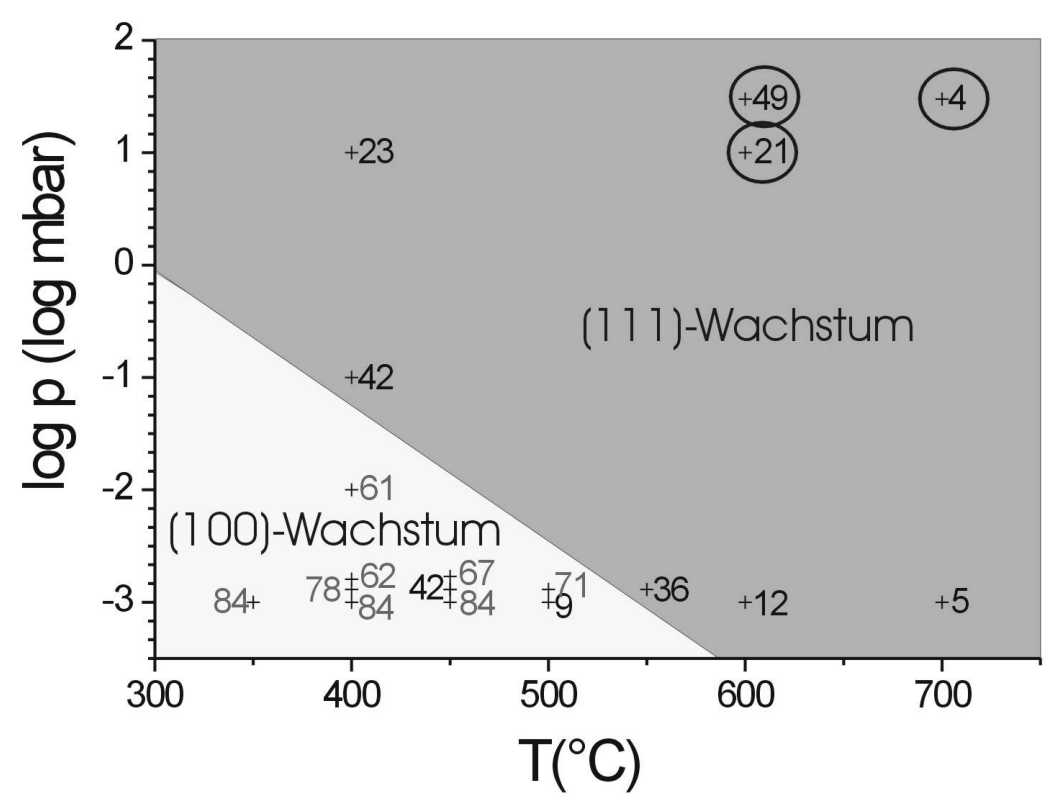

Abb. 5.3 NiO(100)-Anteil in Abhängigkeit von der Oxidationstemperatur und dem Sauerstoffpartialdruck. Die Zahlen geben den NiO(100)-Anteil in Prozent an. Der NiO(111)-Anteil ist die Differenz aus $\mathbf{1 0 0} \%$ und dem NiO(100)-Anteil. Die Oxidationszeiten betrugen fast immer 3 Stunden, nur die der eingekreisten Messpunkte betrugen 30 Minuten. Die zeitliche Entwicklung der Anteile wird im Kapitel 5.3 dargestellt. 
Die Streuung des $\mathrm{NiO}(100)$-Anteils ist relativ groß, dennoch ist der generelle Trend zu erkennen, dass bei niedrigen Temperaturen und Sauerstoffpartialdrücken der NiO(100)Anteil überwiegt und bei hohen Temperaturen und hohen Sauerstoffparitaldrücken der NiO(111)-Anteil. Die scharfe Abgrenzung der beiden Wachstumsbereiche in der Grafik wurde nur zur besseren Übersicht eingezeichnet. Die out-of-plane Texturen des NiO(100)Anteils liegen zwischen $8^{\circ}$ und $16^{\circ}$ FWHM und die in-plane Texturen liegen zwischen $5^{\circ}$ und $10^{\circ} \mathrm{FWHM}$, wobei der $\mathrm{NiO}(100)$ Anteil in-plane immer cube-on-cube auf dem einkristallinen Nickelfilm aufgewachsen ist. Meistens sind die in-plane Texturen besser als die out-of-plane Texturen. Ebenso sind auch die out-of-plane Texturen des $\mathrm{NiO}(111)$, die zwischen $3^{\circ}$ und $11^{\circ} \mathrm{FWHM}$ liegen, häufig schlechter als die in-plane Texturen, die zwischen $5^{\circ}$ und $9^{\circ}$ liegen. Wie bei der Oxidation der RABiTS-Bänder wächst auch hier der $\mathrm{NiO}(111)$-Anteil in-plane in vier verschiedenen um jeweils $90^{\circ}$ gedrehten Orientierungen auf (vgl. Abb. 4.7).

Die gleiche Tendenz findet man bei Oxidationen in der im Röntgendiffraktometer integrierten Vakuumkammer, nämlich hohe $\mathrm{NiO}(100)$-Anteile bei niedrigen Temperaturen und Sauerstoffpartialdrücken und hohe $\mathrm{NiO}(111)$-Anteile bei hohen Temperaturen und Sauerstoffpartialdrücken. Allerdings ist der Bereich des $\mathrm{NiO}(100)$ zu höheren Temperaturen (ca. $100^{\circ} \mathrm{C}$ ) verschoben. Dies kann zum einen an der Temperaturmessung liegen und zum anderen daran, dass die Ni-Filme für die Messungen in der Röntgenapparatur mit einem anderen Ni-Target hergestellt wurden, denn geringe Verunreinigungen und Oberflächen-AdAtome können den Oxidationsmechanismus erheblich beeinflussen. Auf diesen Punkt und die generelle schwierige genaue Reproduzierbarkeit wird im Kapitel 6.3.5 näher eingegangen.

\subsubsection{Oxidation einkristalliner $\mathrm{Ni}_{87} \mathrm{Cr}_{13}$-Filme}

Die einkristallinen NiCr-Filme wurden ebenfalls in einer Hochvakuumanlage bei unterschiedlichen Temperaturen und Sauerstoffpartialdrücken oxidiert. Allerdings mußten hierbei höhere Temperaturen und Sauerstoffpartialdrücke gewählt werden als bei der Oxidation der reinen Nickelfilme, da bei niedrigeren Temperaturen und Sauerstoffpartialdrücken keine NiO-Reflexe beobachtet wurden. Vermutlich bildet sich Chromoxid, diese Schicht muß aber sehr dünn sein, da keine Chromoxidreflexe im Röntgendiffraktogramm beobachtet wurden. Da für die Oxidation hohe Temperaturen und Sauerstoffpartialdrücke verwendet wurden, konnten keine in-situ Experimente in der Röntgenapparatur durchgeführt werden, da der Rezipient des $\Theta-2 \Theta$-Diffraktometers nicht für diese Parameter ausgelegt ist. 
In Abbildung 5.4a,b ist der $\mathrm{NiO}(100)$-Anteil gegen die Oxidationstemperatur bzw. gegen den Sauerstoffpartialdruck aufgetragen

a)

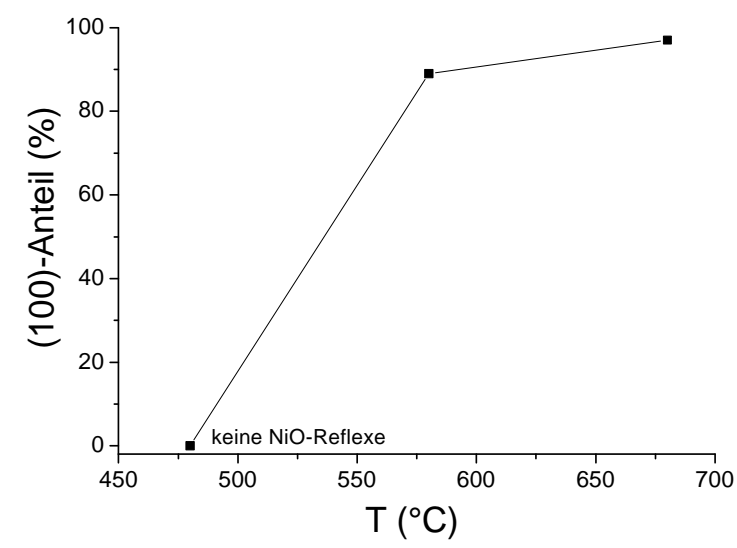

b)

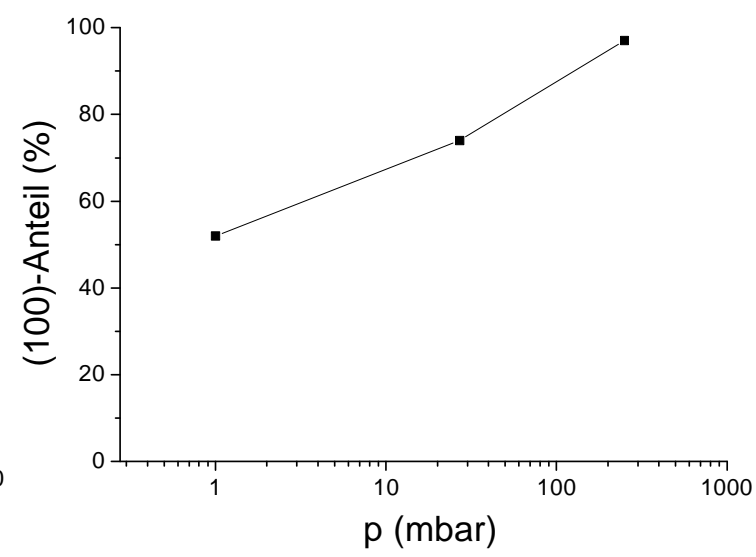

Abb. 5.4 a) NiO(100)-Anteil in Abhängigkeit von der Oxidationstemperatur, der Sauerstoffpartialdruck betrug 250 mbar $\mathrm{O}_{2}$ und die Oxidationszeit betrug 30 Minuten. b) NiO(100)-Anteil in Abhängigkeit vom Sauerstoffpartialdruck, die Oxidationstemperatur betrug $680^{\circ} \mathrm{C}$ und die Zeit 30 Minuten.

Im Gegensatz zu der Oxidation des reinen Nickelfilms nimmt der $\mathrm{NiO}(100)$-Anteil bei der Oxidation des NiCr-Films mit steigender Temperatur und steigendem Sauerstoffpartialdruck zu. Unterhalb einer Temperatur von $550^{\circ} \mathrm{C}$ wurden keine NiO-Reflexe im Röntgendiffraktogramm beobachtet. Die out-of-plane und in-plane Texturen des $\mathrm{NiO}(111)$ und $\mathrm{NiO}(100)$-Anteil wurden mittels Rockingkurve und $\phi$-Scan bestimmt und sind in Tabelle 5.1 dargestellt.

\begin{tabular}{|c|c|c|c|c|c|c|l|}
\hline $\begin{array}{c}\text { Tempe- } \\
\text { ratur }\left({ }^{\circ} \mathrm{C}\right)\end{array}$ & $\begin{array}{l}\text { Druck } \\
(\mathrm{mbar})\end{array}$ & $\begin{array}{l}\text { NiO(100)- } \\
\text { Anteil (\%) }\end{array}$ & $\begin{array}{l}\text { Out-of-plane } \\
\text { Textur(100) } \\
(\mathrm{FWHM})\end{array}$ & $\begin{array}{l}\text { In-plane } \\
\text { Textur(100) } \\
(\mathrm{FWHM})\end{array}$ & $\begin{array}{l}\text { NiO(111)- } \\
\text { Anteil (\%) }\end{array}$ & $\begin{array}{l}\text { Out-of-plane } \\
\text { Textur(111) } \\
(\mathrm{FWHM})\end{array}$ & $\begin{array}{l}\text { In-plane } \\
\text { Textur(111) } \\
(\mathrm{FWHM})\end{array}$ \\
\hline 580 & 250 & 89 & 3 & 3,2 & 11 & - & - \\
\hline 680 & 250 & 97 & 1,6 & 2,0 & 3 & - & - \\
\hline 680 & 1 & 52 & 1,7 & 2,4 & 48 & 0,6 & 4,5 \\
\hline 680 & 27 & 74 & 1,6 & 1,7 & 26 & 4,6 & 4,2 \\
\hline
\end{tabular}

Tab. 5.1 Texturen des NiO der oxidierten einkristallinen NiCr-Filme.

Sowohl die out-of-plane als auch die in-plane Texturen sind wesentlich besser als die der oxidierten Nickelfilme. Im Gegensatz zu den oxidierten NiCr-RABiTS-Bändern wurden bei den oxidierten einkristallinen NiCr-Filmen keine $\mathrm{NiCr}_{2} \mathrm{O}_{4}$ und $\mathrm{Cr}_{2} \mathrm{O}_{3}$-Reflexe im 
$\Theta-2 \Theta-$ Röntgendiffraktogramm beobachtet. Allerdings zeigt sich hier ebenso der Trend, dass mit steigendem Sauerstoffpartialdruck der NiO(100)-Anteil zunimmt.

Die vorgestellten Ergebnisse beschreiben die Texturverteilung, die röntgenographisch nach Abschluss der Oxidation zu beobachten ist. Die folgenden Experimente werden durchgeführt, um zu untersuchen ob und gegebenenfalls wie sich die Textur der aufwachsenden Oxidschicht im Verlauf der Oxidwachstums entwickelt.

\subsection{Texturentwicklung und Kinetik der Oxidation einkristalli- ner Nickelfilme}

\subsubsection{Texturentwicklung in dicken NiO-Schichten}

Im Röntgendiffraktometer wurde der $\mathrm{NiO}(100)$-Anteil während der Oxidation einkristalliner Nickelfilme gemessen, indem alle $80 \mathrm{~s}$ ein $\Theta-2 \Theta$-Diffraktogramm aufgenommen wurde. In den Abbildungen 5.5a,b ist der $\mathrm{NiO}(100)$ Anteil gegen die Oxidationszeit aufgetragen. Die Oxidationstemperatur betrug $500^{\circ} \mathrm{C}$ bzw. $680^{\circ} \mathrm{C}$.

a)

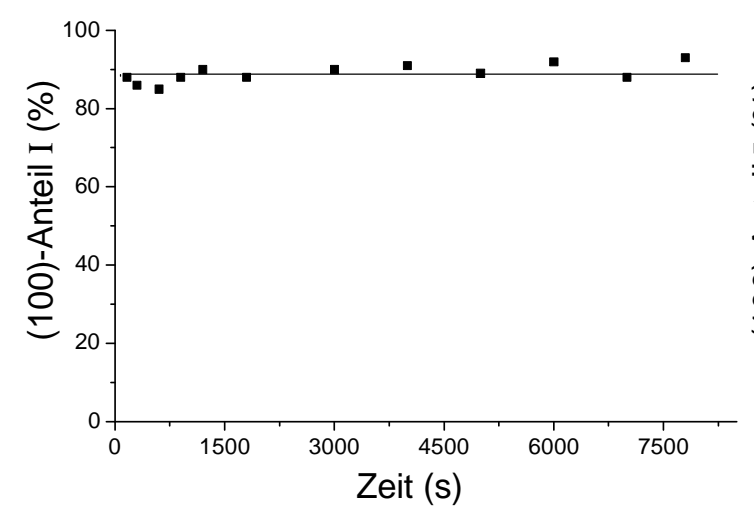

b)

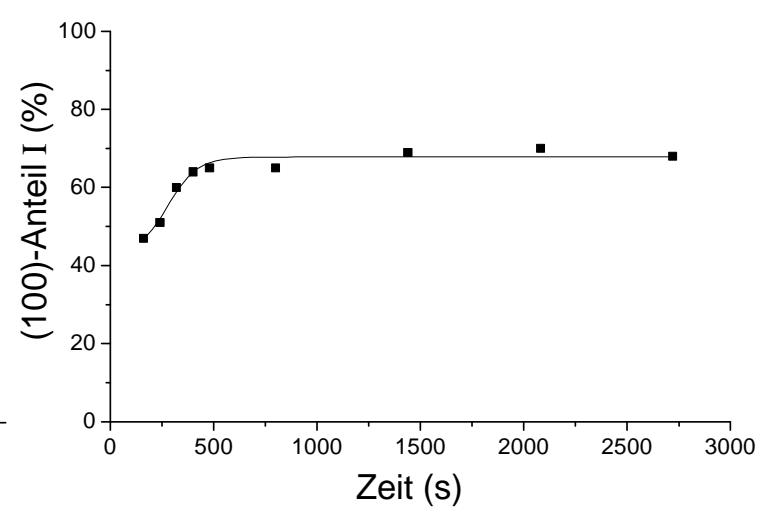

Abb. 5.5 a) $\mathrm{NiO(100)-Anteil} \mathrm{in} \mathrm{Abhängigkeit} \mathrm{von} \mathrm{der} \mathrm{Oxidationszeit,} \mathrm{die} \mathrm{Temperatur} \mathrm{betrug} 500^{\circ} \mathrm{C}$, der Sauerstoffpartialdruck $2 \cdot 10^{-3}$ mbar $\mathrm{O}_{2}$.

b) NiO(100)-Anteil in Abhängigkeit von der Oxidationszeit, die Temperatur betrug $680^{\circ} \mathrm{C}$, der Sauerstoffpartialdruck $2 \cdot 10^{-3}$ mbar $\mathrm{O}_{2}$.

Diese Messungen zeigen exemplarisch für viele andere, dass nach wenigen Minuten der Oxidation der $\mathrm{NiO}(100)$ bzw. $\mathrm{NiO}(111)$-Anteil konstant bleiben. Bei höheren Temperaturen oder Sauerstoffpartialdrücken steigt der $\mathrm{NiO}(100)$ Anteil in den ersten Minuten der Oxidation stark an. Dagegen ist bei bei niedrigen Temperaturen und Sauerstoffpartialdrukken der NiO(100)-Anteil von Beginn der Messung an konstant. Allerdings können mit der Röntgenapparatur und den hier verwendeten Messzeiten Röntgenreflexe erst ab einer Schichtdicke von ca. 30-50 nm beobachtet werden. Aussagen über das Anfangstadium der 
Oxidation können mit dieser Messmethode nicht gemacht werden. Um zu überprüfen, ob es eine Texturentwicklung während der Oxidation gibt, wurden weiterhin Rockingkurven aufgenommen. In Abbildung 5.6 sind deren Halbwertsbreiten gegen die Oxidationszeit aufgetragen.

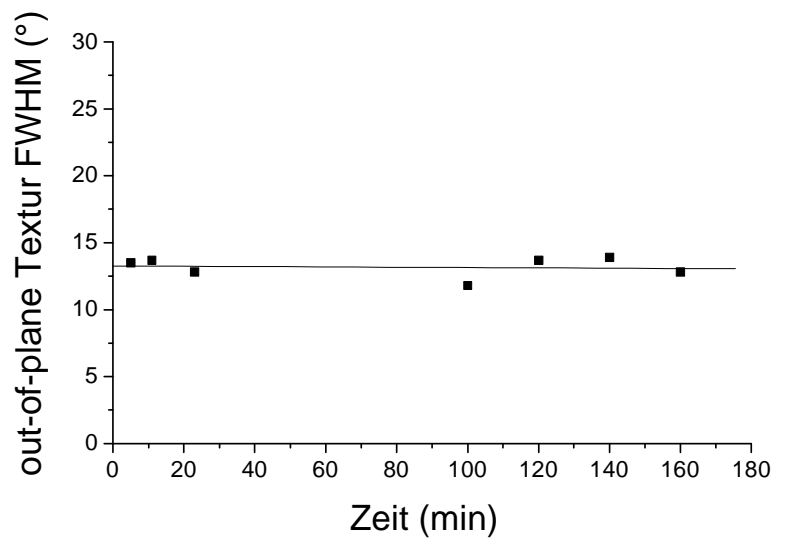

Abb. 5.6 Halbwertsbreiten der NiO(200)-Rockingkurven in Abhängigkeit von der Oxidationszeit. Die Oxidationstemperatur betrug $580^{\circ} \mathrm{C}$, der Sauerstoffpartialdruck $2 \cdot 10^{-3}$ mbar $\mathrm{O}_{2}$.

Wie die Abbildung 5.6 zeigt, gibt es keine Texturentwicklung, sondern lediglich ein epitaktisches Weiterwachsen. Da sich weder der NiO(100)-Anteil noch die out-of-plane Texturen mit der Zeit ändern, steigt die Intensität des $\mathrm{NiO}(200)$-Reflex proportional mit der Dicke der NiO-Schicht an, solange die Schichtdicke viel dünner als die Eindringtiefe des Röntgenstrahls ist. Dies trifft für diese Messungen zu, da die Eindringtiefe des Röntgenstrahls bis zu mehreren Mikrometern reicht, während die Dicke der Oxidschicht durch die Dicke der Ni-Schicht auf maximal $800 \mathrm{~nm}$ begrenzt ist. In Abbildung 5.7 ist die Intensität des $\mathrm{NiO}(200)$-Reflexes gegen die Wurzel der Zeit aufgetragen. Sehr eindeutig ist eine lineare Abhängigkeit zu erkennen. Das Wachstum der NiO-Schicht geschieht also nach einem parabolischen Wachstumsgesetz.

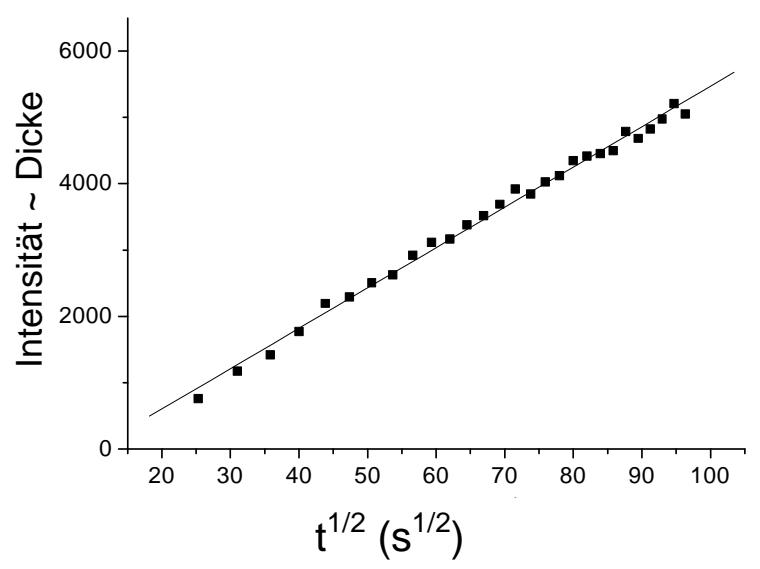

Abb. 5.7 Intensität des NiO(200)-Reflexes in Abhängigkeit von der Wurzel der Oxidationszeit. Die Oxidationstemperatur betrug $500^{\circ} \mathrm{C}$, der Sauerstoffpartialdruck $2 \cdot 10^{-3}$ mbar $\mathrm{O}_{2}$. 
In den folgenden Experimenten soll überprüft werden, inwieweit die Änderung der Oxidationsparameter, Temperatur und Sauerstoffpartialdruck, bei NiO-Schichten, die schon eine gewisse Dicke haben, das Wachstum der beiden NiO-Anteile beeinflusst. Dazu wird in einem Experiment der Sauerstoffpartialdruck nach einstündiger Oxidation um vier GröBenordnungen und in einem anderen die Temperatur nach einer bzw. nach zwei Stunden Oxidation erhöht. Die Abbildungen 5.8a,b zeigen diese Messungen zeitaufgelöst.

a)

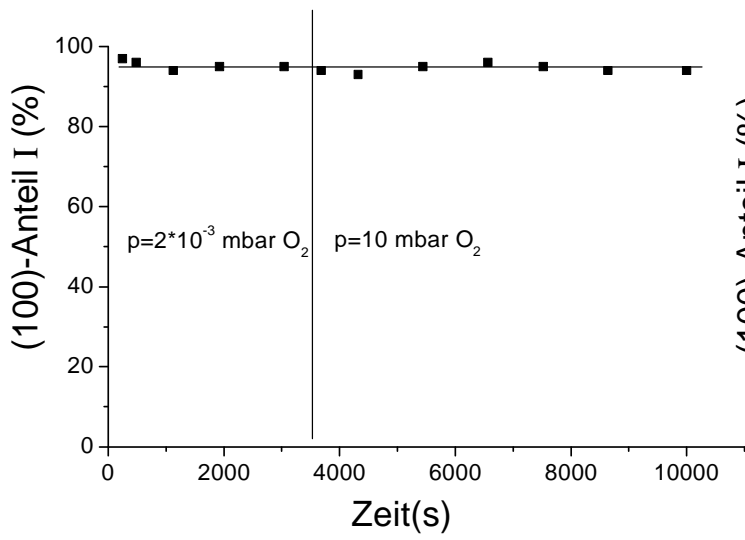

b)

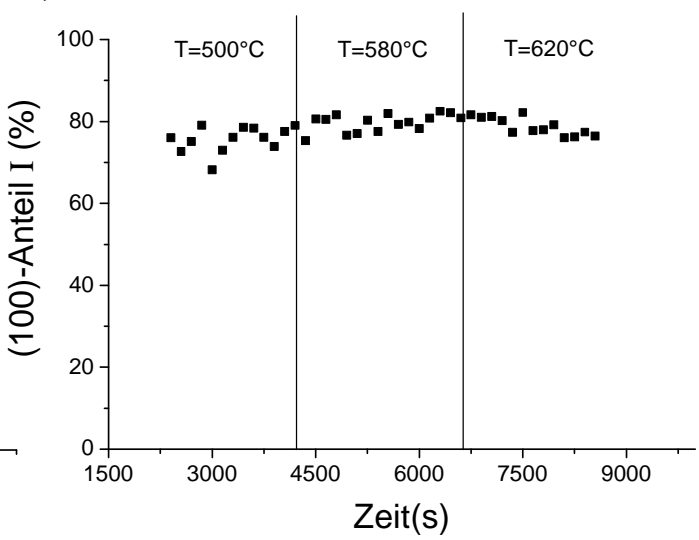

Abb. 5.8 a) $\mathrm{NiO(100)-Anteil} \mathrm{in} \mathrm{Abhängigkeit} \mathrm{von} \mathrm{der} \mathrm{Oxidationszeit.} \mathrm{Die} \mathrm{Temperatur} \mathrm{betrug} 580^{\circ} \mathrm{C}$, der Sauerstoffparialdruck wurde nach einer Stunde von $2 \cdot 10^{-3}$ auf 10 mbar $\mathrm{O}_{2}$ erhöht. b) $\mathrm{NiO}(100)$ Anteil in Abhängigkeit von der Oxidationszeit, der Sauerstoffpartialdruck betrug $2 \cdot 10^{-3}$ mbar $\mathrm{O}_{2}$, die Temperatur wurde nach einer Stunde von $500^{\circ} \mathrm{C}$ auf $580^{\circ} \mathrm{C}$ und nach weiteren 50 Minuten auf $620^{\circ} \mathrm{C}$ erhöht.

Hat die NiO-Schicht bereits eine gewisse Dicke erreicht, so ist das Wachstum der beiden Anteile, $\mathrm{NiO}(111)$ und $\mathrm{NiO}(100)$, gleich schnell, unabhängig von den Oxidationsparametern wachsen die schon vorhanden Anteile im gleichen Verhältnis epitaktisch weiter.

Da die in Abbildung 5.3 dargestellten Messungen aber eindeutig belegen, dass bei der Oxidation bei niedrigen Temperaturen und Sauerstoffpartialdrücken hauptsächlich NiO(100) und bei hohen Temperaturen und Sauerstoffpartialdrücken hauptsächlich $\mathrm{NiO}(111)$ ensteht, folgt zwangsläufig aus den eben gezeigten Texturentwicklungen in dikken NiO-Schichten, dass die Verhältnisse der beiden NiO-Anteile und die Texturen fast ausschließlich von den ersten Minuten der Oxidation abhängen. Deshalb wird in den folgenden Experimenten die Entwicklung der NiO-Anteile und der Texturen in dünnen Schichten zu Beginn der Oxidation beobachtet. 


\subsubsection{Texturentwicklung in dünnen NiO-Schichten}

In der Hochvakuumanlage werden einkristalline Ni-Filme nur wenige Minuten lang oxidiert. Die Filme wurden zunächst im Vakuum auf die Oxidationstemperatur aufgeheizt. Dann wurde Sauerstoff bis zum Erreichen des gewählten Enddrucks in die Kammer eingelassen. Nach Ablauf der Oxidationszeit wurde die Kammer wieder evakuiert und die Substratheizung abgeschaltet. Die Oxidationsparameter wurden so gewählt, dass die Bildung eines sehr hohen NiO(111)-Anteils erwartet wird. Dieser hohe NiO(111)-Anteil wurde nach Oxidationszeiten von 1,12 und 20 Minuten auch beobachtet. Dagegen wurde nach Oxidationszeiten von 3 und 5 Minuten ein sehr hoher NiO(100)-Anteil gemessen. In Abbildung 5.9 ist der $\mathrm{NiO}(100)$-Anteil gegen die Oxidationszeit aufgetragen. Der $\mathrm{NiO}(111)$ ergibt sich wieder aus der Differenz zwischen $100 \%$ und dem NiO(100)-Anteil.

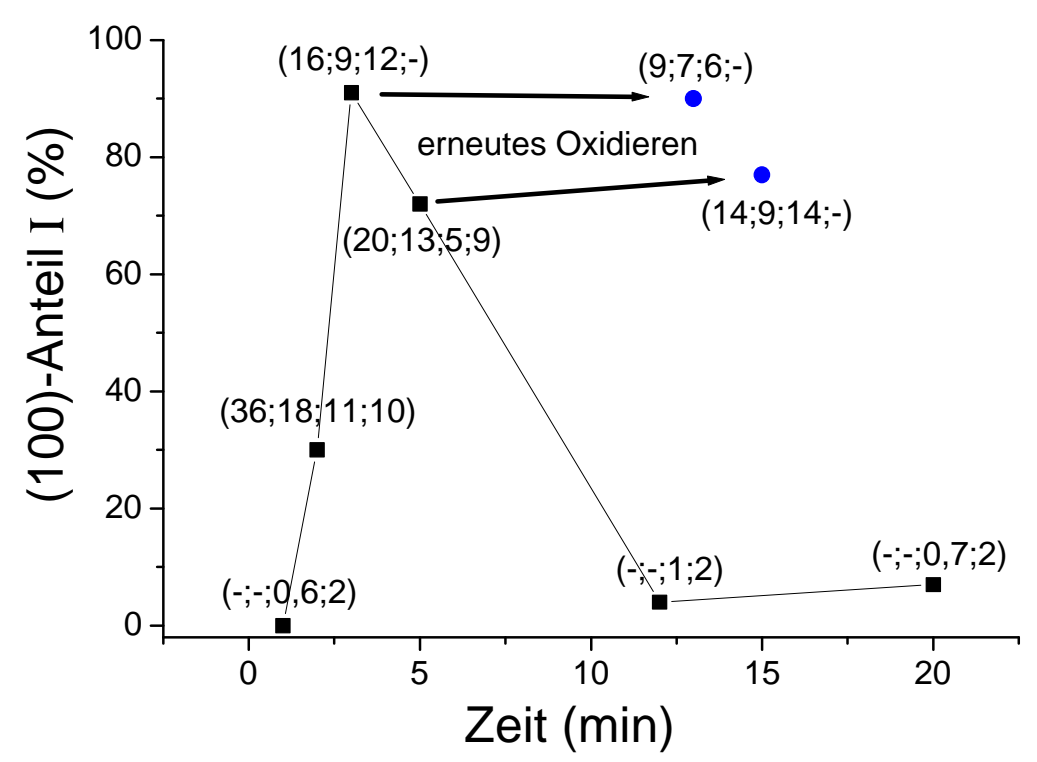

Abb. 5.9 NiO(100)-Anteil in Abhängigkeit von der Oxidationszeit, die Oxidationstemperatur betrug $7^{\circ}{ }^{\circ} \mathrm{C}$ und der Sauerstoffpartialdruck 30 mbar $\mathrm{O}_{2}$. In Klammern sind die Halbwertsbreiten der outof-plane und in-plane Texturen der beiden NiO-Anteile zu jedem Messpunkt geschrieben und zwar in der folgenden Reihenfolge (FWHM(100) out-of-plane ; FWHM(100) in-plane ; FWHM(111) out-ofplane ; FWHM(111) in-plane). Die Pfeile zeigen eine erneute Oxidation der Schichten bei gleichen Parametern an.

Die zum $\mathrm{NiO}(100)$ und $\mathrm{NiO}(111)$-Anteil gehörenden out-of-plane und in-plane Texturen sind als Halbwertsbreiten in Klammern neben den zugehörigen Messpunkt geschrieben. Auffällig ist, dass in einem schmalen Zeitfenster der Oxidation ( $1 \mathrm{~min}<\mathrm{t}<12 \mathrm{~min}$ ) die Schichten hohe (100)-Anteile aufweisen, während ausserhalb dieses Zeitfensters die für dickere Schichten erwartete $\mathrm{NiO}(111)$-Textur auftritt. Die hohen NiO(100)-Anteile bleiben auch bei einer erneuten Oxidation bei gleichen Parametern erhalten. Man muss daher da- 
von ausgehen, dass die Abb 5.9 nicht den zeitlichen Verlauf der Texturbildung anzeigt, sondern dass vielmehr die Abkühlphase nach dem Oxidationsschritt einen signifikanten Einfluss hat. Daraus kann geschlossen werden, dass die NiO-Schichten bei den hier gewählten Oxidationsparametern fast ausschließlich als $\mathrm{NiO}(111)$ wachsen und der $\mathrm{NiO}(100)$-Anteil sich erst während der Abkühlphase, während der sich die Schicht im Vakuum befindet, bildet. In NiO-Schichten mit bestimmter Dicke, findet also im Vakuum eine Umwandlungsreaktion von $\mathrm{NiO}(111)$ in $\mathrm{NiO}(100)$ statt. Die treibende Kraft für diese Umwandlung durchläuft mit zunehmender Oxiddicke anscheinend ein Maximum, da die $\mathrm{NiO}(111)$-Orientierung in dünnen und dicken Schichten stabil ist. Auffällig ist auch, dass die Texturen des NiO(111)-Anteils der Schichten, die fast ausschließlich aus $\mathrm{NiO}(111)$ bestehen, sehr gut sind, nämlich immer besser als $2^{\circ} \mathrm{FWHM}$ sowohl out-of-plane als auch in-plane, während die Texturen aller anderen Schichten sehr viel schlechter sind. Auch dies spricht für eine Nukleation und ein Wachstum von $\mathrm{NiO}(111)$ während der Oxidation und eine spätere Umwandlung in $\mathrm{NiO}(100)$ in der Abkühlphase.

Um die eben entwickelte Vorstellung einer Umwandlungsreaktion von $\mathrm{NiO}(111)$ in $\mathrm{NiO}(100)$ während Abkühlphase zu beweisen, wird dieses Experiment im 2-KreisDiffraktometer wiederholt. Allerdings konnten dort nicht diesselben Parameter eingestellt werden, da der Heizer im 2-Kreis-Diffraktometer bei einem Sauerstoffpartialdruck von 30 mbar lediglich eine Temperatur von $600^{\circ} \mathrm{C}$ erreicht. Bei den eben genannten Parametern wurde der einkristalline Nickelfilm 200 Sekunden lang oxidiert und dann im Vakuum abgekühlt. In Abbildung 5.10 ist diese Messung dargestellt.

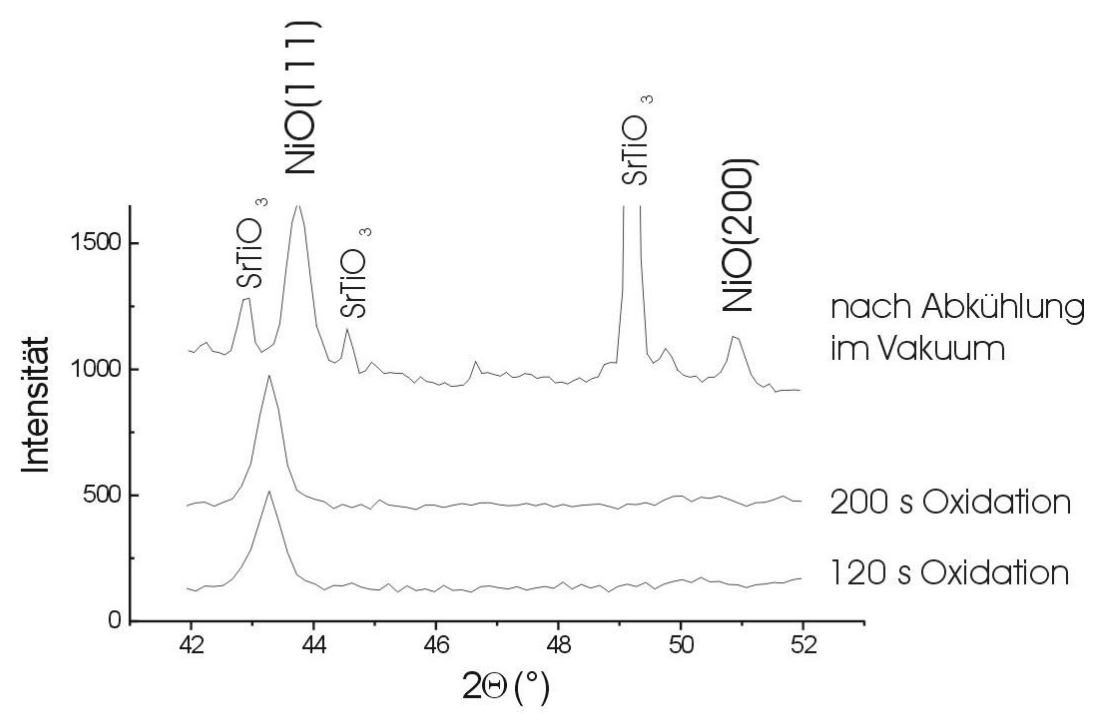

Abb. 5.10 $\Theta-2 \Theta$ Diffraktogramme während und nach 200 Sekunden langer Oxidation. Die Oxidationstemperatur betrug $600^{\circ} \mathrm{C}$, der Sauerstoffpartialdruck 30 mbar. 
Sie bestätigt, dass während der eigentlichen Oxidation ausschließlich $\mathrm{NiO}(111)$ wächst und der $\mathrm{NiO}(100)$-Anteil sich erst während der Abkühlphase gebildet hat. Der Anteil an $\mathrm{NiO}(100)$, der während der Abkühlpase entsteht, beträgt hier 24\% und ist damit nicht so hoch wie in den vorigen Messungen. Dies ist aber dadurch zu erklären, dass andere Parameter verwendet wurden. Dennoch zeigt diese Messung ganz deutlich, dass eine Umwandlungsreaktion von $\mathrm{NiO}(111)$ in $\mathrm{NiO}(100)$ während der Abkühlphase stattfand. Die zeitliche Entwicklung dieser Umwandlung zeigen die Abbildungen 5.11a,b. Hier wird zunächst eine sehr dünne NiO-Schicht mit relativ hohem $\mathrm{NiO}(111)$-Anteil hergestellt. Dies wird erreicht, indem der einkristalline Film 80 Sekunden lang bei $580^{\circ} \mathrm{C}$ und $5 \cdot 10^{-1} \mathrm{mbar}$ $\mathrm{O}_{2}$ oxidiert wird. Danach wird der Film weiter im Vakuum $\left(1 \cdot 10^{-5}\right.$ mbar $) 90$ Minuten lang ausgelagert. In der Abbildung 5.11a ist die zeitliche Entwicklung des $\mathrm{NiO}(100)$-Anteils aufgetragen. Er steigt während der Auslagerung von ca. $35 \%$ auf über $60 \%$ an. In der Abbildung 5.11b sind die Intensitäten der einzelnen Reflexe und die Gesamtintensität der Reflexe gegen die Zeit aufgetragen.

a)

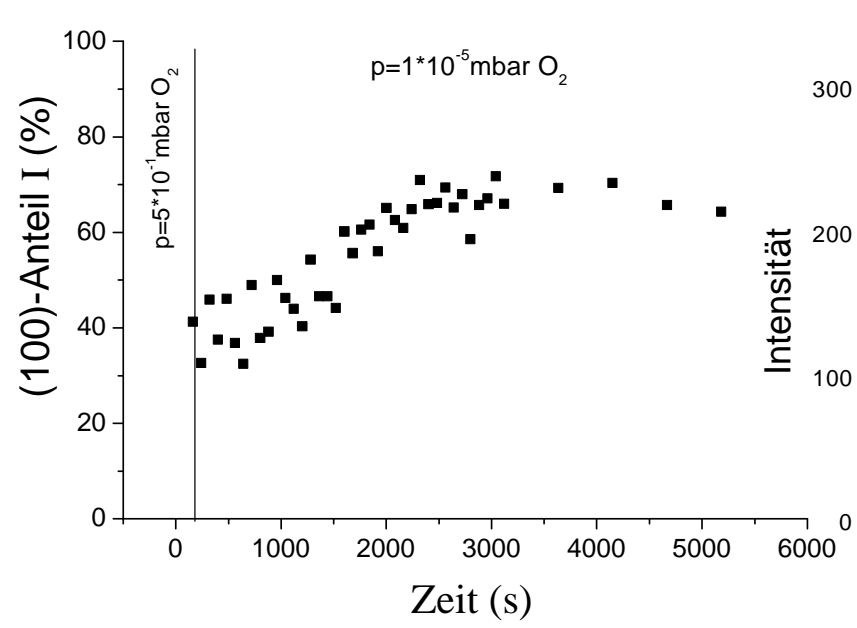

b)

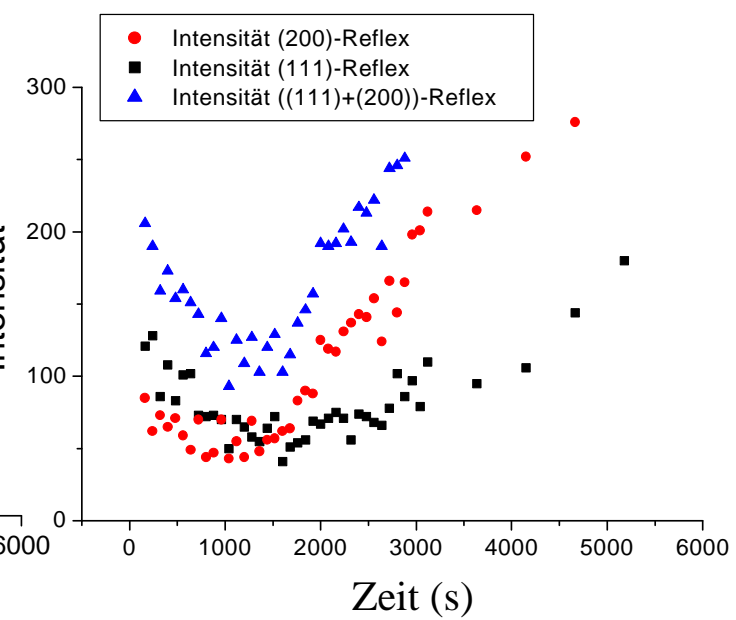

Abb. 5.11 a) $\mathrm{NiO(100)}$ Anteil in Abhängigkeit von der Zeit bei einer Auslagerungstemperatur von $580^{\circ} \mathrm{C}$. b) Intensitäten der einzelnen Reflexe und Gesamtintensität derselben Messung.

Nach langen Zeiten (3000 s) ist zu erkennen, das die Gesamtintensität beider Reflexe stark anwächst. Das Vakuum war also nicht ausreichend, um eine weitere Oxidation zu verhindern. Direkt nach der Druckabsenkung auf $1 \cdot 10^{-5}$ mbar fällt die Intensität des $\mathrm{NiO}(111)$ Reflexes stark ab, bis nach ca 1500 s die Intensitäten des $\mathrm{NiO}(111)$ und des $\mathrm{NiO}(200)$ Reflexes ungefähr gleich groß sind, danach steigt die Intensität des (200)-Reflexes sehr viel stärker an als die des $\mathrm{NiO}(111)$-Reflexes. Die Gesamtintensität der Reflexe nimmt während der Umwandlungsreaktion stark ab. Dies kann mehrere Gründe haben. Es könnte zum Beispiel ein Teil der Schicht wieder abdampfen, oder die Schicht war zunächst relativ glatt und wird während der Umwandlungsreaktion sehr viel rauer. Eine sehr viel ein- 
leuchtendere Erklärung wäre aber, dass der $\mathrm{NiO}(111)$-Anteil zunächst sehr gut texturiert ist und während der Umwandlung eine Texturverschlechterung stattfindet. Diese Interpretation wird auch durch die Abb. 5.9 gestützt, die zeigt, dass in Schichten mit hohem $\mathrm{NiO}(111)$-Anteil die (111) Textur deutlich ausgeprägter ist als die (100)-Textur von Schichten mit hohem (100)-Anteil. Die verschiedenen Messungen zusammen betrachtet, lassen also zum einen den Schluss zu, dass die Umwandlungsreaktion mit einer Texturverschlechterung verbunden ist, und zum anderen, dass aufgrund der sehr guten Textur die Nukleation aus reinem epitaktischem $\mathrm{NiO}(111)$ besteht.

\subsection{Oberflächentopologie und Rauigkeit von NiO-Filmen}

\subsubsection{Die Frühstadien der Rauigkeitsentwicklung}

Die verwendeten Messmethoden erlauben keinen Rückschluss auf den Mechanismus der Rekristallisation in dünnen NiO-Lagen. Einen Hinweis gibt aber das folgende Experiment. Es wurde eine $10 \mathrm{~nm}$ dicke NiO-Schicht mittels reaktiven Ionenstrahlsputterns bei Raumtemperatur auf einen einkristallinen Nickelfim abgeschieden. Diese Schicht war so dünn, dass die Textur mittels Röntgendiffraktometrie nicht bestimmt werden konnte. S. Dreyer [42] beobachtete jedoch an $\mathrm{Ni} / \mathrm{NiO} 70 \cdot(8 \mathrm{~nm} / 8 \mathrm{~nm})$ Multilagen ein epitaktisches Wachstum von $\mathrm{Ni}(100) / \mathrm{NiO}(100)$, das sich säulenartig in alle Lagen fortsetzt. Die einzelnen Säulen jedoch sind innerhalb der Ebene gegeneinander verdreht, so dass makroskopisch lediglich eine (100)-Drahttextur beobachtet wird. Da die Multilagen in derselben Ionenstrahlsputteranlage und unter denselben Depositionsbedingungen hergestellt wurden und

a)

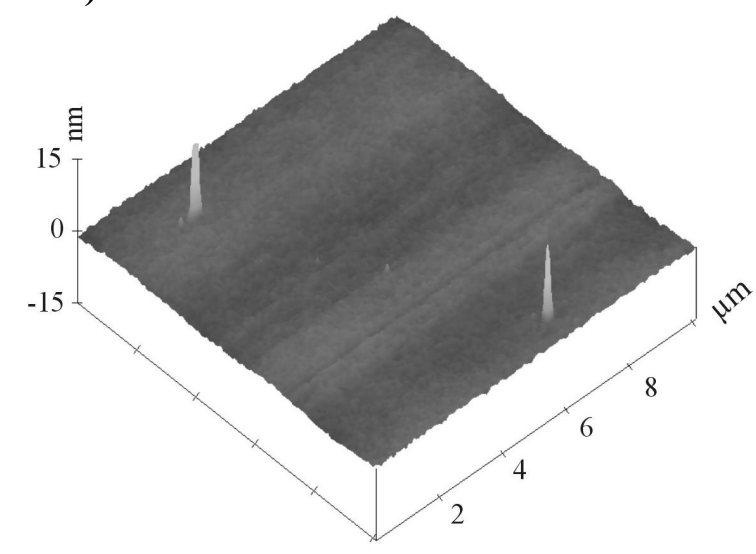

b)

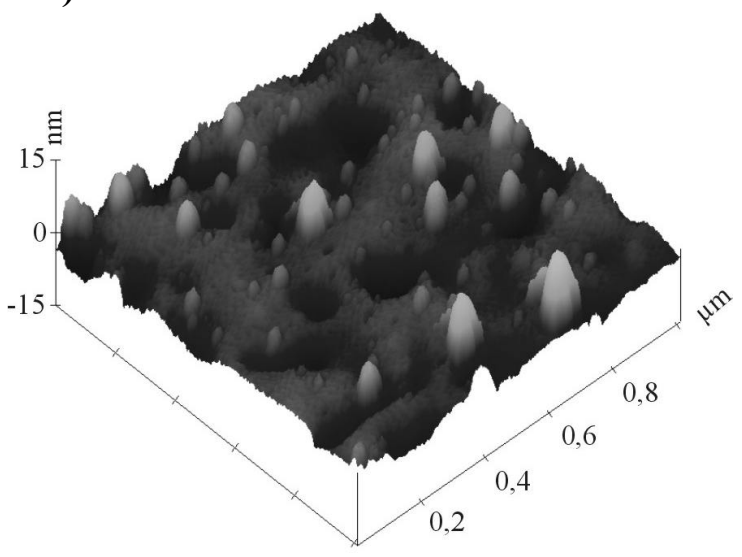

Abb. 5.12 a) AFM-Bild der 10-nm dicken gesputterten NiO-Schicht auf Ni.

b) AFM-Bild der in

a) dargestellten Schicht nach einstündiger Auslagerung im Vakuum bei $500^{\circ} \mathrm{C}$. 
da die Dicke der einzelnen NiO-Schichten fast identisch mit der hier hergestellten Schicht ist, ist davon auszugehen, dass die hier betrachtete Schicht ebenfalls eine NiO(100)Drahttextur aufweist. Die abgeschiedene Schicht wurde mittels AFM untersucht und ist in Abbildung 5.12a dargestellt. Diese Schicht ist sehr glatt, der Mittenrauwert $R_{a}$ beträgt 0,4 $\mathrm{nm}$. Diese NiO-Schicht wurde bei $500^{\circ} \mathrm{C}$ eine Stunde lang im Vakuum, das besser als $1 \cdot 10^{-6}$ mbar war ausgelagert. Die Abbildungen $5.12 \mathrm{~b}$ und 5.13 zeigen die ausgelagerte NiO-Schicht. Sie weist Berge und Löcher auf, die im Mittel immer gleich hoch bzw. tief sind und eine gemittelte Rautiefe von ungefähr $8 \mathrm{~nm}$ aufweisen. Der Mittenrauwert $\mathrm{R}_{\mathrm{a}}$ dieser Schicht beträgt 1,3 $\mathrm{nm}$.
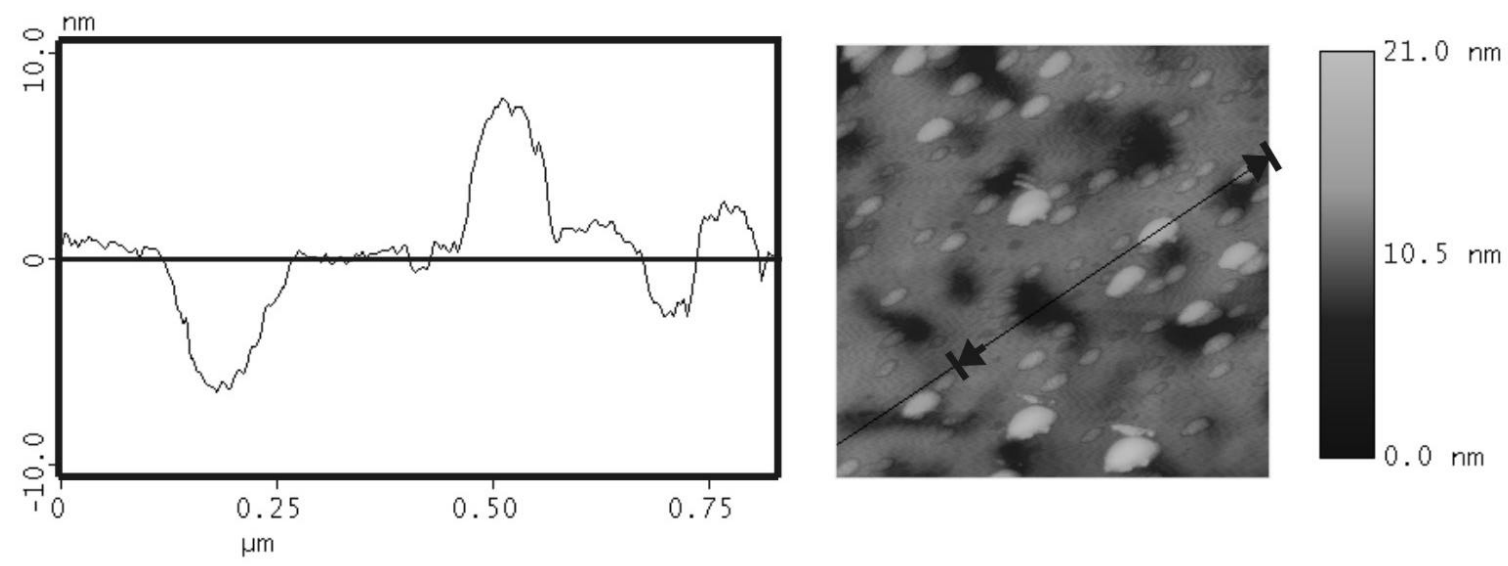

Abb 5.13 AFM-Bilder der $10 \mathrm{~nm}$ dicken bei $500^{\circ} \mathrm{C}$ ausgelagerten NiO-Schicht. Das in der linken $\mathrm{Ab}$ bildung dargestellte Profil ist in der rechten Abbildung mit den schwarzen Pfeilen gekennzeichnet.

Diese Desintegration der dünnen NiO-Lage erinnert an die thermische Zersetzung von Lagenstrukturen in Partikel, wie sie z.B. in Ni/NiO-Schichtpaketen beobachtet wurde [42]. Die treibenden Kräfte für solche Prozesse können lokale Variationen der mechanischen Spannung oder der Grenzflächenenergien sein [43].

\subsubsection{Oberflächentopologie dicker NiO-Schichten}

Obgleich das zum Abschluss des vorigen Kapitels geschilderte Experiment der Desintegration von NiO-Lagen im Vakuum sich nicht direkt auf eine fortschreitende Oxidation übertragen lässt, könnte ein solcher Prozess die Ausbildung der ausgeprägten Rauigkeit der NiO-Schichten initiieren. Eine für dickere Oxidschichten typische Oberflächenstruktur ist in Abbildung 5.14 dargestellt. Die Oberflächen weisen eine ,mäanderartige“ Struktur auf und sind sehr rau. 


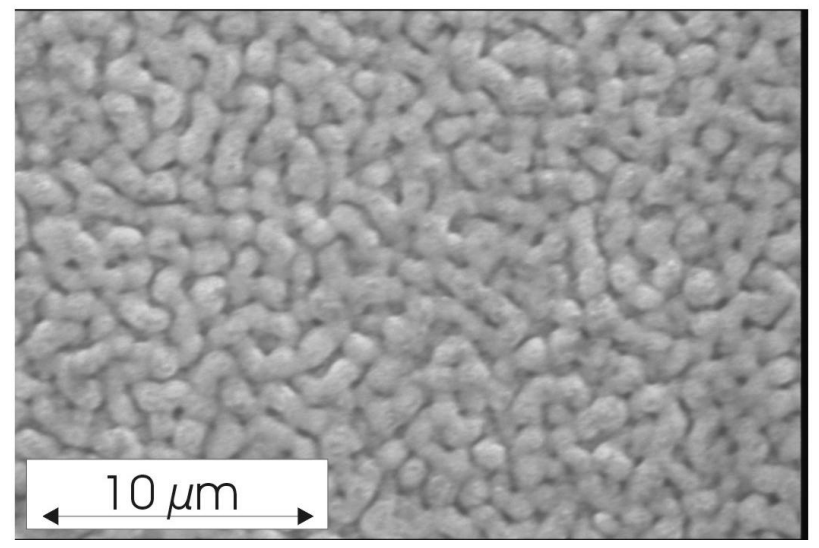

Abb. 5.14 REM-Aufnahme der Oberfläche eines im 2-Kreisdiffraktometer oxidierten einkristallinen Nickelfilms. Die Oxidationstemperatur betrug $600^{\circ} \mathrm{C}$, der Sauerstoffpartialdruck betrug $2 \cdot 10^{-3} \mathrm{mbar}^{-}$ $\mathrm{O}_{2}$, die Oxidationszeit betrug 3 Stunden.

Diese Rauigkeit wurde mittels AFM genauer beobachtet und ist in den Abbildungen 5.15 und 5.16 dargestellt.

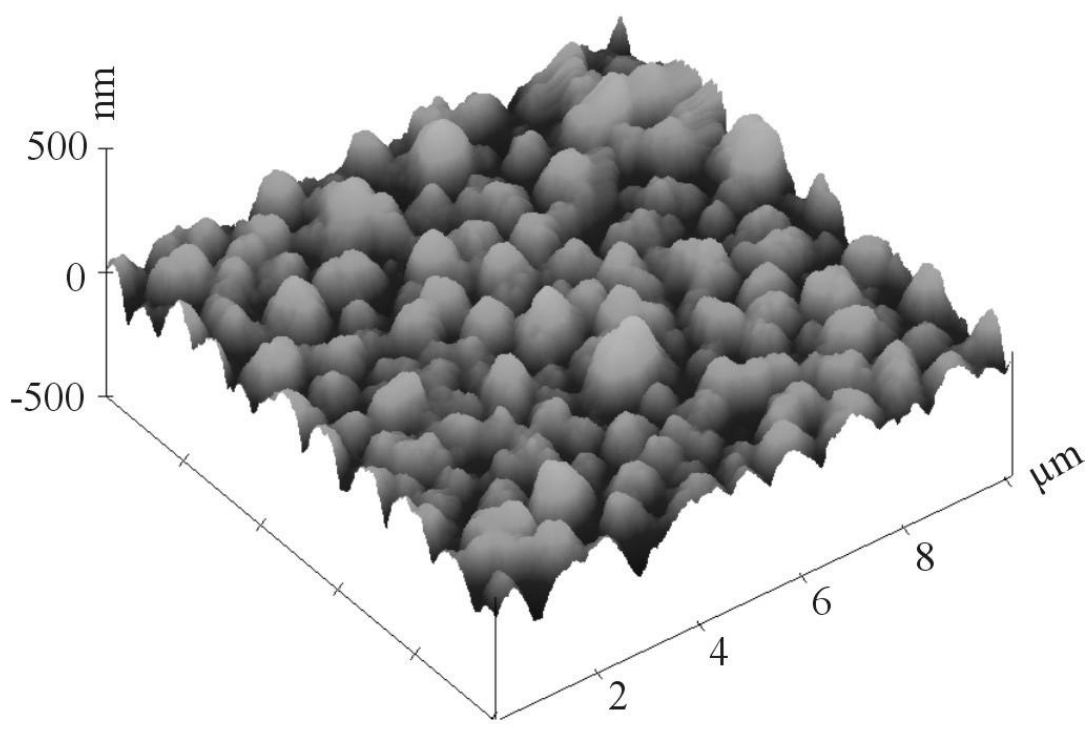

Abb. 5.15 AFM-Bild eines 3 Stunden lang unter $2 \cdot 10^{-3}$ mbar $\mathrm{O}_{2}$ bei $580^{\circ} \mathrm{C}$ oxidierten Nickelfilms.

Der Mittenrauwert $\mathrm{R}_{\mathrm{a}}$ für diesen Film beträgt $45 \mathrm{~nm}$ und der Mittenrauwert $\mathrm{R}_{\mathrm{q}}\left(\mathrm{R}_{\mathrm{MS}}\right)$ beträgt $55 \mathrm{~nm}$. Die Einzelrautiefen liegen aber wie die Abbildung 5.16 zeigt zwischen 280 und $350 \mathrm{~nm}$, so dass die gemittelte Rautiefe $\mathrm{R}_{\mathrm{z}}$ die Oberflächen dieser Filme besser beschreibt. Die Abbildung 5.16 zeigt zusätzlich, dass die Rauigkeitsmaxima nicht wirklich rund sind, sondern ansatzweise eine quadratische Struktur aufweisen, welche durch das epitaktische Wachsen des Oxids auf dem Film erklärt werden kann. 


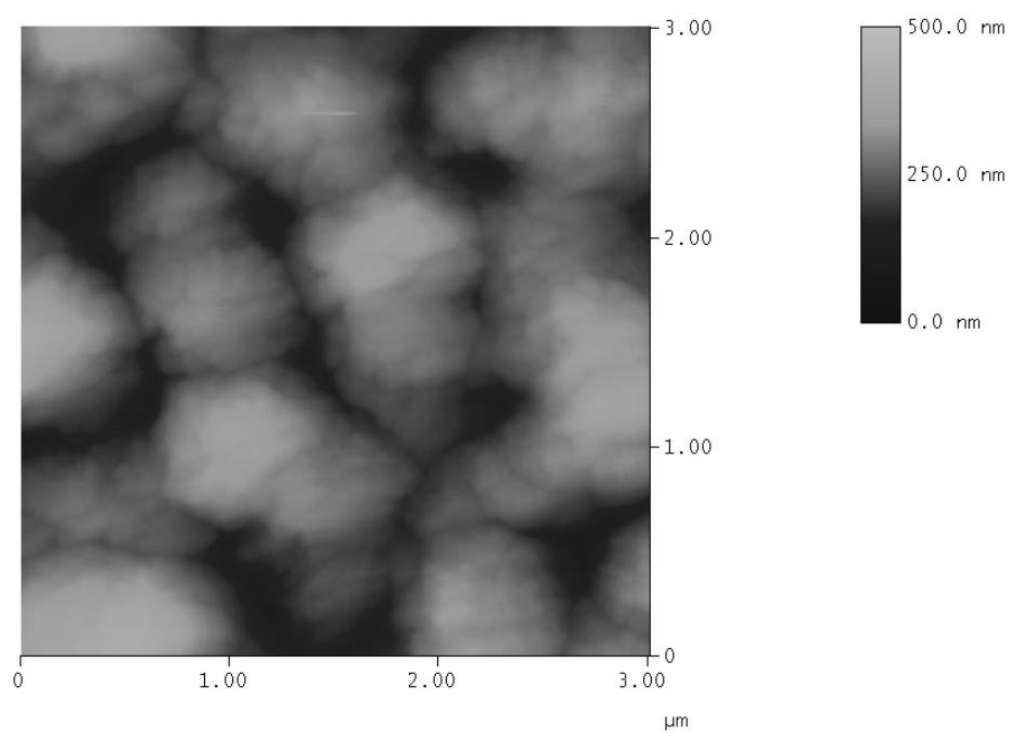

Abb. 5.16 AFM-Bild eines 3 Stunden lang unter $2 \cdot 10^{-3}$ mbar $\mathrm{O}_{2}$ bei $580^{\circ} \mathrm{C}$ oxidierten Nickelfilms.

In den folgenden Experimenten soll nun die Rauigkeitsentwicklung im Verlauf der Oxidation näher untersucht werden. Eine direkte Messung der Oxidschichtdicke war allerdings nicht möglich, da die Schichten zu rau und/oder zu dick sowohl für die Röntgenkleinwinkelstreuung als auch für die Schichtdickenbestimmung mittels Rutherford-BackScattering (RBS) waren. Deshalb wurden die gemittelten Rautiefen $\mathrm{R}_{\mathrm{z}}$ zum einen in $\mathrm{Ab}$ hängigkeit von der Zeit Abb. 5.17a und zum anderen in Abhängigkeit von der Oxidationstemperatur Abb. 5.17b bestimmt. Da die Oxidationstemperatur einen erheblichen Einfluss auf die Schichtdicke hat, zeigt die in Abb 5.17b dargestellte Messung nicht nur die Abhängigkeit von der Oxidationstemperatur, sondern auch die Abhängigkeit von der Oxidschichtdicke. Beide eben genannten Parameter sowohl die Oxidationszeit als auch die Oxidationstemperatur lassen sich unter bestimmten Bedingungen in Schichtdickenverhältnisse umrechnen. Diese Berechnung und die Abhängikeit der gemittelten Rautiefe von der Schichtdicke werden in der Diskussion 6.4 ausführlich besprochen. Die mit dem Profilometer gemessene gemittelte Rautiefe $\mathrm{R}_{\mathrm{z}}$ liegt bei $580^{\circ} \mathrm{C}$ bei der im 2-Kreis-Diffraktometer oxidierten Probe bei ungefähr $300 \mathrm{~nm}$, dieser Wert stimmt gut mit dem aus dem AFM-Bild abgeschätzen $\mathrm{R}_{\mathrm{z}}$ Wert überein. (Die mit dem AFM vermessene NiO-Schicht wurde unter denselben Oxidationsbedingungen hergestellt, wie die eben angesprochene Probe). Die Mittenrauwerte $\mathrm{R}_{\mathrm{a}}$ der in Abbildung 5.17b gemessenen Filme liegen zwischen $12 \mathrm{~nm}$ und $50 \mathrm{~nm}$, auch diese Werte passen gut zu dem im AFM beobachteten Wert von $45 \mathrm{~nm}$. Die drei in Abb. 5.17 dargestellten Messkurven der ermittelten Rautiefen sind nicht konsistent. Bei fast identischen Oxidationsparametern beträgt die Oxidrauigkeit in Abbildung 5.17a $\mathrm{R}_{\mathrm{z}}=40 \mathrm{~nm}$ und in Abbildung $5.17 \mathrm{~b} \mathrm{R}_{\mathrm{z}}=300 \mathrm{~nm}$. Ursache ist hier, dass Schichten, die bei gleichen Parametern oxidiert wurden, deutlich unterschiedliche Oxiddicken aufweisen können. Die deutlichen Unterschiede zwischen 5.17a und 5.17b sind z.B. auf den Wechsel des verwendeteten Ni-Targets zurückzuführen. Die starke Abhängigkeit der Oxidationsrate 
von Verunreinigungen und die daraus resultierenden Probleme in Bezug auf die Reproduzierbarkeit werden in der Diskussion 6.3.5 nochmals aufgegriffen.

a)

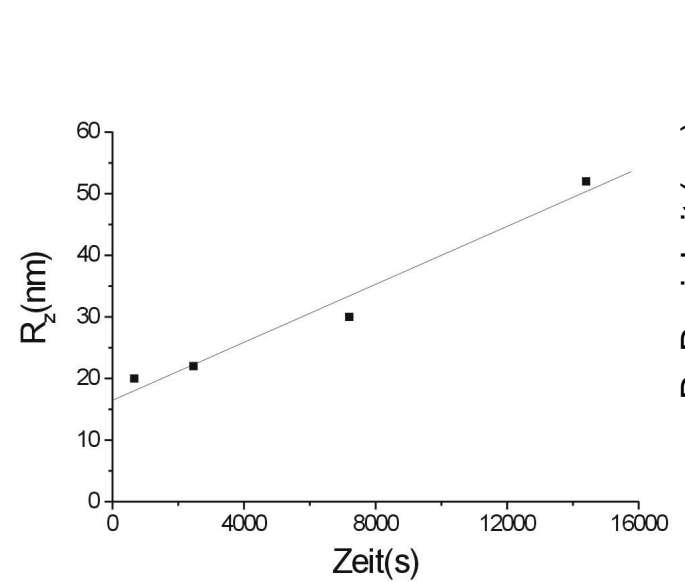

b)

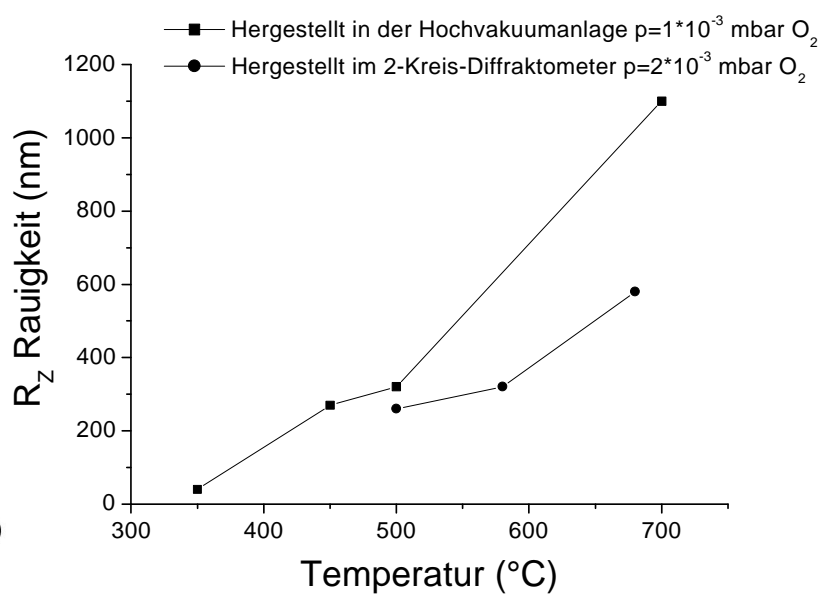

Abb. 5.17 a) Gemittelte Rautiefe $R_{z}$ in Abhängigkeit von der Oxidationszeit, die Oxidationstemperatur betrug $450^{\circ} \mathrm{C}$ und der Sauerstoffpartialdruck $1 \cdot 10^{-3}$ mbar $O_{2}$. b) Gemittelte Rautiefen $R_{z}$ aufgetragen gegen die Oxidationstemperatur. Diese bestimmt erheblich die Schichtdicke des Oxids, die Auftragung zeigt also nicht wirklich eine Abhängigkeit der Rauigkeit von der Temperatur, sondern im wesentlichen eine Abhängigkeit der Rauigkeit von der Schichtdicke. Die Oxidationszeit betrug 3 Stunden.

\subsection{Oberflächen und Schichtaufbau der oxidierten einkristalli- nen NiCr-Filme}

In Abbildung 5.18a,b ist die Oberfläche eines oxidierten NiCr-Films und das oxidierte Schichtsystem im Querschnitt dargestellt.

a)

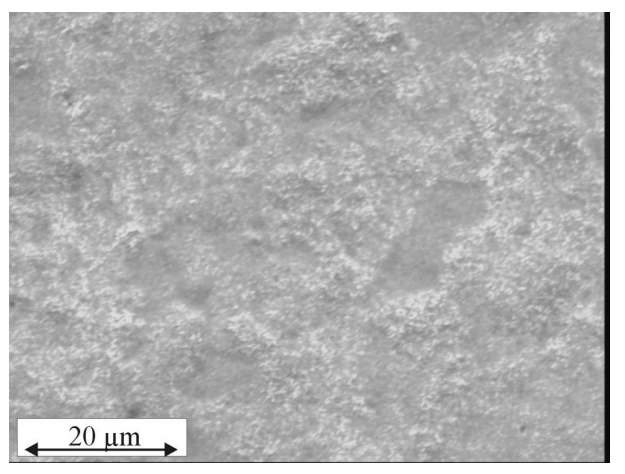

b)

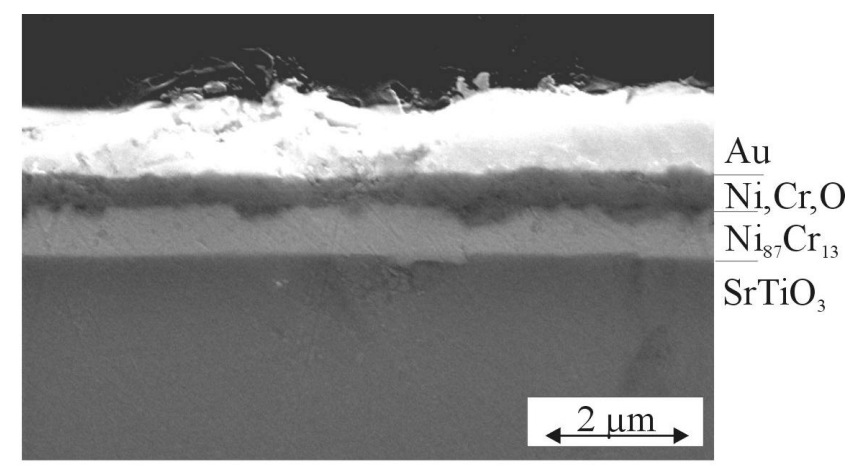

Abb. 5.18 a) REM-Aufnahme der Oberfläche und b) des Querschnitts eines bei $680^{\circ} \mathrm{C}$ und 250 mbar $\mathrm{O}_{2}$ oxidierten NiCr-Filmes, die Oxidationszeit betrug 30 Minuten. 
Die Oberfläche des oxidierten NiCr-Films zeigt eine starke Rauigkeit mit Einzelrautiefen bis zu $220 \mathrm{~nm}$. In der Querschnittsaufnahme ist zu erkennen, dass die innere Grenzfläche zwischen NiCr-Legierung und Oxidschicht eine noch viel höhere Rauigkeit als die äußere Grenzfläche aufweist. Aus dieser Aufnahme wurde die maximale Einzelrautiefe von 320 nm bestimmt. Aufgrund der hohen Rauigkeiten an beiden Grenzflächen ist auch die Schichtdicke der oxidierten Schicht an verschiedenen Stellen unterschiedlich dick, sie beträgt $600 \pm 200 \mathrm{~nm}$. Die oxidierte Schicht sieht homogen aus, kleine fein verteilte Chromoxidausscheidungen oder eine dünne Chromoxidschicht an der inneren Grenzfläche würden aber auch auf dieser Längenskala nicht erkennbar sein.

\subsection{Löcherartige Strukturen in oxidierten einkristallinen Nik- kelfilmen}

Die Oberfläche der oxidierten Nickelfilme weist zumeist die in Abbildung 5.14 dargestellte „mäanderartige“ Struktur auf. Zum Teil werden aber auch ausgesprochen regelmäßige Strukturen beobachtet.

a)

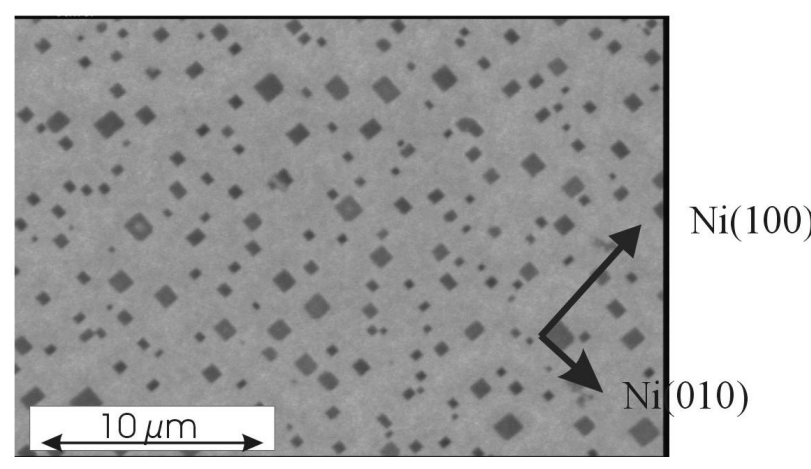

b)

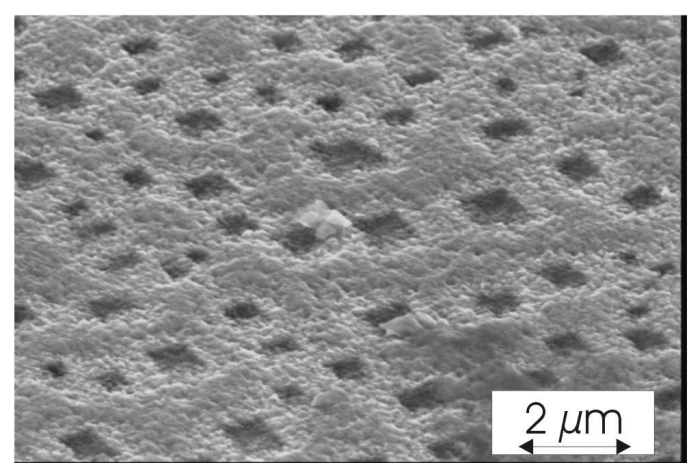

Abb. 5.19 a) REM-Aufnahme eines drei Stunden lang bei $400^{\circ} \mathrm{C}$ und $1 \cdot 10^{-3} \mathrm{mbar} \mathrm{O}_{2}$ oxidierten einkristallinen Nickelfilms b) derselbe Film, betrachtet unter einem schrägen Winkel und bei stärkerer Vergrößerung.

Diese oxidierten Filme zeigen unter bestimmten Oxidationsbedingungen quadratische Vertiefungen. Diese quadratischen Strukturen sind alle entlang der (100) und (010)Richtung des einkristallinen Nickelfilms ausgerichtet. Die Oxidschicht ist aber auch zwischen den Vertiefungen sehr rau. Die Abbildung 5.20 zeigt eine Querschnitts-TEMAufnahme, die diese quadratischen Vertiefungen aufweist. Die Querschnittsaufnahmen zeigen, dass der Nickelfilm nach der Oxidation nicht mehr überall einkristallin ist, sondern dass sich quaderförmige Bereiche gebildet haben, die ein amorphes Beugungsbild aufweisen. Über diesen Bereichen ist die darüberliegende NiO-Schicht sehr viel dünner als die NiO-Schicht über den einkristallinen Bereichen. Wie die REM-Aufnahmen zeigen, sind 
diese Vertiefungen ebenfalls quadratisch. Die darüberliegende Goldschicht wurde als Markerschicht für die Oberfläche des NiO abgeschieden. Das Beugungsbilder der NiOSchicht zeigen sowohl $\mathrm{NiO}(111)$ als auch $\mathrm{NiO}(200)$-Reflexe und sind im wesentlichen unabhängig davon, an welcher Stelle der NiO-Schicht sie aufgenommen wurden. Die Abbildung 5.21 zeigt die NiO-Schicht im Querschnitt mit größerer Vergrößerung. Deutlich zu erkennen sind hier die einzelnen Körner, die eine Größe von 50-250 nm aufweisen und etliche Korngrenzen.

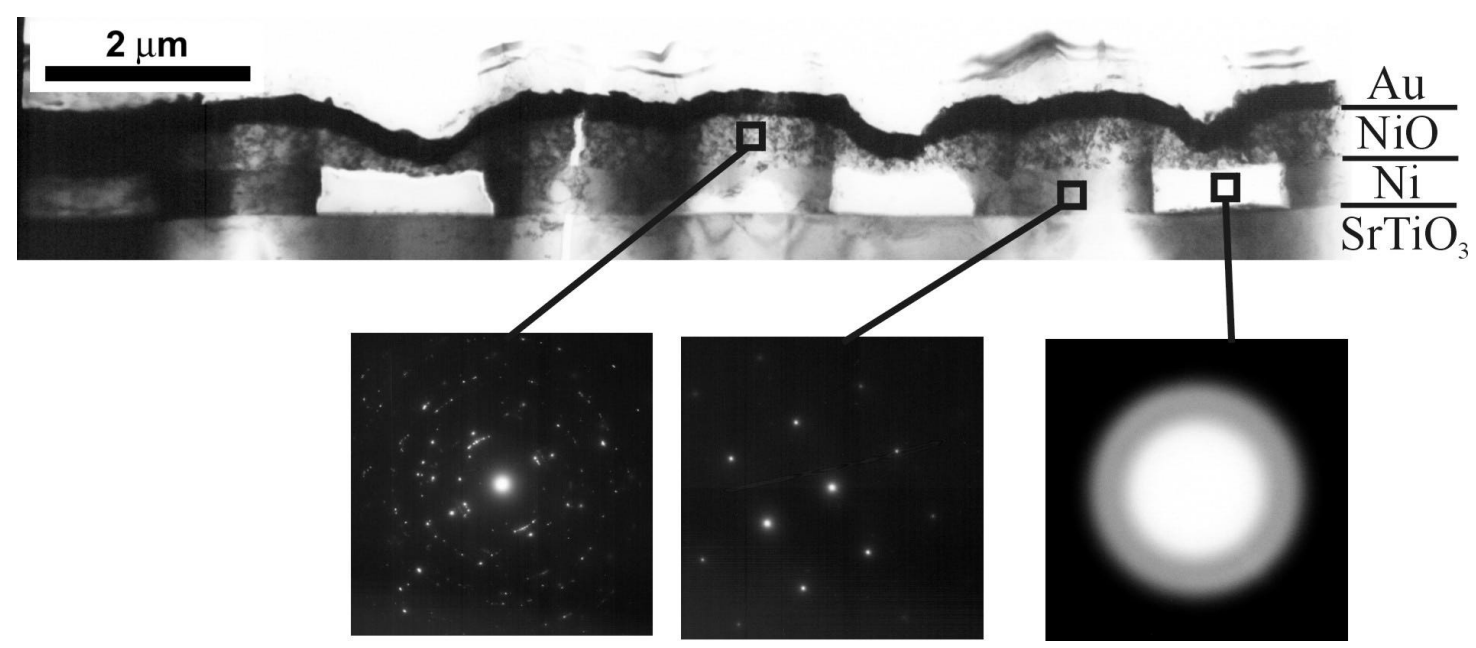

Abb. 5.20 TEM-Querschnitts-Aufnahme eines 6 Stunden lang bei $450^{\circ} \mathrm{C}$ und $1,3 \cdot 10^{-3}$ mbar $\mathrm{O}_{2}$ oxidierten einkristallinen Nickelfilms.

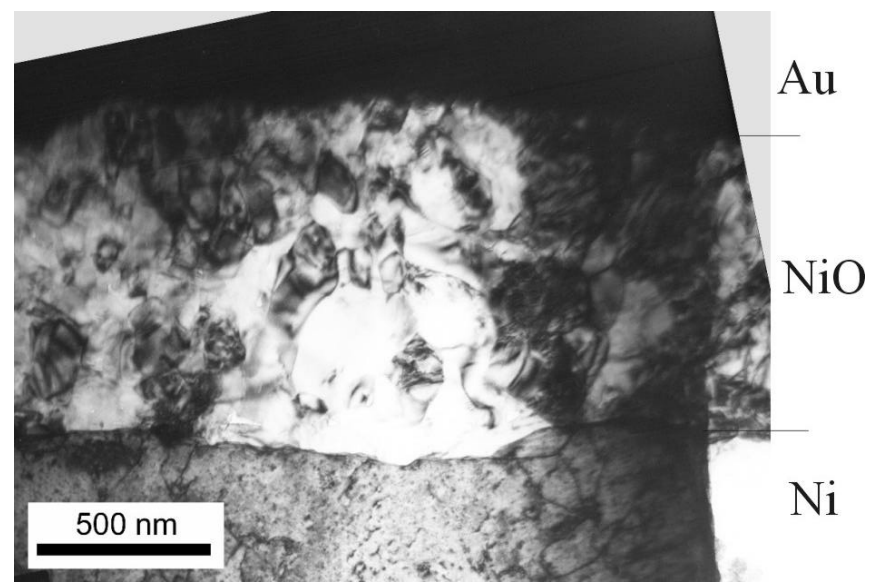

Abb. 5.21 TEM-Querschnitts-Aufnahme der in Abbildung 5.13 dargestellten Probe unter größerer Vergrößerung. 


\subsection{Beschichtung YBaCuO}

Auf den Eigenoxidschichten des Ni und NiCr mit den höchsten $\mathrm{NiO}(100)$-Anteilen wurden mittels PLD bei $740^{\circ} \mathrm{C}$ und 0,6 mbar $\mathrm{O}_{2}$ YBaCuO-Filme abgeschieden. Es wuchs zwar die erwartete $\mathrm{Y}_{1} \mathrm{Ba}_{2} \mathrm{Cu}_{3} \mathrm{O}_{7-\mathrm{x}}$-Phase auf, diese zeigt aber keine Textur. Die Rautiefen der abgeschiedenen YBaCuO-Schicht zeigten ähnliche Werte wie die darunterliegenden Oxidschichten. Die Rauigkeit einkristalliner bei niedrigen Temperaturen oxidierter Filme ist immer noch zu hoch für eine epitaktische Deposition. Auch das epitaktische Abscheiden von weiteren Pufferschichten wie z.B. $\mathrm{MgO}, \mathrm{Pt}, \mathrm{CeO}_{2}, \mathrm{CGO}$ und $\mathrm{YSZ}$ auf den Eigenoxiden ist aufgrund der hohen Rauigkeiten gescheitert und führte nicht zu einer Glättung der Filme.

Schließlich wurde ein glatter gesputterter einkristalliner $\{100\}<100>$ NiO-Film mit $\mathrm{YBaCuO}$ beschichtet. Der gesputterte einkristalline NiO-Film wurde bei $400^{\circ} \mathrm{C}$ epitaktisch auf $\mathrm{SrTiO}_{3}(100) \mathrm{K}$ abgeschieden und weist sowohl out-of-plane als auch in-plane Texturen von unter $1^{\circ} \mathrm{FWHM}$ auf, die mittlere Rautiefe lag weit unter $\mathrm{R}_{\mathrm{z}}<10 \mathrm{~nm}$. Der abgeschiedene YBaCuO-Film wuchs epitaktisch in der Y123-Phase mit Texturen die Halbwertsbreiten von unter $1^{\circ} \mathrm{FWHM}$ aufwiesen auf dem einkristallinem $\mathrm{NiO}$ auf. Eine induktive Messung ergab, dass der Film eine Sprungtemperatur von $84 \mathrm{~K}$ und eine kritische Stromdichte von $\mathrm{j}_{\mathrm{c}}=5 \cdot 10^{4} \mathrm{~A} / \mathrm{cm}^{2}$ besitzt. Dieses Experiment zeigt, dass es generell möglich ist, supraleitende Filme auf $\mathrm{NiO}$ abzuscheiden. Allerdings liegen die hier gemessene Sprungtemperatur und die kritische Stromdichte noch unter den Minimalanforderungen von $\mathrm{j}_{\mathrm{c}}=5 \cdot 10^{5} \mathrm{~A} / \mathrm{cm}^{2}(77 \mathrm{~K}, 0 \mathrm{~T})$ für supraleitende Bänder. Jedoch waren die Beschichtungsparameter auch nicht für das $\mathrm{NiO}$, sondern für den YSZ-Puffer optimiert. 


\section{Diskussion}

\subsection{Oxidation von Metallen}

Die Metalloxidation, die auch als Verzunderung oder Anlaufen bezeichnet wird, kann in ihren Grundzügen in die folgenden Einzelschritte unterteilt werden [35].

1. Sauerstoff chemisorbiert an der äußeren Grenzfläche (Metalloxid/Gasatmosphäre) und wird dabei zu O $\mathrm{O}^{2-}$ Anionen reduziert. $\left(1 / 2 \mathrm{O}_{2}+2 \mathrm{e}^{-} \rightarrow \mathrm{O}^{2-}\right)$

2. An der inneren Grenzfläche (Metall-Metalloxid) geht das Metall unter Abgabe von Elektronen als Kation in die Oxidphase über. $\left(\mathrm{Me} \rightarrow \mathrm{Me}^{2+}+2 \mathrm{e}^{-}\right)$

3. Die chemisorbierten $\mathrm{O}^{2-}$ Anionen diffundieren zur inneren Grenzfläche (MetallMetalloxid) und die Metallkationen und Elektronen zur äußeren Grenzfläche (Metalloxid-Gasphase).

Die Bildung des Metalloxides findet in der Theorie von Carl Wagner [44,35] entweder an der inneren oder an der äußeren Grenzfläche statt.

Ist die Beweglichkeit der Metallkationen in dem Metalloxid sehr viel größer als die Beweglichkeit der Sauerstoffanionen, so findet die Oxidation im wesentlichen an der äußeren Grenzfläche statt. Dazu müssen sowohl die Metallkationen als auch die Elektronen durch das Metalloxid diffundieren. Die chemisorbierten Sauerstoffanionen reagieren dann mit den Metallkationen zum Oxid. Im umgekehrten Fall, also falls die Beweglichkeit der Sauerstoffanionen sehr viel größer ist als die der Metallkationen, bildet sich das Oxid an der inneren Grenzfläche. Hierbei diffundieren die bei der Bildung der Metallkationen entstandenen Elektronen zur äußeren Grenzfläche und die Sauerstoffanionen zur inneren Grenzfläche, wobei das Oxid ladungsneutral bleibt.

Das Wachstum von Oxidschichten setzt treibende Kräfte voraus. Unterschiedliche treibende Kräfte führen zu verschiedenen Wachstumsgesetzen. Ist die treibende Kraft der Konzentrationsgradient der beteiligten Elemente, und ist der Sauerstoffdruck konstant und ist die Reaktionsgeschwindigkeit der Oxidphase schneller als die Diffusionsgeschwindigkeit, so ist das Wachstum diffusionskontrolliert und kann mit Hilfe der Fickschen Gesetze beschrieben werden. Der zeitliche Verlauf des Oxidwachstums folgt in diesem Fall einem parabolischen Wachstumsgesetz $d^{2}=2 k_{p} t$, dabei ist $d$ die Oxidschichtdicke, $k_{p}$ die Zun- 
derkonstante und t die Zeit. Unter den eben genannten Bedingungen sollten die Zunderkonstante und die Diffusionskonstante identisch sein. Der Leerstellenmechanismus ist der vorherrschende Mechanismus der Diffusion [45]. Die freie Bildungs- und Wanderungsenergie von Leerstellen $\left(\mathrm{F}_{\text {Wanderung }}=\mathrm{E}_{\text {Wanderung }}-\mathrm{T} \mathrm{S}_{\text {Wanderung }}\right)$ bestimmen also die Diffusionskonstante. Beide Energien werden thermisch aktiviert, die Diffusionskonstante ist also $\mathrm{D}=\mathrm{D}_{0} \exp \left(-\left(\mathrm{E}_{\text {Bildung }}+\mathrm{E}_{\text {Wanderung }}\right) / \mathrm{kT}\right)$. Die Entropieterme sind weitgehend unabhängig von der Temperatur und sind in der Konstante $\mathrm{D}_{0}$ zusammengefasst. Das parabolische Wachstumsgesetz gilt nicht für sehr dünne Anlaufschichten und niedrige Temperaturen. In diesen Fällen erzeugen elektrische Felder, die durch die adsorbierten Sauerstoffionen und angereicherte geladene Leerstellen hervorgerufenen werden, ebenfalls eine treibende Kraft. Werden solche Raumladungszonen mit berücksichtigt, so erhält man je nach Leitungsmechanismus in der Oxidschicht kubische oder logarithmische Wachstumsgesetze [35].

\subsubsection{Oxidation von $\mathrm{Ni}$}

Da die Beweglichkeit von $\mathrm{Ni}^{2+}$-Ionen im NiO-Gitter wesentlich größer ist als die Beweglichkeit von $\mathrm{O}^{-}$-Ionen, diffundiert hauptsächlich das Nickel an die äußere Grenzfläche, und die Reaktion zu NiO findet hauptsächlich dort statt, die NiO-Schicht wächst also nach außen. Im Nickeloxidgitter befinden sich zweifach negativ geladene Metallionenleerstellen $\mathrm{Ni}$ " und eine äquivalente Anzahl an $\mathrm{Ni}^{3+}$-Ionen, auch Defektelektronen $\oplus$ genannt. Bei der Chemisorption des Sauerstoffs muss sich folglich ein Defektelektron bilden $\left(1 / 2 \mathrm{O}_{2} \rightarrow \mathrm{O}^{-}+\oplus\right)$, dann erfolgt der Anbau von NiO-Molekülen. Dabei ensteht eine Nikkelleerstelle und ein Defektelektron $\left(\mathrm{O}^{-} \rightarrow \mathrm{NiO}+\mathrm{Ni} "+\oplus\right)$. Bei hohen Temperaturen erfolgt unmittelbar nach der Chemisorption der Anbau des Nickeloxids, so dass beide Gleichungen zusammengefasst werden können $\left(1 / 2 \mathrm{O}_{2} \rightarrow \mathrm{NiO}+\mathrm{Ni} " \prime+2 \oplus\right)$. Aus der Massenwirkungsgleichung folgt, dass die Konzentration an Nickelleerstellen proportional zur 6. Wurzel des Sauerstoffpartialdruckes ist. Wird von einer Diffusion über Leerstellen ausgegangen, so sollte auch die Diffusions bzw. Zunderkonstante proportional zur 6. Wurzel des Sauerstoffpartialdruckes sein $\left(k \sim \mathrm{p}^{1 / 6}\right)$. Diese Abhängigkeit wird in der Tat von einigen Gruppen gefunden [46]. Andere Gruppen hingegen finden im selben Temperaturbereich (1000-1400 K) noch stärkere Sauerstoffdruckabhängigkeiten bis hin zu einer Proportionalität der 4. Wurzel zur Zunderkonstanten. Die Wachstumskinetik des Nickeloxids ist also nicht nur von der Temperatur, sondern ebenfalls vom Sauerstoffpartialdruck abhängig. 


\subsubsection{Oxidation von $\mathrm{NiCr}$}

Im Legierungsystem $\mathrm{NiCr}$ ist Nickel die edlere und Chrom die unedlere Legierungskomponente. Je nach Legierungszusammensetzung, Temperatur und Sauerstoffpartialdruck ist die Oxidbildung unterschiedlich. Viele Gruppen wie z.B. Gulbransen et al.[47] und Atsushi Takei und Kazuyoshi Nii [48] haben die Oxidbildung im System $\mathrm{Ni}_{80} \mathrm{Cr}_{20}$ im Temperaturbereich zwischen $500^{\circ} \mathrm{C}$ und $1000^{\circ} \mathrm{C}$ an Luft sehr detailiert untersucht. Obwohl Chrom das unedlere Metall ist, bildet sich in den ersten Minuten der Oxidation aufgrund der höheren Reaktionsgeschwindigkeit NiO. Mit wachsender NiO-Schicht nimmt die Sauerstoffaktivität an der Grenzfläche Legierung-Oxidschicht so stark ab, dass der Dissoziationsgleichgewichtsdruck zur Bildung von $\mathrm{NiO}$ unterschritten ist. Nun findet eine selektive Oxidation des unedleren Chroms an der inneren Grenzfläche statt. Die äußere NiO-Schicht wächst weiter nach außen und wird von einer Chromoxidschicht unterwachsen. Im weiteren Verlauf der Oxidation wird die Chromoxidschicht so dick, dass das Wachstum der äußeren NiO-Schicht zum Erliegen kommt, da die Chromoxidschicht so dicht ist, dass die Diffusion von Nickel durch diese Schicht sehr gering ist. An der Grenzfläche zwischen $\mathrm{NiO}$ und $\mathrm{Cr}_{2} \mathrm{O}_{3}$ findet eine Umwandlungsreaktion $\mathrm{zu} \mathrm{NiCr}_{2} \mathrm{O}_{4}$ statt, so dass folgende Schichtabfolge entsteht (NiO/NiCr$\left.{ }_{2} \mathrm{O}_{4} / \mathrm{Cr}_{2} \mathrm{O}_{3} / \mathrm{Ni}_{80} \mathrm{Cr}_{20}\right)$. Nach sehr langen Oxidationszeiten hatte sich die NiO-Schicht völlig in $\mathrm{NiCr}_{2} \mathrm{O}_{4}$ umgewandelt. Die Bildung von $\mathrm{NiO}$ ist dementsprechend nur bei hinreichend hohen Sauerstoffpartialdrücken möglich. Bei geringeren Chromzusätzen $(\mathrm{Cr}<10 \mathrm{wt} \%)$ finden Moreau und Benard [49,50] nach längerer Oxidationszeiten im Temperaturbereich zwischen $800^{\circ} \mathrm{C}-1300^{\circ} \mathrm{C}$ an Luft eine äußere Zunderschicht die aus NiO-Schicht mit $\mathrm{NiCr}_{2} \mathrm{O}_{4}$-Ausscheidungen besteht, und eine innere Oxidationszone, in der Nickel mit $\mathrm{Cr}_{2} \mathrm{O}_{3}$-Ausscheidungen vorliegt.

Bei kleinen Zusätzen an Chrom $(\mathrm{Cr}<6$ at\%), steigt die Oxidationsgeschwindigkeit erheblich an, da die höherwertigen $\mathrm{Cr}^{3+}$-Kationen in der NiO-Schicht die Leerstellenkonzentration erhöhen und sich somit auch Zunderkonstante erhöht.

Bei höheren Zusätzen dagegen ist die Zundergeschwindigkeit gegenüber der des reinen Nickels sehr stark verringert. Grund dafür sind die entstehenden Nickelchromoxide und Chromoxid, die sich besonders an den Korngrenzen bilden. In diesen Oxiden ist die Beweglichkeit der Metallkationen sehr viel geringer als im $\mathrm{NiO}$.

\subsection{Kinetik der Oxidation von einkristallinen Nickelfilmen}

Die zeitaufgelösten Messungen des $\mathrm{NiO}(100)$-Anteil (Abb 5.5a,b) zeigen, dass bereits nach wenigen Minuten der $\mathrm{NiO}(100)$ und $\mathrm{NiO}(111)$-Anteil über die gesamte restliche Oxidationszeit konstant bleiben. Auch die out-of-plane Textur des $\mathrm{NiO}(100)$-Anteil zeigt über die gesamte Oxidationszeit eine konstante Halbwertsbreite. Selbst starke Veränderun- 
gen der Oxidationsparameter (Temperatur und Sauerstoffpartialdruck) verändern den $\mathrm{NiO}(100)$ und $\mathrm{NiO}(111)$-Anteil nicht mehr (Abb 5.8a, b). Eine sich bereits gebildete NiOSchicht wächst also ab einer gewissen Dicke unabhängig von den Oxidationsparametern epitaktisch weiter. Wie schon bei der Darstellung der Experimente beschrieben, ist bei konstanten $\mathrm{NiO}(100)$-Anteil und konstanter out-of-plane Textur, die Intensität der $\mathrm{NiO}(200)$-Reflexes proportional zur Dicke der NiO-Schicht. Deshalb soll jetzt zunächst die zeitliche Änderung der Oxidschichtdicke beschrieben werden, bevor später auf die Entwicklung der Textur eingegangen wird. Aus dem vorigen Kapitel folgt, dass für das Dikkenwachstum der Oxidschicht im diffusionsbestimmten Fall ein parabolisches Zeitgesetz und eine durch den Boltzmann-Faktor beschriebene Temperaturabhängigkeit erwartet wird. Die Auftragung der Intensität des $\mathrm{NiO}(200)$-Reflexes gegen die Wurzel der Zeit in Abbildung 5.7 lässt sich durch einen linearen Verlauf anfitten und zeigt daher das parabolische Wachstum der NiO-Schicht. Dies bedeutet, dass das Wachstum der Schicht über einen diffusionsgesteuerten Prozess abläuft, bei dem die treibenden Kräfte die Konzentrationsgradienten der Elemente Nickel und Sauerstoff sind. In diesem Fall ist die Oxidationskonstante $\mathrm{k}_{\mathrm{p}}$ gleich der Diffusionskonstanten $\mathrm{D}\left(\mathrm{k}_{\mathrm{p}}=\mathrm{D}\right)$. Wie bereits unter 6.1 beschrieben, ist die Diffusion ein thermisch aktivierter Prozess. Zur Bestimmung der Aktivierungsenergie müssen also die relativen Schichtdicken bei verschiedenen Temperaturen aufgenommen werden. In Abbildung 6.1a ist die Intensität des $\mathrm{NiO}(200)$-Reflexes gegen die Wurzel der Zeit aufgetragen.(vgl Abb. 5.8b). Deutlich zu erkennen ist wieder das parabolische Wachstumsgesetz für die einzelnen Temperaturbereiche und die zunehmenden Steigungen der Geraden mit steigender Temperatur. In Abbildung 6.1b ist der natürliche Logarithmus des Quadrates der unterschiedlichen Geradensteigungen $\ln \left(\mathrm{m}_{1}{ }^{2}\right)$ aus Abb. 6.1a gegen den Kehrwert der Temperatur 1/T aufgetragen. Folgende Gleichungen führen zu der eben beschriebenen Auftragung:

$\mathrm{I}(200) \sim \mathrm{d}=\mathrm{c}_{1}\left(\mathrm{k}_{\mathrm{p}} \mathrm{t}\right)^{1 / 2} \quad$ (parabolisches Wachstumsgesetz)

d: Dicke der NiO-Schicht, I(200): Intensität des (200)-Reflexes

$\mathrm{t}$ : Zeit, $\mathrm{k}_{\mathrm{p}}$ : Oxidationskonstante, $\mathrm{c}_{1}, \mathrm{c}_{2}$ : Konstanten

mit $\mathrm{k}_{\mathrm{p}}=\mathrm{k}_{0} \exp \left(-\mathrm{E}_{\mathrm{A}} / \mathrm{k}_{\mathrm{B}} \mathrm{T}\right)$

wobei $\mathrm{E}_{\mathrm{A}}$ die Aktivierungsenergie ist, folgt mit

$\mathrm{m}_{1}=\mathrm{c}_{1} \mathrm{k}_{\mathrm{p}}^{1 / 2}$

$\ln \left(\mathrm{m}_{1}^{2}\right)=2 \ln \mathrm{c}_{1}+\ln \mathrm{k}_{0}-\mathrm{E}_{\mathrm{A}} /\left(\mathrm{k}_{\mathrm{B}} \mathrm{T}\right)=\mathrm{c}_{2}-\mathrm{m}_{2} / \mathrm{T} \quad$ mit $\quad \mathrm{m}_{2}=\mathrm{E}_{\mathrm{A}} / \mathrm{k}_{\mathrm{B}}$ 
Die Aktivierungsenergie $\mathrm{E}_{\mathrm{A}}$ ergibt sich also aus der Steigung der in Abb 6.1b dargestellten Geraden mit $\mathrm{E}_{\mathrm{A}}=\mathrm{m}_{2} \mathrm{k}_{\mathrm{B}}$ und beträgt $\mathrm{E}_{\mathrm{A}}=1,8 \pm 0,4 \mathrm{eV}$.

a)

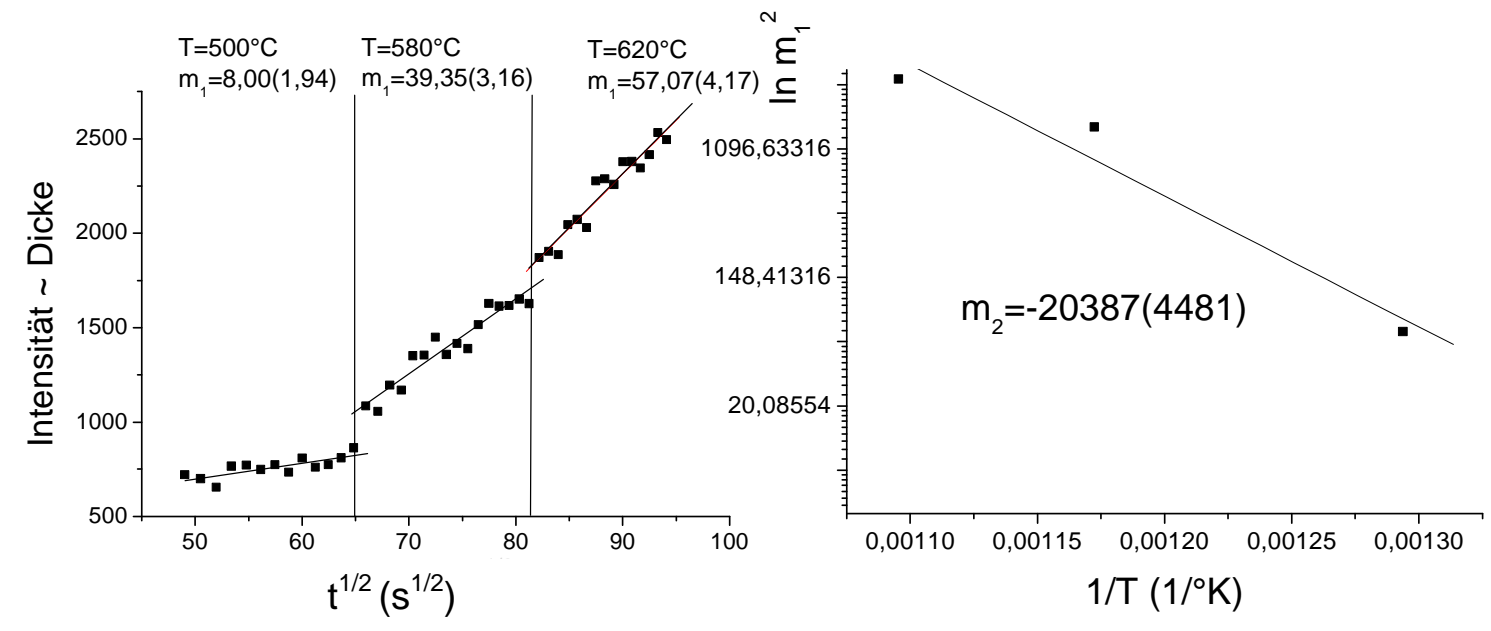

Abb. 6.1 a) Intensität des (200)-Reflexes gegen die Wurzel der Zeit aufgetragen; die Temperatur wurde während der Messung zweimal erhöht.

b) Natürlicher Logarithmus der Quadrate der Geradensteigungen aus a) aufgetragen gegen den Kehrwert der Temperatur. Die aus dieser Steigung ermittelte Aktivierungsenergie beträgt $\mathrm{E}_{\mathrm{A}}=1,8 \pm 0,4 \mathrm{eV}$.

In der Literatur wird bei der Oxidation von Ni(100)-Einkristallen ab einer Temperatur von $400^{\circ} \mathrm{C}$ häufig ebenfalls ein parabolisches Wachstumsgesetz beschrieben. E. Donald und Jr. Larsen [51] z.B. geben bei einer Oxidationstemperatur von $500^{\circ} \mathrm{C}$ für $\mathrm{Ni}(100)$-Einkristalle eine parabolische Zunderkonstante von $\mathrm{k}_{\mathrm{p}}=5,136 \cdot 10^{-15} \mathrm{~cm}^{2} / \mathrm{s}$ an. F. Czerwinski und J.A. Szpunar [52] finden eine parabolische Oxidationskonstante von $\mathrm{k}_{\mathrm{p}}=4,73 \cdot 10^{-15} \mathrm{~cm}^{2} / \mathrm{s}$ bei $450^{\circ} \mathrm{C}$ und $5 \cdot 10^{-3}$ torr $\mathrm{O}_{2}$ bei der Oxidation von $\mathrm{Ni}(100)$-Einkristallen. Allerdings gibt es auch Gruppen, die unterhalb einer Temperatur von $500^{\circ} \mathrm{C}$ kubische Wachstumsgesetze finden [35]. Die in dieser Arbeit bestimmte Aktivierungsenergie von $\mathrm{E}_{\mathrm{A}}=1,8(4) \mathrm{eV}$ passt sehr gut mit einer Reihe von Literaturwerten zusammen. Moore und Lee [53] messen im Temperaturbereich zwischen $400^{\circ} \mathrm{C}$ und $900^{\circ} \mathrm{C}$ eine Aktivierungsenergie von 1,5 eV und A. Atkinson und R.I. Taylor [54] ermitteln eine Aktivierungsenergie von 1,78 eV für den Temperaturbereich von $500^{\circ} \mathrm{C}$ bis $800^{\circ} \mathrm{C}$. Die in der Literatur angegeben Aktivierungsenergien für höhere Oxidationstemperaturen von über $1000^{\circ} \mathrm{C}$ sind höher und liegen zwischen 2,2 eV und 2,6 eV. Beispielsweise geben R. Lindner und Å. Åkerström [55] eine Aktivierungsenergie von $2,4 \mathrm{eV}$ im Temperaturbereich von $740^{\circ} \mathrm{C}-1400^{\circ} \mathrm{C}$ an. Eine Erklärung dafür geben A. Atkinson, R.I. Taylor und P.D. Goode [56] nach Interpretationen von Experimenten mit $\mathrm{Ni}^{63}$ - und $\mathrm{O}^{18}$ - Tracern im NiO. Erst bei Temperaturen über $1000^{\circ} \mathrm{C}$ findet demnach die Diffusion der Ni-Ionen im NiO hauptsächlich über Volumendiffusion 
über das Gitter statt. Bei Oxidationstemperaturen von unter $800^{\circ} \mathrm{C}$ findet die sog. „short circuit"-Diffusion (Kurzschlussdiffusion) hauptsächlich über Korngrenzen statt. Die Volumendiffusion trägt nur zu einem sehr geringen Anteil bei, erst ab einer Temperatur von $1000^{\circ} \mathrm{C}$ wird ihr Anteil maßgeblich. Atkinson, Taylor and Goode finden bei NiOSchichten unterhalb einer Dicke von $1 \mu \mathrm{m}$ im Temperaturbereich von $610^{\circ} \mathrm{C}-1000^{\circ} \mathrm{C}$ keinen Transport von $\mathrm{O}_{2}{ }^{18}$ Tracern zur inneren Grenzfläche, sondern lediglich eine Diffusion von $\mathrm{Ni}^{63}$-Tracern zur äußeren Grenzfläche. Durch den Vergleich des hier bestimmten Wachstumsgesetzes und der Aktivierungsenergie mit den Daten aus der Literatur kann gefolgert werden, dass die in dieser Arbeit epitaktisch auf einkristallinen Nickelfilmen gewachsenen NiO-Schichten nach längeren Oxidationszeiten über eine ,short-circuitdiffusion“ im wesentlichen über Korngrenzendiffusion von Nickel zur äußeren Grenzfläche hin wachsen. Die TEM-Aufnahme in Abbildung 5.20 zeigt viele Kleinwinkelkorngrenzen und Versetzungsnetzwerke über die eine „short circuit“ Diffusion stattfinden könnte. Wie bereits erwähnt, wird dieser Mechanismus für sehr dünne NiO-Schichten wahrscheinlich nicht der Vorherrschende sein, da Raumladungszonen und Grenzflächeneffekte hier zusätzlich eine wichtige Rolle spielen.

\subsection{Texturentwicklung in epitaktisch oxidierten NiO-Schichten}

\subsubsection{Die NiO-Anfangsschicht}

Die in den Abbildungen 5.5, 8, 9-11 dargestellten Messungen haben gezeigt, dass es notwendig ist, die Frühstadien der Oxidation, in denen die Textur festgelegt wird, von den späteren Stadien des epitaktischen Wachstums zu unterscheiden. Im folgenden werden daher Schichten im frühen Stadium der Oxidation als Anfangsschichten bezeichnet. Eine direkte Bestimmung der Vorzugsrichtung der Anfangsschichten ist mittels Röntgenbeugung aufgrund der geringen Schichtdicken nicht möglich. Die in Abb. 5.9 dargestellte Messung lässt allerdings aufgrund der sehr viel besseren Texturen des NiO(111)-Anteils den Schluss zu, dass die hier oxidierten Nickelschichten im Frühstadium der Oxidation einen sehr hohen sehr gut texturierten $\mathrm{NiO}(111)$-Anteil gebildet haben. W.D. Wang, N.J. Wu und P.A. Thiel [22] beobachten dagegen mittels LEED, dass oberhalb einer Temperatur von $500 \mathrm{~K}$ eine Nukleation von ausschließich $\mathrm{NiO}(100)$ auf (100) Nickeloberflächen stattfindet. Nur unterhalb von $300 \mathrm{~K}$ finden sie eine Nukleation von $\mathrm{NiO}(111)$. Begründet wird dies damit, dass die $\mathrm{NiO}(100)$-Orientierung aufgrund der geringeren Oberflächenenergie thermodynamisch bevorzugt ist. Diese beträgt für $\mathrm{NiO}(100)$ laut H. Li, F. Czerwinski und J.A. Szunpar [57] 12,6 J/m² und ist damit $40 \%$ geringer als die Oberflächenenergie von $\mathrm{NiO}(111)$, die bei $17,7 \mathrm{~J} / \mathrm{m}^{2}$ liegt. Bei hohen Temperaturen bildet sich somit 
die Orientierung mit der geringeren Oberflächenenergie. Die $\mathrm{NiO}(111)$-Orientierung ist dagegen laut W.D. Wang, N.J. Wu und P.A. Thiel [22] die kinetisch favorisierte Orientierung, da bei der Bildung der ersten Monolagen NiO(111) weniger Ni-Ni-Bindungen aufgebrochen werden müssen als bei der Bildung von $\mathrm{NiO}(100)$. Bei tiefen Temperaturen unter $300 \mathrm{~K}$ ist somit die Kinetik so stark eingefroren, dass sich die $\mathrm{NiO}(100)$-Orientierung nicht mehr bilden kann. Eine Auslagerung einer bei niedrigen Temperaturen hergestellten $\mathrm{NiO}(111)$-Schicht bei $500 \mathrm{~K}$ im Vakuum führt zu einer Umwandlung der $\mathrm{NiO}(111)$ Orientierung in die NiO(100)-Orientierung. D.F. Mitchell, P.B. Sewell und M.Cohen [20] finden mittels RHEED im Temperaturbereich zwischen $40^{\circ} \mathrm{C}-200^{\circ} \mathrm{C}$ Nukleationsschichten auf (100) Nickeloberflächen mit hohen $\mathrm{NiO}(100)$-Anteilen. Allerdings beobachten sie auch immer unabhängig von der Temperatur eine nukleierte $\mathrm{NiO}(111)$-Orientierung. Die eben zitierten Messungen beziehen sich jedoch nur auf eine bzw. sehr wenige Monolagen. Bei dickeren Anfangsschichten spielen zusätzlich die unterschiedlichen Spannungen der verschiedenen Orientierungen eine wichtige Rolle. Die spezifische Spannungsenergie $\rho / \varepsilon$ für die NiO(100)-Orientierung beträgt laut biaxialem Dehnungsmodell $460 \mathrm{GPa}$ und ist damit $25 \%$ geringer als die spezifische Spannungsenergie für die NiO(111)-Orientierung, die bei $615 \mathrm{GPa}$ liegt [58]. Auch hiernach wäre die NiO(100)-Orientierung bevorzugt. Der Flächen-Misfit zwischen $\mathrm{NiO}(100)$ und $\mathrm{Ni}(100)$ liegt aber bei 30\% (vgl. Abb. 4.7) und ist damit sehr hoch, so dass dickere $\mathrm{NiO}(100)$-Schichten vermutlich nicht mehr stabil sind und die NiO(111)-Orientierung, deren Flächen-Misfit zur Ni(100)-Oberfläche nur 19\% beträgt, sich ausbildet. Eine derartige Orientierungsumwandlung von $\mathrm{NiO}(100)$ in $\mathrm{NiO}(111)$ wird von G.A. Carson et al. [23] ab einer Oxidschichtdicke von 3 Monolagen mittels HREELS (High Resolution Electron Energy Loss Spectrum) tatsächlich beobachtet. Anfangsschichten, die mehrere Monolagen dick sind, wachsen also aufgrund der durch den Misfit induzierten Spannungen in der NiO(111) Orientierung auf.

\subsubsection{Die Umwandlungsreaktion}

Im Gegensatz zu den dicken NiO-Schichten, die fast unabhängig von den Oxidationsparametern epitaktisch weiterwachsen, wurde in dünnen NiO-Schichten nach sehr kurzen Oxidationszeiten sowohl bei weiterer Oxidation (Abb. 5.5b, Abb. 5.11) als auch bei der Abkühlung im Vakuum (Abb 5.9, Abb. 5.10) ein Schichtdickenfenster beobachtet, innerhalb dessen eine Orientierungsumwandlung von $\mathrm{NiO}(111)$ in $\mathrm{NiO}(100)$ möglich ist. Es existiert also eine untere kritische Schichtdicke $\mathrm{d}_{1}$ oberhalb der treibende Kräfte für eine derartige „Rekristallisation“ existieren. Die NiO(111)-Reflexe der Filme mit dem fast ausschließlichen $\mathrm{NiO}(111)$-Anteil in Abb. 5.9 zeigen im $\Theta-2 \Theta$ Diffraktogramm eine starke Asym- 
metrie (Abb. 6.2). Diese Asymmetrie bedeutet, dass innerhalb der NiO(111)-Körner ein hoher Spannungsgradient besteht.

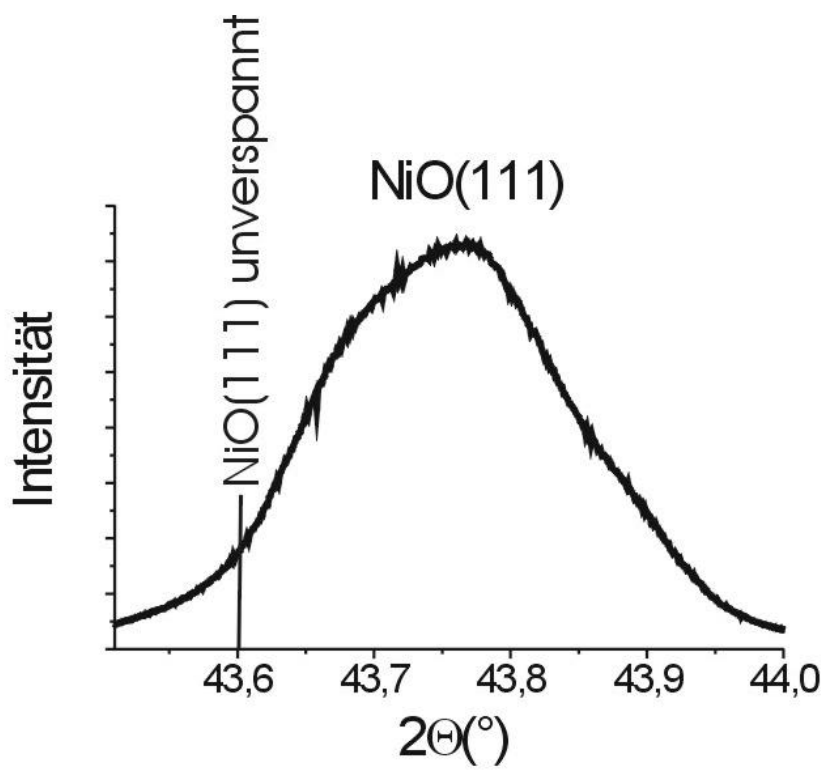

Abb. 6.2 NiO(111)-Reflex der Schichten aus der Abb. 5.9, deren Oxidationszeiten 1, 12 und 20 Minuten betrugen.

Hingegen sind die Reflexe des $\mathrm{NiO}(111)$ - und $\mathrm{NiO}(100)$-Anteils der Schichten, die sich im Vakuum umgewandelt haben, symmetrisch und gegenüber den unverspannten Reflexlagen nicht verschoben. Die hohen Spannungsgradienten im NiO(111) könnten also eine treibende Kraft für die Orientierungsumwandlung in die Orientierung mit der geringsten Oberflächenenergie sein. Da diese Umwandlung aber nur in dünnen Schichten bis zu einer Schichtdicke $\mathrm{d}_{2}$ stattfindet, ist davon auszugehen, dass die Grenzflächenenergie Ni/NiO ebenfalls eine entscheidende Rolle zur Umwandlung von $\mathrm{NiO}(111)$ in $\mathrm{NiO}(100)$ spielt. Die AFM-Bilder (Abb. 5.12 a,b) zeigen, dass bei einer Temperaturbehandlung einer dünnen, glatten NiO-Schicht auf Ni ein beträchtlicher Materialtransport stattfindet, der ähnlich wie bei der Desintegration von Ni/NiO-Multilagen [42] zu einer Strukturänderung der Oberfläche führt. Dieses Experiment ist zwar unter anderen Bedingungen durchgeführt wurden als sie während der eigentlich Oxidation vorherrschen, dennoch zeigt es deutlich den starken Einfluss der Grenzflächenenergie, die auch während der Oxidation eine erhebliche Rolle spielt. Es existiert also ein Schichtdickenbereich $\mathrm{d}_{1}<\mathrm{d}<\mathrm{d}_{2}$, in dem der Spannungsgradient in den $\mathrm{NiO}(111)$-Körnern und die Grenzflächenenergie so hoch ist, dass die NiOSchicht rekristallisiert und die Orientierung mit der geringeren Oberflächenenergie, nämlich die $\mathrm{NiO}(100)$-Körner, sich bildet. Die Abbildungen 5.11 a,b zeigen, dass diese Orientierungsumwandlung auf einer sehr langsamen Zeitskala abläuft. 
Aufgrund des starken Zerfalls dünner glatter NiO-Schichten und den hohen Rauigkeiten der Oxidschichten, ist ein ausgeprägtes Inselwachstum des Oxides sehr wahrscheinlich. Die eben beschriebene Umwandlungsreaktion könnte also folgendermaßen ablaufen. Die $\mathrm{NiO}(111)$-Anfangsschichten wachsen in Form von Inseln auf, die noch nicht zusammegewachsen sind. Ab der unteren kritischen Schichtdicke $d_{1}$ bildet sich aufgrund des hohen Spannungsgradienten in den $\mathrm{NiO}(111)$-Körnern an den Tripelpunkten $\left(\mathrm{Ni}(100) / \mathrm{NiO}(111) / \mathrm{O}_{2}\right)$ die energetisch günstigere $\mathrm{NiO}(100)$-Orientierung (Abb. 6.3).

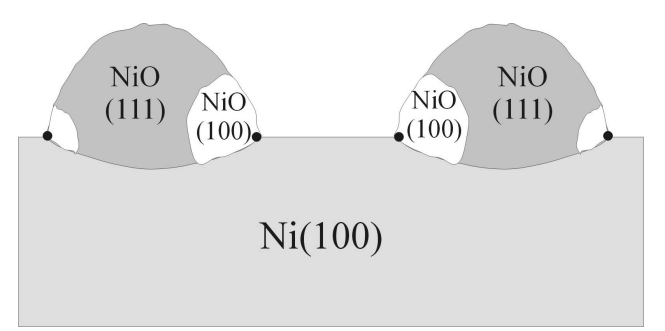

Abb. 6.3 schematische Skizze des Anfangsstadium der epitaktischen Oxidation, wobei die Oxidschichtdicke $d_{1}<d<d_{2}$ ist.

Die Umwandlungsreaktion ist bei einer oberen Schichtdicke $\mathrm{d}_{2}$ abgeschlossen. Möglicherweise sind bei dieser Schichtdicke die NiO-Inseln gerade zusammengewachsen (Abb. 6.4). Da nun keine Tripelpunte mehr zur Verfügung stehen, sind die treibenden Kräfte für eine Umwandlung in die $\mathrm{NiO}(100)$-Orientierung nicht mehr hoch genug.

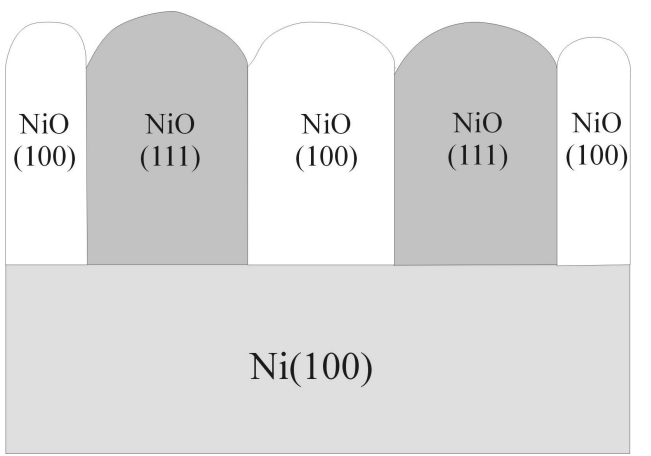

Abb. 6.4 schematische Skizze des Anfangsstadium der epitaktischen Oxidation, wobei die Oxidschichtdicke $d>d_{2}$ ist.

Im nächsten Kapitel wird nun ein Szenario vorgestellt, in dem die Orientierungsumwandlung im wesentlichen für die unterschiedlich orientierten Anteile im $\mathrm{NiO}$ verantwortlich ist und die Temperatur und der Sauerstoffpartialdruck über die Oxidationsrate eingeht. 


\subsubsection{Modellvorstellung zur Texturentwicklung in epitaktisch oxidiertem $\mathrm{NiO}$}

Die Übersichtsdarstellung aller Messungen in Abbildung 5.3 zeigt deutlich den Trend, dass bei niedrigen Temperaturen und Sauerstoffpartialdrücken die NiO-Schicht hauptsächlich aus epitaktisch gewachsenem $\mathrm{NiO}(100)$ besteht, während bei der Verwendung von hohen Temperaturen und Sauerstoffpartialdrücken hauptsächlich epitaktisches $\mathrm{NiO}(111)$ vorliegt. Nicht nur die Temperatur, sondern auch die Sauerstoffpartialdruck bestimmen die Wachstumsgeschwindigkeit der NiO-Schicht (vgl. 6.1.1 Oxidation von Nickel). Ausgehend von den Überlegungen des vorigen Kapitels wird jetzt ein Wachstumsmodell vorgestellt, welches durch zwei charakteristische Schichtdicken gekennzeichnet ist.

\section{Bildung einer $\mathbf{N i O ( 1 1 1 ) - A n f a n g s s c h i c h t ~ s o l a n g e ~} d<d_{1}$}

Aufgrund des geringsten Flächenmisfits bildet sich in der Frühphase der Oxidation eine hauptsächlich (111)-orientierte NiO-Schicht und nicht die aufgrund der geringeren Oberflächenenergie erwartete $\mathrm{NiO}(100)$-Orientierung. Diese ist bis zu einer charakteristischen Schichtdicke $d_{1}$ stabil.

\section{Umwandlungsreaktion von $\mathrm{NiO}(111)$ in $\mathrm{NiO}(100)$ für $d_{1}<d<d_{2}$}

Oberhalb der Schichtdicke $d_{1}$ existiert eine treibende Kraft für die Umwandlungsreaktion, wobei die Spannungen durch Versetzungsbildung reduziert werden und die Orientierung mit der geringeren Oberflächenenergie gebildet wird. Diese treibende Kraft reicht ab einer Dicke $d>d_{2}$ nicht mehr aus um die Umwandlungsreaktion zu aktivieren.

\section{Epitaktisches Wachstum für $d>d_{2}$}

Beim weiteren epitaktischen Wachsen bleiben sowohl die Güte der Textur als auch die Volumenanteile der verschieden orientierten Körner konstant.

Je nach Oxidationsparametern befindet sich die NiO-Schicht lange bzw. kurz in der Wachstumsphase 2, d.h. im Bereich der Schichtdicke $d_{1}<d<d_{2}$. Werden niedrige Werte der Oxidationsparametern verwendet, so wächst die Schicht sehr langsam und sehr viel $\mathrm{NiO}(100)$ ensteht während der Umwandlungsreaktion. Je höher die Werte der Oxidationsparameter gewählt werden, desto weniger Zeit bleibt für die Umwandlungsreaktion und desto mehr $\mathrm{NiO}(111)$ verbleibt in der Schicht, so dass bei sehr hohen Werten der Oxidationsparameter ausschließlich $\mathrm{NiO}(111)$ entsteht. Das Verhältnis aus Oxidationskonstanten und Umwandlungszeit bestimmt demnach den $\mathrm{NiO}(100)$ und $\mathrm{NiO}(111)$-Anteil in der NiO-Schicht. In den Abbildung 6.5-6.7 ist das epitaktische Wachstum von $\mathrm{NiO}$ auf 
Ni(100) für verschieden hohe Werte der Oxidationsparameter schematisch dargestellt. Da in diesen Zeichnungen nur die Entwicklung der verschieden orientierten Anteile dargestellt werden soll, wurden zur Vereinfachung die Grenzflächen glatt gezeichnet. In Abbildung 6.5 wurden sehr niedrige Werte der Oxidationsparameter gewählt, so dass die Umwandlungsreaktion schon bei einer Schichtdicke, die kleiner als $d_{2}$ ist, abgeschlossen ist. Dieses dargestellte Bild erklärt die zeitaufgelöste Messung 5.5a. In dieser liegt von Beginn der Messung an der $\mathrm{NiO}(100)$-Anteil bei ungefähr 90\%, d.h. die Umwandlungsreaktion ist schon beendet, bevor die Schicht so dick ist, dass sie im Röntgendiffraktogramm beobachtet werden kann. In Abbildung 6.6 ist das Oxidwachstum für etwas höhere Werte der Oxidationsparameter dargestellt. Hier ist die Schichtdicke $\mathrm{d}_{2}$ erreicht, bevor die Umwandlungsreaktion beendet ist. Die in Abbildung 5.5b dargestellte Messung zeigt eine solche Umwandlung in der Anfangsphase der Oxidation, während danach die NiO-Anteile konstant bleiben. In Abbildung 6.7 ist das Wachstum einer NiO-Schicht bei sehr hohen Werten der Oxidationsparameter dargestellt. Das Schichtwachstum ist nun relativ zur Umwandlungsreaktion so schnell, dass die Schichtdicke $\mathrm{d}_{2}$ bereits erreicht ist, nachdem sich erst ein sehr geringer Bruchteil des $\mathrm{NiO}(111)$ zu $\mathrm{NiO}(100)$ umgewandelt hat.

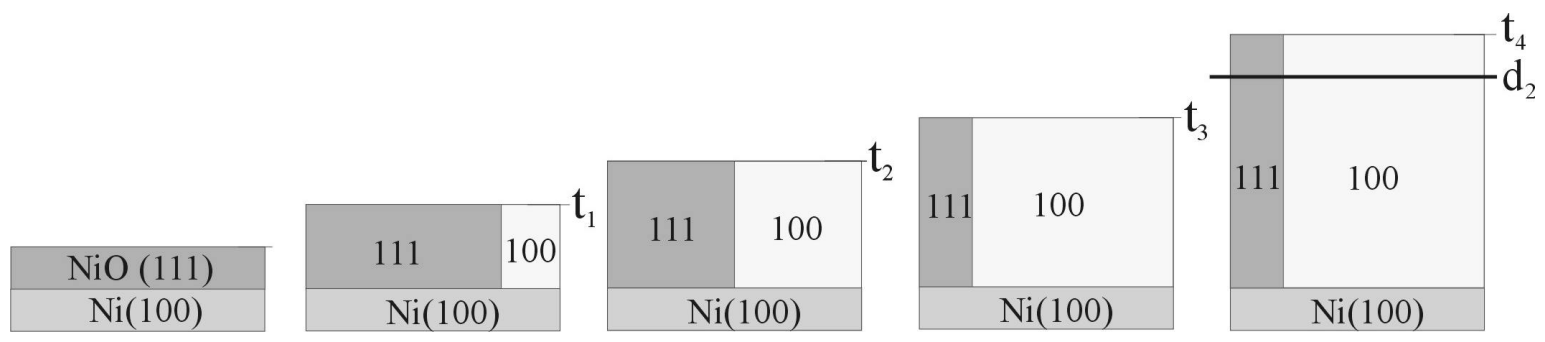

Abb. 6.5 Epitaktisches Wachstum von NiO auf einkristallinem Nickel bei sehr niedriger Temperatur und niedrigem Sauerstoffpartialdruck, d.h. die Wachstumsgeschwindigkeit ist sehr langsam. Die Schichtdicke $d_{2}$ ist die Schichtdicke, bis zu der eine Umwandlungsreaktion stattfinden kann.

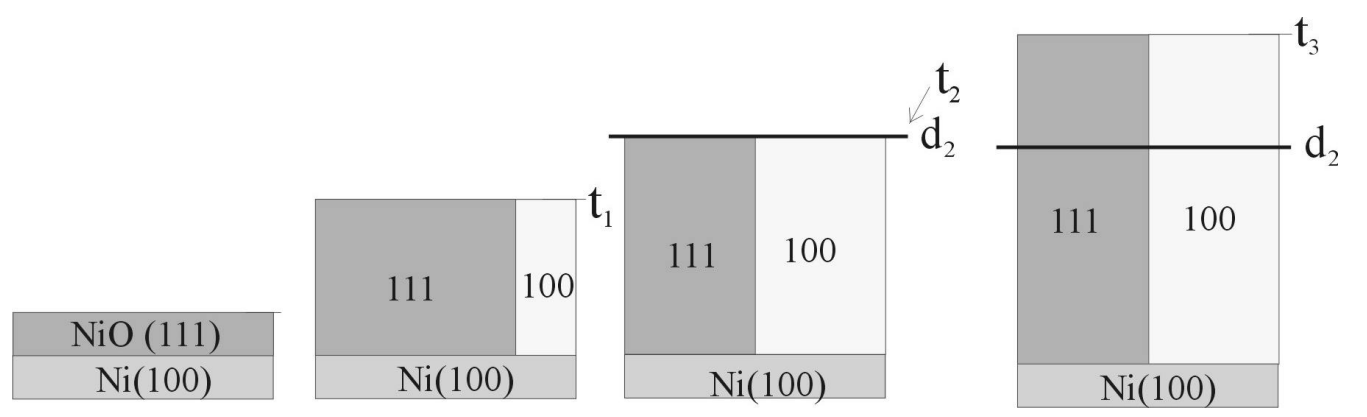

Abb. 6.6 Epitaktisches Wachstum von NiO auf einkristallinem Nickel bei etwas höherer Temperatur und höherem Sauerstoffpartialdruck, d.h. die Wachstumsgeschwindigkeit ist schneller als die in Abb. 6.3. Die Schichtdicke $d_{2}$ ist die Schichtdicke, bis zu der eine Umwandlungsreaktion stattfinden kann. 


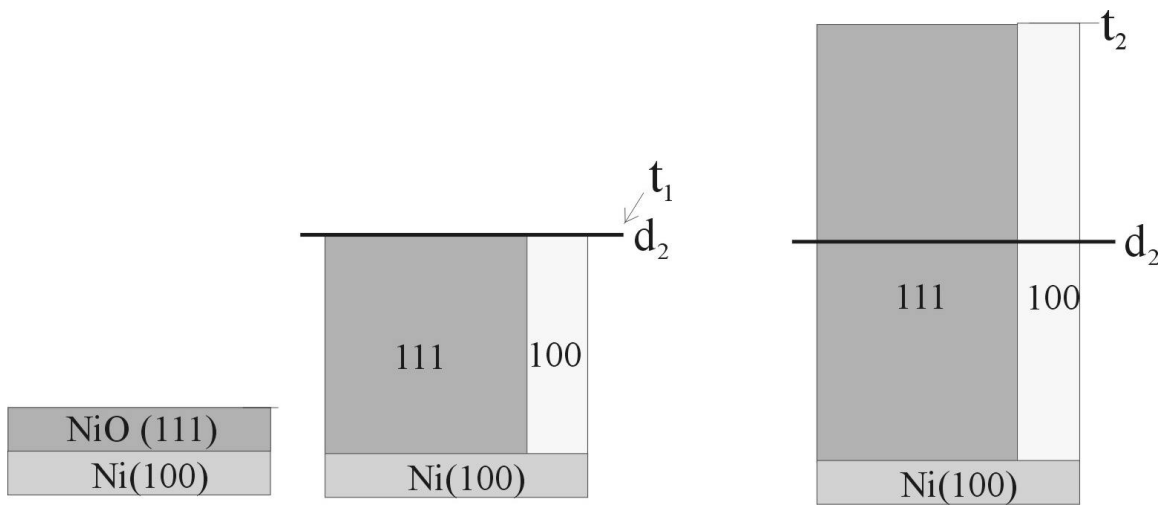

Abb. 6.7 Epitaktisches Wachstum von NiO auf einkristallinem Nickel bei hohen Temperaturen und hohem Sauerstoffpartialdruck, d.h. die Wachstumsgeschwindigkeit ist noch schneller als die in Abb. 6.4. Die Schichtdicke $d_{2}$ ist die Schichtdicke, bis zu der eine Umwandlungsreaktion stattfinden kann.

\subsubsection{Texturentwicklung im NiO bei der Oxidation von $\{100\}<100>$ $\mathrm{Ni}_{87} \mathrm{Cr}_{13}$}

Nach dem im vorigen Kapitel vorgestellten Modell ist zu erwarten, dass ein Zusatz, der die Oxidationsgeschwindigkeit reduziert, den Anteil von $\mathrm{NiO}(100)$ erhöht. Die „cross over“Linie in Abb 5.3 sollte sich also zu höheren Temperaturen und Drücken verschieben. Experimentell gibt es bei der Oxidation der NiCr-Schichten keinen Hinweis auf eine Umwandlungsreaktion von $\mathrm{NiO}(111) \mathrm{zu} \mathrm{NiO}(100)$. Allerdings konnten die Oxidationen der NiCr-Legierung wie bereits angesprochen nicht in-situ im Röntgendiffraktometer durchgeführt werden. Wie bereits beschrieben bildet, sich bei der Oxidation von $\mathrm{Ni}_{87} \mathrm{Cr}_{13}$ zuerst das $\mathrm{NiO}$, welches zum späteren Zeitpunkt der Oxidation durch eine selektive Oxidation des Chroms an der inneren Grenzfläche unterwachsen wird. Nun ist es auch hier möglich, dass sich zunächst eine $\mathrm{NiO}(111)$-Anfangsschicht epitaktisch bildet. Die nun beginnende selektive Oxidation des Chroms findet hauptsächlich an den Korngrenzen statt [35]. Da das Wachstum der NiO-Schicht hauptsächlich durch Materialtransport durch die Korngrenzen bestimmt wird, bewirkt die einsetzende innere Oxidation eine starke Reduzierung der Oxidationsgeschwindigkeit, da die Chromoxide sehr kompakt sind und die Beweglichkeit des Nickel stark einschränkt. Das nun langsame Weiterwachsen der NiO-Schicht ermöglicht eine lange Zeit für die Orientierungsumwandlung in $\mathrm{NiO}(100)$. Bei der Oxidation von $\mathrm{NiCr}$ bedeuten also hohe Oxidationsparameter nicht ein schnelles Wachstum der NiOSchicht. So wird sowohl bei den oxidierten RABiTS-Bändern als auch bei den oxidierten einkristallinen Filmen unabhängig von der Temperatur bei hinreichend hohen Drücken praktisch nur (100) orientiertes $\mathrm{NiO}$ beobachtet. Allerdings zeigen auch beide Systeme, dass der NiO(100)-Anteil signifikant mit dem Sauerstoffpartialdruck zunimmt (Abb 5.4b). Die Zunahme des $\mathrm{NiO}(111)$-Anteils mit abnehmenden Druck kann folgendermaßen erklärt 
werden. Da zu niedrigen Sauerstoffpartialdrücken der Anteil an Nickelchromoxiden und Chromoxiden steigt, ist es möglich, dass sich schon im frühen Stadium der Oxidation kompakte Nickelchromoxid- oder Chromoxidschichten zwischen Legierung und der NiOSchicht gebildet haben, die die Spannungen im NiO so weit reduzieren, dass die treibenden Kräfte für eine weitere Umwandlungsreaktion nicht mehr ausreichen. Zusammengefasst muss hier festgestellt werden, dass die Oxidation von Legierungen ein zu komplexer Vorgang ist, als dass erwartet werden könnte, dass ein Modell für reines Nickel direkt übertragbar wäre.

\subsubsection{Einfluss weiterer Parameter auf die Oxidation}

Wie die Übersichtsdarstellung der Messungen in Abbildung 5.3 zeigt, ist das Verhältnis des $\mathrm{NiO}(100)$ und des $\mathrm{NiO}(111)$-Anteil schlecht reproduzierbar und mit großen Fehlerbalken behaftet. Diese schlechte Reproduzierbarkeit wurde auch von anderen Gruppe festgestellt u.a. von D.F. Mitchell, P.B. Sewell und M. Cohen [20], die die Anfangsstadien der Oxidation von Ni(100)-Oberflächen mittels RHEED beobachteten. Die folgenden nun aufgeführten Punkte scheinen eine für die Reproduzierbarkeit wesentliche Rolle zu spielen. O. Kubaschewski, O. von Goldbeck [59] und E.A. Gulbransen und K.F. Andrew [60] führten Versuche zur Kinetik der Oxidation von hochreinem Nickel durch und stellten fest, dass je nach verwendetem Ausgangsmaterial die Zunderkonstanten bis zu 4 Zehnerpotenzen voneinander abwichen, obwohl die Nickelproben jeweils reiner als 99,9\% waren. Grund dafür ist die starke Abhängigkeit der Nickelleerstellenkonzentration im $\mathrm{NiO}$ von geringen Zusätzen. Da der geschwindigkeitsbestimmende Faktor der Oxidation die Diffusion von Nickelionen über Nickelleerstellen durch die Oxidschicht ist, ändert sich die Oxidationsgeschwindigkeit ebenfalls erheblich mit Leerstellenkonzentration. Die Wachstumskinetik ist also nicht nur durch die Oxidationsparameter Temperatur und Sauerstoffpartialdruck, sondern ebenfalls durch kleinste Verunreinigungen in dem Nickelfilm bestimmt. In dem im vorigen Kapitel vorgestellten Modell hängt die Texturausbildung stark von der Oxidationsrate ab. Es wundert daher nicht, dass wie in dieser Arbeit dargestellt, die Oxidation in unterschiedlichen Anlagen nur zu bedingt vergleichbaren Ergebnissen führt. Reproduzierbare Systematiken wurden nur beobachtet, wenn die Ni-Ausgangsfilme unter identischen Bedingungen (insbesondere mit dem gleichen Target) hergestellt wurde und die gleiche Anlage für die Oxidation verwendet wurde. Von dieser Problematik unbeeinflusst sind aber die generellen Trends. So findet man z.B. stets ein NiO(111)-Wachstum, wenn der Druck und die Temperatur hinreichend hoch sind. In diesem Sinn ist das in Abbildung 5.3 gezeigte Orientierungsdiagramm repräsentativ, nur die eingezeichnete „Cross Over“-Linie, die den Bereich des $\mathrm{NiO}(100)$ und $\mathrm{NiO}(111)$-Wachstums trennt, verschiebt sich mit den 
exakten Oxidationsbedingungen. Die geschilderten Gründen führen wahrscheinlich auch dazu, dass die in den Abbildungen 5.19 und 5.20 dargestellten „quadratischen Löcherstrukturen " nicht systematisch reproduzierbar waren. Dies unterstreicht die Wichtigkeit, den Einfluss einzelner Parameter voneinander zu trennen um Verfahrensvorschriften für die stabile Oxidation von Nickel- und Nickellegierungssoberflächen zu erarbeiten und wird insbesondere für den Transfer der Forschungsergebnisse nach weiterer Optimierung hinsichtlich der Entwicklung von Oxidationsanlagen Bedeutung erlangen. Ein anderer wichtiger Punkt für die Reproduzierbarkeit ist die Oberflächenbeschaffenheit des einkristallinen Nickelfilms. So beobachtet C. Cantoni [61] z.B. mittels RHEED, dass sich auf einigen Ni $\{100\}<100>$ RABiTS-Bändern Schwefel c $(2 \times 2)$ Überstrukturen bilden. Diese Überstrukturen ermöglichen erst das epitaktische Wachstum von YSZ auf diesen Bändern. Auf Ni-RABiTS-Bändern, die diese Strukturen nicht aufweisen, wächst der YSZ-Film nicht ausschließlich in (100)-Richtung. Die Bildung von Überstrukturen von VerunreinigungsAd-Atomen können auch auf das Anfangsstadium der epitaktischen Oxidation einen erheblichen Einfluss haben.

\subsection{Entwicklung der Oberflächenmorphologie}

\subsubsection{Einfluss der Substratrauigkeit}

Im Kapitel 4 „Oxidation der RABiTS-Bänder bei hohen Temperaturen“ wurde der Einfluss der Substratrauigkeit auf die Oxidrauigkeit untersucht. Es wurde gezeigt, dass zwischen der Oxid- und der Substratrauigkeit eine lineare Abhängigkeit besteht, wobei die Differenz aus Oxid- und Substratrauigkeit nahezu konstant ist. Dies lässt auf eine substratunabhänige Rauigkeitsentwicklung schliessen, die im folgenden diskutiert wird.

\subsubsection{Frühstadien der Rauigkeit}

Die bei $500^{\circ} \mathrm{C}$ im Vakuum ausgelagerte $10 \mathrm{~nm}$-dicke gesputterte NiO-Schicht (Abb. 5.12b) zeigt eine mittlere Rautiefe von ungefähr $8 \mathrm{~nm}$, dieser Wert entspricht fast der ursprünglichen Schichtdicke. Die Entstehung der Anfangsrauigkeit des NiO ist also nicht nur durch die Prozesse bei der eigentlichen Oxidbildung bestimmt. Dieses Aufrauen dünner und glatter NiO-Schichten ist bereits aus Untersuchungen zur Desintegration von Ni/NiOMultilagen bekannt. S. Dreyer [42] beobachtete, dass Vielfachschichten aus $100(\mathrm{Ni}(2 \mathrm{~nm}) / \mathrm{NiO}(6 \mathrm{~nm}))$ Doppellagen bei einer Auslagerungstemperatur von $550^{\circ} \mathrm{C}$ in eine körnerartige Struktur mit Partikelgrößen um die $11 \mathrm{~nm}$ zerfallen. Die treibenden 
Kräfte einer solchen Desintegration sind die Grenzflächenenergien zwischen den einzelnen Lagen. Wie das in Abb 5.12 dargestellte Experiment zeigt, scheint bereits die Energie einer Grenzfläche zwischen Nickel und Nickeloxid bei einer Temperatur von $500^{\circ} \mathrm{C}$ auszureichen, um einen solchen Zerfall der Schichtstruktur auszulösen. Die Grenzflächenenergien verursachen also bereits zu Beginn der Oxidation eine sehr hohe Initialrauigkeit. Oxidwachstum und Desintegration finden dabei bei Oxidationstemperaturen $>500^{\circ} \mathrm{C}$ wahrscheinlich parallel statt, so dass es zu einer Art Inselwachstum kommt. Neben dem Zerfall von homogenen Oxidschichtlagen tragen mit Sicherheit auch andere Prozesse zur Rauigkeitsentwicklung bei. Verunreinigungen im Nickel können lokal die Bildungenthalpie des Nickeloxids herabsetzen und Adsorbate können die Oxidation behindern. Beides führt zu einer inhomogenen Keimbildung. Im folgenden Kapitel wird untersucht wie sich die Initialrauigkeit im Verlauf der Oxidation weiterentwickelt.

\subsubsection{Entwicklung der Rauigkeit}

Wie bereits bei den Messungen der Rauigkeiten beschrieben wurde, ist eine direkte Messung der NiO-Schichtdicken nicht möglich. Um dennoch ein Wachstumsgesetz für die Rautiefe in Abhängigkeit von der Schichtdicke zu ermitteln, wurde zum einen die mittlere Rautiefe nach verschiedenen Oxidationszeiten (Abb. 5.17a) und zum anderen bei verschiedenen Oxidationstemperaturen (Abb. 5.17b) bei sonst identischen Oxidationsparametern gemessen. Die Rautiefen der bei verschiedenen Oxidationszeiten oxidierten Ni-Schichten sind sehr gering und liegen deutlich unter denen, der bei unterschiedlichen Temperaturen oxidierten. Grund dafür sind höchstwahrscheinlich die unterschiedlichen Oxidschichtdikken. Die beiden unterschiedlichen Messungen wurden mit Nickelschichten durchführt, die mit unterschiedlichen Ni-Targets hergestellt wurden. Wie bereits im Kapitel 6.3.5 „Einfluss anderer Parameter" erwähnt, können die verschiedenen Ni-Targets einen erheblichen Einfluss auf die Oxidationsrate haben. Es kann also davon ausgegangen werden, dass die Messungen der Rauigkeiten bei verschiedenen Zeiten die Rauigkeitsentwicklung in eher dünneren Oxidschichten beschreibt, während die Messungen der Rautiefen bei unterschiedlichen Temperaturen eher die Rauigkeitsentwicklung in dickeren Oxidschichten wiedergibt. Die Rautiefenmessung in Abhängigkeit von der Oxidationszeit zeigt ungefähr eine lineare Abhängigkeit (Abb. 5.17a). Da für das Oxidschichtwachstum das parabolische Wachstumsgesetz gilt (Abb. 5.7), folgt dass die Rauigkeit proportional zur Schichtdicke zum Quadrat ist $\mathrm{R}_{\mathrm{z}} \sim \mathrm{t} \sim \mathrm{d}^{2}$.

Die Rautiefenmessungen bei verschiedenen Temperaturen spiegelt eher die Rauigkeitsentwicklung in dickeren NiO-Schichten wieder. F. Czerwinski und J.A. Szpunar [62] beobachteten, dass die Rauigkeit in dem hier betrachteten Temperaturbereich $\left(350^{\circ} \mathrm{C}-700^{\circ} \mathrm{C}\right)$ 
nur sehr schwach von der Temperatur abhängt, so dass die in Auftragung 5.17b dargestellten Temperaturen in Schichtdickenverhältnisse folgendermaßen umgerechnet werden können. Aus dem parabolischen Wachstumsgesetz

$\mathrm{d} \sim\left(\mathrm{k}_{\mathrm{p}} \mathrm{t}\right)^{1 / 2}$

mit d: Schichtdicke, $\mathrm{k}_{\mathrm{p}}$ : parabolische Zunderkonstante, $\mathrm{t}$ : Zeit

folgt mit

$\mathrm{k}_{\mathrm{p}}=\mathrm{k}_{0} \exp \left(-\mathrm{E}_{\mathrm{A}} /\left(\mathrm{k}_{\mathrm{B}} \mathrm{T}\right)\right)$,

bei konstantem $\mathrm{k}_{0}$ und $\mathrm{t}$, und unter Benutzung der ermittelten Aktivierungsenergie $\mathrm{E}_{\mathrm{A}}=1,8 \mathrm{eV}=174 \mathrm{~kJ} / \mathrm{mol}$

$\mathrm{d} \sim\left[\exp \left(-\mathrm{E}_{\mathrm{A}} /\left(\mathrm{k}_{\mathrm{B}} \mathrm{T}\right)\right)\right]^{1 / 2}$.

In der Abbildung 6.8 wurde die mittlere Rautiefe $\mathrm{R}_{\mathrm{z}}$ gegen $\left[\exp \left(-\mathrm{E}_{\mathrm{A}} /\left(\mathrm{k}_{\mathrm{B}} \mathrm{T}\right)\right)\right]^{1 / 2}$ doppeltlogarithmisch aufgetragen. Der lineare Fit ergibt, dass die mittlere Rautiefe ungefähr mit der Wurzel der Schichtdicke wächst, denn die Geradensteigung beträgt 0,53(9).

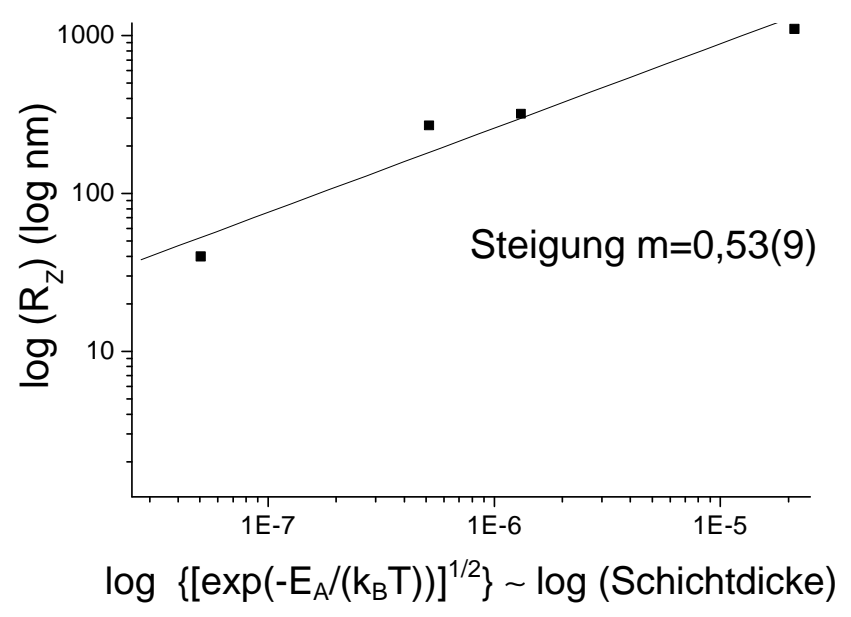

Abb. 6.8 Mittlere Rautiefe $R_{\mathrm{z}}$ doppelt-logarithmisch aufgetragen gegen die Schichtdicke $\left(\sim\left[\exp \left(-E_{A} /\left(k_{B} T\right)\right)\right]^{1 / 2}\right)$. Die Messwerte wurden aus der Abbildung 5.17b entnommen.

Die Rauigkeitszunahme wird also mit zunehmender Schichtdicke schwächer, während sie bei dünnen Oxidschichten noch mit dem Quadrat der Schichtdicke steigt, nimmt sie bei größeren Schichtdicken nur noch mit der Wurzel der Schichtdicke zu. Dieses Ergebnis stimmt qualitativ gut mit den Messungen von F. Czerwinski und J.A. Szpunar [62] überein, die ebenfalls eine starke Rauigkeitszunahme bei dünnen Oxidschichten und eine immer schwächer werdende bei dickeren Oxidschichten beobachten. 
Da die hohe Rauigkeit das epitaktische Abscheiden weiterer Schichten verhindert, muss diese stark reduziert werden. Eine Möglichkeit sowohl die Rauigkeit zu minimieren als auch die Texturen zu verbessern haben F. Czerwinski und J.A. Szpunar [62] gefunden. Sie schieden auf einem (100) Nickeleinkristall eine 14 nm dicke $\mathrm{CeO}_{2}-\mathrm{Schicht}$ ab. Bei der anschließenden Oxidation bildet sich das $\mathrm{NiO}$ sowohl an der inneren als auch an der äußeren Grenzfläche. Die so entstehende NiO-Schicht ist besser texturiert und weist erheblich geringere Rauigkeiten auf, als der oxidierte Nickeleinkristall ohne $\mathrm{CeO}_{2}$-Schicht. Andere Gruppen minimieren die Rauigkeit der NiO-Schicht, indem sie $\mathrm{MgO}$ auf die NiO-Schicht aufbringen. Die MgO-Abscheidung oder die Abscheidung anderer Pufferschichten in der hier verwendeten Ionenstrahlsputteranlage hat allerdings nicht zu einer Rauigkeitsminimierung geführt. 


\section{Zusammenfassung}

In der vorliegenden Arbeit wurde die Oxidation von Substraten für die Herstellung von HTSL-Bandleitern unter zwei verschiedenen Gesichtspunkten betrachtet. Im Kapitel 3 „Stabilität von Substrat-Puffer-Systemen“ wurde untersucht, welche Anforderungen das Schichtsystem aus Pufferschicht und Substrat bei der Deposition von YBaCuO in einem kontinuierlichen Herstellungsprozess erfüllen muss. Dabei wurde festgestellt, dass die Verwendung von oxidationsbeständigen Substraten unabdingbar ist, da selbst durch gut texturierte dickere Pufferschichten der Sauerstofftransport durch gestörte Bereiche dieser Schicht dazu führt, dass oxidationsunbeständige Materialien wie z.B Ni und $\mathrm{NiCu}$ an diesen Stellen partiell oxidieren. Diese Bereiche können zu einem Ablösen des Bandes vom Substrat führen. Bei der Verwendung von Ni-Legierung erfüllt die nichtmagnetische $\mathrm{Ni}_{84} \mathrm{Cr}_{16}$-Legierung weitgehend dieses Kriterium.

Da eine Oxidation des Substrates während der $\mathrm{YBaCuO}$-Deposition aufgrund der hohen Kondensationsbedingungen $\left(\mathrm{T} \approx 740^{\circ} \mathrm{C}, \mathrm{p} \approx 0,6 \mathrm{mbar} \mathrm{O}_{2}\right.$ ) nicht vollständig vermieden werden kann, wurde im Kapitel 4 „Oxidation von RABiTS-Bänder unter hohen Temperaturen“ die gezielte epitaktische Oxidation untersucht. Das NiO soll dann entweder selber als Pufferschicht bzw. als Unterlage für die Abscheidung weiterer Pufferschichten wirken. Dabei wurde festgestellt, dass es mittels einfacher Methoden möglich ist, $\mathrm{Ni}, \mathrm{NiCr}$ und $\mathrm{NiV}$ RABiTS-Bänder so zu oxidieren, dass sich das Eigenoxid epitaktisch bildet. Allerdings bildet sich nicht nur die gewünschte $\mathrm{NiO}(100)$-Textur, sondern ebenfalls eine $\mathrm{NiO}(111)$ Textur. Durch geeignete Wahl der Oxidationsparameter kann die unerwünschte NiO(111)Textur bei der Oxidation von NiCr-RABiTS-Bändern vollständig unterdrückt werden, wobei bei der Oxidation von Ni und NiV-RABiTS-Bändern immer ein kleiner NiO(111)Anteil beobachtet wurde. Der NiO(100)-Anteil kann innerhalb der Ebene in der $0^{\circ}$ oder der $45^{\circ}$-Epitaxiebeziehung aufwachsen. Die Relation lässt sich über den Sauerstoffpartialdruck einstellen. Die Oberflächen der oxidierten Ni und NiCr-Bänder bestehen aussschließlich aus $\mathrm{NiO}$, die Oberflächen der oxidierten NiV-Bänder zeigen zusätzlich vanadiumreiche Ausscheidungen. Von den hochtemperaturoxidierten Bändern sind also nur NiCr und eingeschränkt Ni als Puffer geeignet. Allerdings verhindern hier die großen Rauigkeiten die epitaktische Abscheidung weiterer Puffer- oder HTSL-Schichten. Schwerpunkt der Arbeit war es, den Mechanismus und die Texturentwicklung in auf (100)-Nickeloberflächen oxdierten NiO zu verstehen. Daher wurden im Kapitel 5 „Epitaktische Oxidation einkristalli- 
ner $\mathrm{Ni}$ - und NiCr-Filme" an auf Einkristallen epitaktisch abgeschiedenen quasieinkristallinen Filmen gezielte Untersuchung zur Texturentwicklung durchgeführt. In dem in dieser Arbeit gewählten Parameterbereich kann die Texturbildung während der Oxidation von einkristallinem $\{100\}<100>$ Nickels mittels folgendem Modell beschrieben werden.

Aufgrund des geringsten Flächen-Misfits bildet sich in der Frühphase der Oxidation eine hauptsächlich (111)-orientierte NiO-Schicht und nicht die aufgrund der geringeren Oberflächenenergie erwartete $\mathrm{NiO}(100)$-Orientierung. Diese ist bis zu einer charakteristischen Schichtdicke $\mathrm{d}_{1}$ stabil.

Oberhalb der Schichtdicke $d_{1}$ existiert eine treibende Kraft für die Umwandlungsreaktion von $\mathrm{NiO}(111)$ zu $\mathrm{NiO}(100)$, wobei die Spannungen durch Versetzungsbildung reduziert werden und die Orientierung mit der geringeren Oberflächenenergie gebildet wird. Diese treibende Kraft verschwindet in dicken Oxidlagen charakterisiert durch eine zweite Dicke $\mathrm{d}_{2}$. Oberhalb der zweiten charakterisitischen Schichtedicke findet lediglich weiteres epitaktischen Wachsen über Korngrenzendiffusion statt. Dabei bleiben sowohl die Güte der Textur als auch die Volumenanteile der verschieden orientierten Körner konstant. Die sich letztendlich ausbildende Textur des NiO hängt deshalb vom Verhältnis der Zeit der Umwandlungsreaktion zur Oxidationsrate ab. Bei niedriger Oxidationsrate bildet sich also hauptsächlich (100)-orientiertes NiO, während sich bei hohen Oxidationsraten hauptsächlich (111)-orientiertes $\mathrm{NiO}$ bildet. Dieses Modell zeigt sehr deutlich, dass die letztendlich entstehende Textur empfindlich von den Bedingungen in den Frühstadien der Oxidation abhängt, was eine stabile Prozessführung erheblich erschwert.

Sowohl die oxidierten RABiTS-Bänder als auch die oxidierten einkristallinen Filme zeigen eine ausgeprägte Oberflächenmorphologie. Die hohen Rauigkeiten werden bereits im Anfangsstadium der Oxidation durch die hohen Grenzflächenenergien zwischen Nickel und Nickeloxid initiiert. Die eigentliche Rauigkeitsentwicklung ist substratunabhängig. Die $\mathrm{R}_{\mathrm{z}^{-}}$ Rauigkeit nimmt zu Beginn der Oxidation ungefähr mit dem Quadrat der Oxidschichtdicke zu. Diese Abhängigkeit wird zu dickeren Oxidschichten immer schwächer und mündet in eine Abhängigkeit zur Wurzel der Schichtdicke. 


\section{Literaturverzeichnis}

[1] Bericht zur Supraleitung aus Jugend forscht, (1997)

[2] H. Kamerlingh Onnes, Comm. Leiden 120b, (1911)

[3] J.G. Bednorz, K.A. Müller, Z. Phys. B 64, 189, (1986)

[4] F.C. Moon, P.-Z. Chang, Appl. Phys. Lett. 52 (1988), 1534

[5] F.C. Moon, P.-Z. Chang, Appl. Phys. Lett. 56 (1990), 397

[6] H.J. Bornemann et al, Advances in Superconductivity IV, Tokyo (1994)

[7] M. K. Wu, J.R. Ashburn, C.J. Torng et al, Phys. Rev. Lett. 58 (1987), 908

[8] D. Dimos, P. Chaudhari, J. Mannhart, Physical Review B, 41,(1990), 4038

[9] S. Jin, T.H. Tiefel, R.C. Sherwood et al, Appl. Phys. Lett. 52 (1988), 2074

[10] S. Jin, T.H. Tiefel, R.C. Sherwood et al, Phys. Rev. B 37 (1988), 7850

[11] D.H.A. Blank, D.J. Adelerhof, J. Flokstra, H. Rogalla, Physica C 167, 423 (1990)

[12] W. Decker, L. Klippe, A. Nürnberg, R. Stolle, O. Thomas, Applied Superconductivity, 407, (1993)

[13] Q.X. Jia, W.A. Anderson, Applied Physics Letters 57, 304 (1990)

[14] Y. Iijima, N. Tanabe, Y. Ikeno, O. Kohno, Physica C, 185, (1991), 1959

[15] A. Goyal, D.P. Norton, J.D. Budai, M. Paranthaman, E.D. Specht, D.M. Kroeger, D.K. Christen, Q. He, B. Saffian, F. A. List, D.F. Lee, P.M. Martin, C.E. Klabunde, E. Hartfield, V.K. Sikka, Applied Physics Letters, 69 (1996), 1795

[16] K. Matsumoto, S. Kim, J.-G. Wen, I. Hirabayashi, T. Watanabe, N. Uno, M. Ikeda, IEEE Transactions on Applied Superconductivity, vol. 9, No. 2, (1999)

[17] A. Ginsbach, R. Schneider, H.W. Grueninger, G. Grabe, IEEE Transactions on Magnetics, vol. 27, no. 2, (1991)

[18] Carl Wagner, Journal of the electrochemical Society, vol 99, No 10, (1952)

[19] P.H. Holloway, J.B. Hudson, Surface Science 43, (1974), 123-140

[20] D.F. Mitchell, P.B. Sewell, M. Cohen, Surface Science 61, (1976), 355-376 
[21] R.G. Smeenk, R.M. Tromp, J.W.M. Frenken, F.W. Saris, Surface Science 112 (1981) 261-271

[22] W.D. Wang, N.J. Wu, P.A. Thiel, J. Chem. Phys. 92(3), 1990

[23] G.A. Carson, M.H. Nassir, K.W. Wulser, M.A. Langell, Surface Science Spectra, Vol. $5,(1998)$

[24] H.U. Krebs, Strukturuntersuchungen an amorphen und kristallinen (Zr,Hf)-FeLegierungen, Dissertation, Institut für Metallphysik, Universität Göttingen (1984)

[25] J. Dzick, Mechanismen der ionenstrahlunterstützten Texturbildung in YSZ-Filmen, Dissertation, Institut für Materialphysik, Universität Göttingen (2000)

[26] J. Wiesmann, Wachstum von biaxial texturierten YSZ-Dünnfilmen mittels ionenstrahlunterstützter Deposition, Dissertation, Institut für Materialphysik, Universität Göttingen (1998)

[27] L.O.Kautschor, Dissertation in Vorbereitung, Institut für Materialphysik, Universität Göttingen (2002)

[28] J. Hoffmann, Präparation und Untersuchungen der supraleitenden Eigenschaften von metastabilen NbAl-Dünnfilmen, Diplomarbeit, Institut für Metallphysik, Universität Göttingen(1991)

[29] H.R. Kaufmann, Advances in Electronics and Electron Physics 36, Hrsg L. Marton, (1974) S.265

[30] J. Wiesmann, Herstellung und Charakterisierung von texturierten $\mathrm{CeO}_{2^{-}}$und YSZ Pufferschichten auf technische Substraten, Diplomarbeit, Institut für Metallphysik, Universität Göttingen (1994)

[31] American Institute of Physics Handbook (1972)

[32] R. Stubican, R.C. Hink, S.P. Ray, Journal of the American Ceramic Society, 61, (1978), 17

[33] F. Hund, Zeitschrift für Elektrochemie, 55, (1951), 363

[34] F.G. Moreno, Laserdeposition von HTSL-Filmen auf Langsubstraten und der Einfluss mechanischer Spannungen, Dissertation, Institut für Materialphysik, Göttingen (1999)

[35] K. Hauffe, Oxidation von Metallen und Legierungen, Springer Verlag, Berlin Göttingen Heidelberg, (1956)

[36] Thomas Simon, Augerelektronentiefenprofilanalysen von hochtemperatursupraleitenden YBaCuO-Filmen auf metallischen und oxidischen Substraten, Dissertation, Institut für Metallphysik, Universität Göttingen, 1995 
[37] N.B. Pilling, R.E. Bedworth, Ind. Eng. Chem., 17 (1925)

[38] C. Wagner, J. electrochem. Soc., 108 (1961), 954

[39] J.A. Sartell et al, Trans. Am. Soc. Metals, 50 (1958), 1047

[40] R.L. Levin, J.B. Wagner, J. electrochem. Soc., 108 (1961), 954

[41] K.Thiele, Korngrenzen in HTSL durch epitaktische Abscheidungen auf Bikristallsubstraten, Diplomarbeit, Institut für Materialphysik, Universität Göttingen (1999)

[42] S. Dreyer, Mikrostruktur und Transporteigenschaften von Ni/NiO Vielfachschichten, Diplomarbeit, Institut für Materialphysik, Universität Göttingen, (2001)

[43] P. Troche, Thermisch induzierte Gestaltinstabilität von Vielfachschichten, Dissertation, Institut für Materialphysik, Universität Göttingen, (2000)

[44] C. Wagner, Z. physik. Chem. (B), 21,25(1933)

[45] P.Haasen, Physikalische Metallkunde, Springer Verlag Berlin Heidelberg New York Tokyo (1984)

[46] R. Lalauze, M. Soustelle, M. Rekas, J.C. Le Thiesse, Oxidation of Metals, Vol.20, Nos. 5/6, (1983)

[47] E.A.Gulbransen, K.F. Andrew, J. electrochem. Soc. 101, 163, (1954)

[48] Atsushi Takei, Kazuyoshi Nii, Transactions of the Japan Institute of Metals, vol. 17, no. 4, April 1976, p.211-219

[49] Moreau, Benard, Compt. Rend., 237 (1953), 1417

[50] Moreau, Benard, J. Inst. Metals 83 (1954-55), 87

[51] E. Donald, Jr. Larsen, Scripta Metallurgica, Vol. 21, pp 1379-1383, (1987)

[52] F. Czerwinski, J. A. Szpunar, Corrosion Science 41, (1999) ,729-740

[53] W.J. Moore, J.K. Lee, Trans. Faraday Soc. 48, 916, (1952)

[54] A. Atkinson, R.I. Taylor, Philos. Mag A, Vol 43, No 4, 979, (1981)

[55] R. Lindner, Å. Åkerström, Faraday Soc. Disc. 23, (1957) ,133

[57] Hualong Li, F. Czerwinski, J.A. Szunpar, 12 th Int. Conf. on Textures of Mat., S.1391

[58] J.Hoffmann, persönliche Mitteilung, Göttingen, März 2002

[59] O. Kubaschewski, O. von Goldbeck, Z. Metallkunde 39, 158 (1948)

[60] E.A. Gulbransen, K.F. Andrew, J. electrochem. Soc. 101, 128 (1954)

[61] C. Cantoni, D.K. Christen, R. Feenstra, A. Goyal, J.W. Ownby, D.M. Zehner, Applied Physics Letters, vol 79, No 19, (2001) 
[62] F. Czerwinski, J.A. Szpunar, Corrosion Science Vol 39, No 1, 147-158, (1997) 


\section{Danksagung}

Herrn Prof. Dr. H.C. Freyhardt danke ich für die Ermöglichung und die stete Förderung dieser Arbeit.

Ganz besonders möchte ich mich bei Herrn Dr. Jörg Hoffmann für die intensive Betreuung bedanken. Sein Interesse und seine Erfahrungen haben wesentlich zu der Entstehung dieser Arbeit beigetragen.

Bei meiner „Sputtergruppe“: Frau Dr. Sibylle Sievers, Herrn Dr. Jürgen Dzick, Herrn Dr. Peter Troche, Frau Karola Thiele, Herrn Carsten Herweg, Herrn Sebastian Dreyer, Herrn Lars O. Kautschor und Herrn Christian Thiele möchte ich mich für die zahlreichen Diskussionen und die gute Zusammenarbeit bedanken.

Für die Vielzahl an technischer Unterstützung bedanke ich mich bei Herrn Lutz Kirchhof, Frau Katrin Gehrke, Herrn Michael Malchow, Herrn Dieter Plischke und allen Mitarbeitern der feinmechanischen Werkstatt.

Bei Herrn Prof. Dr. W. Kuhs und den Mitarbeitern des Mineralogisch-Kristallographischen Institut der Universität Göttingen bedanke ich mich für die großzügige Bereitstellung von Messzeit am 4-Kreis-Diffraktometer.

Für die REM- und TEM-Aufnahmen der oxidierten NiCr-Filme im Querschnitt bedanke ich mich ganz herzlich bei Herrn Dr. Dominique Eyidi vom Institut für Angewandte Physik der Universität Tübingen.

Herrn Lars O. Kautschor danke ich für die TEM-Aufnahmen, Herrn Carsten Herweg für die AFM-Aufnahmen der NiO-Schichten und Herrn Dr. Christian Jooß für deren Beschichtung mit $\mathrm{YBaCuO}$.

Bei Martin Weisheit und der „Keller-Kaffeerunde“ bedanke ich mich für viele Tips und Diskussionen.

Allen Mitarbeitern des Instituts für Materialphysik und des Zentrums für Funktionswerkstoffe danke ich für ihre Hilfsbereitschaft und für die anregende und angenehme Arbeitsatmosphäre. 


\title{
Lebenslauf
}

\section{Persönliche Daten:}

Name:

Nationalität:

Geburtstag:

Geburtsort:

Eltern:

Cirsten Maja Brandt

deutsch

26.08.1972

Salzgitter-Bad

Heinz-Dieter Brandt und Edith Ahlburg-Brandt geb. Ahlburg

\section{Schulausbildung:}

1979-1983:

1983-1985:

1985-1992

Mai 1992

\author{
Grundschule Othfresen \\ Orientierungstufe Liebenburg \\ Christian-von-Dohm-Gymnasium Goslar \\ Abitur
}

\section{Studium:}

WS 1992

Oktober 1994

WS 1994 - WS 1997

Oktober 1997

SS 1998

Aufnahme des Physikstudiums an der Universität Göttingen Diplomvorprüfung Physik

Hauptstudium Physik

Diplomprüfung Physik

Diplomarbeit bei Herrn Prof. Dr. H.C. Freyhardt am Institut für Metallphysik mit dem Thema:

Remanente Feldverteilung und Levitationskraft von schmelztexturiertem $\mathrm{YBaCuO}$

Beginn der Dissertation in der Arbeitsgruppe von Herrn Prof.

Dr. H.C. Freyhardt am Institut für Materialphysik

\section{Beschäftigungen:}

Seit dem 1.3.1998

Wiss. Mitarbeiterin am Institut für Materialphysik 